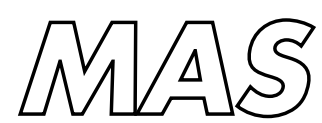

Modelling, Analysis and Simulation 
$\mathrm{CWI}$ is the National Research Institute for Mathematics and Computer Science. It is sponsored by the Netherlands Organization for Scientific Research (NWO).

$\mathrm{CWI}$ is a founding member of ERCIM, the European Research Consortium for Informatics and Mathematics.

CWI's research has a theme-oriented structure and is grouped into four clusters. Listed below are the names of the clusters and in parentheses their acronyms.

Probability, Networks and Algorithms (PNA)

Software Engineering (SEN)

\section{Modelling, Analysis and Simulation (MAS)}

Information Systems (INS)

Copyright (C) 2005, Stichting Centrum voor Wiskunde en Informatica

P.O. Box 94079, 1090 GB Amsterdam (NL)

Kruislaan 413, 1098 SJ Amsterdam (NL)

Telephone +31205929333

Telefax +31205924199

ISSN 1386-3703 


\title{
Stability of spatially periodic pulse patterns in a class of singularly perturbed reaction-diffusion equations
}

\begin{abstract}
In this paper we develop a stability theory for spatially periodic patterns on $\mathbb{R}$. Our approach is valid for a class of singularly perturbed reaction-diffusion equations that can be represented by the generalized Gierer-Meinhardt equations as 'normal form'. These equations exhibit a large variety of spatially periodic patterns. We construct an Evans function $\mathcal{D}(\lambda, \gamma)$ that is defined for the $\gamma$-eigenvalue $\lambda$ in a certain subset of $\mathbb{C}$. The spectrum associated to the stability of the periodic pattern is given by the solutions $\lambda(\gamma)$ of $\mathcal{D}(\lambda(\gamma), \gamma)=0$, where $\gamma \in \mathbf{S}^{1}$. Although our method can be applied to all types of singular pulse patterns, we focus on the stability analysis of the families of most simple periodic solutions. By decomposing $\mathcal{D}(\lambda, \gamma)$ into a product of a 'slow' and a 'fast' Evans function, we are able to determine explicit expressions for the $\gamma$-eigenvalues that are $\varnothing(1)$ with respect to the small parameter $\varepsilon$. Although the branch of 'small' $\gamma$-eigenvalues that is connected to the translational 1-eigenvalue $\lambda(1)=0$ cannot be studied by this decomposition, our methods also enable us determine the location of these $\gamma$-eigenvalues. Thus, our approach provides a full analytical control of the (spectral) stability of the singular spatially periodic patterns. We establish that the destabilization of a periodic pulse pattern on $\mathbb{R}$ is always initiated by the $\mathcal{O}(1) \gamma$-eigenvalues, and consider various kinds of bifurcations. Finally, we apply our insights to the stability problem associated to the restriction of a periodic pulse pattern to a bounded domain with homogeneous Neumann boundary conditions.
\end{abstract}

2000 Mathematics Subject Classification: 35B10, 35B25, 35B32, 35B35, 35K57, 92C15

Keywords and Phrases: reaction-diffusion equations, spatially periodic solutions, 



\title{
Stability of spatially periodic pulse patterns in a class of singularly perturbed reaction-diffusion equations
}

\author{
Harmen van der Ploeg${ }^{1}$, Arjen Doelman ${ }^{2,1}$ \\ ${ }^{1}$ Korteweg-de Vries Instituut, Universiteit van Amsterdam \\ Plantage Muidergracht 24, 1018 TV Amsterdam, the Netherlands \\ ${ }^{2}$ Center for Mathematics and Computer Science (CWI) \\ P.O. Box 94079, 1090 GB Amsterdam, the Netherlands
}

April 25, 2005

\begin{abstract}
In this paper we develop a stability theory for spatially periodic patterns on $\mathbb{R}$. Our approach is valid for a class of singularly perturbed reaction-diffusion equations that can be represented by the generalized Gierer-Meinhardt equations as 'normal form'. These equations exhibit a large variety of spatially periodic patterns. We construct an Evans function $\mathcal{D}(\lambda, \gamma)$ that is defined for the $\gamma$-eigenvalue $\lambda$ in a certain subset of $\mathbb{C}$. The spectrum associated to the stability of the periodic pattern is given by the solutions $\lambda(\gamma)$ of $\mathcal{D}(\lambda(\gamma), \gamma)=0$, where $\gamma \in \mathbf{S}^{1}$. Although our method can be applied to all types of singular pulse patterns, we focus on the stability analysis of the families of most simple periodic solutions. By decomposing $\mathcal{D}(\lambda, \gamma)$ into a product of a 'slow' and a 'fast' Evans function, we are able to determine explicit expressions for the $\gamma$-eigenvalues that are $\varnothing(1)$ with respect to the small parameter $\varepsilon$. Although the branch of 'small' $\gamma$-eigenvalues that is connected to the translational 1-eigenvalue $\lambda(1)=0$ cannot be studied by this decomposition, our methods also enable us determine the location of these $\gamma$-eigenvalues. Thus, our approach provides a full analytical control of the (spectral) stability of the singular spatially periodic patterns. We establish that the destabilization of a periodic pulse pattern on $\mathbb{R}$ is always initiated by the $\mathcal{O}(1) \gamma$-eigenvalues, and consider various kinds of bifurcations. Finally, we apply our insights to the stability problem associated to the restriction of a periodic pulse pattern to a bounded domain with homogeneous Neumann boundary conditions.
\end{abstract}

\section{Introduction}

In recent years, the theory of the stability of localized solutions of homoclinic or heteroclinic type, such as traveling waves, has developed significantly. Moreover, a unifying approach that can be applied to various classes of nonlinear partial differential equations, such as (nearly)integrable systems, reaction-diffusion equations and conservation laws, has become available in the form of the Evans function $\mathcal{D}(\lambda)$, where $\lambda \in \mathbb{C}$ corresponds to a possible eigenvalue of the linearized stability problem associated to the localized 'pattern' (see $[1,12,26,16,19,3,2,21]$ and the references therein). The solitary homoclinic/heteroclinic patterns can be seen as 'building blocks' of more complex patterns. Arguably the most simple of such patterns are the spatially periodic patterns. Based on the insights obtained for the homoclinic/heteroclinic solutions, a theory has been developed by which the spectral stability of these patterns with respect to bounded, in general non-localized, perturbations can be studied. Again, a unifying 
role is played by an Evans function, that is an extension of the 'homoclinic Evans function' $\mathcal{D}(\lambda)$, to a 'spatially periodic Evans function' $\mathcal{D}(\lambda, \gamma)$, in which $\gamma \in \mathbf{S}^{1} \subset \mathbb{C}$ refers to the concept of the $\gamma$-eigenvalue $[10,11]$. As for the homoclinic/heteroclinic case, this approach can again be applied to various classes of equations (see [10,11, 8, 27, 25] and the references therein).

In this paper, a spatially periodic Evans function $\mathcal{D}(\lambda, \gamma)$ is defined and analyzed in the context of the (spectral) stability problem for several types of spatially periodic pulse solutions in a class of singularly perturbed reaction-diffusion equations. In its most general setting, this class of reaction-diffusion equations is a two-component system of the form

$$
\left\{\begin{array}{l}
U_{t}=d_{U} U_{x x}+a_{1} U+a_{2} V+F(U, V) \\
V_{t}=d_{V} V_{x x}+b_{1} U+b_{2} V+G(U, V)
\end{array}\right.
$$

with $x \in \mathbb{R}$ and $0<d_{V} \ll d_{U}$, so that a small parameter $\varepsilon$ can be defined by $\varepsilon^{2}=d_{V} / d_{U} \ll 1$. System (1.1) is assumed to have a stable trivial state $(U(x, t), V(x, t)) \equiv(0,0)$. Provided that a number of (generic) conditions on the parameters $a_{1,2}, b_{1,2} \in \mathbb{R}$ and the (smooth) functions $F, G: \mathbb{R}^{2} \rightarrow \mathbb{R}$ are satisfied (see [3]), these equations exhibit countably many families of various kinds of singular homoclinic and spatially periodic pulse patterns [3, 6]. Here, 'singular' refers to the fact that the amplitudes of the (localized) pulses are in general not $\mathcal{O}(1)$ with respect to the asymptotic parameter $\varepsilon$ (Remark 1.6). Therefore, it is natural to perform a scaling analysis in (1.1), so that the amplitudes of the pulses become $\mathcal{O}(1)$. This is a relatively straightforward procedure that leads, at leading order in $\varepsilon$, to the following 'normal form' [3],

$$
\left\{\begin{array}{rrrrr}
\varepsilon^{2} U_{t} & = & U_{x x} & -\varepsilon^{2} \mu U & +U^{\alpha_{1}} V^{\beta_{1}} \\
V_{t} & =\varepsilon^{2} V_{x x} & -V & +U^{\alpha_{2}} V^{\beta_{2}}
\end{array}\right.
$$

in which $\mu, \alpha_{1,2}, \beta_{1,2} \in \mathbb{R}$ are parameters such that

$$
\mu>0, D=\left(\alpha_{1}-1\right)\left(\beta_{2}-1\right)-\alpha_{2} \beta_{1}>0, \alpha_{2}<0, \beta_{1,2}>1
$$

Note that the nonlinear terms in (1.2) are determined by the leading order behavior of the $F(U, V)$ and $G(U, V)$ in $(1.1)$ - see [3]. In order to minimize the technical details as much as possible, we do not consider the general system (1.1) in this paper, but study the stability of spatially periodic pulse solutions in the normal form reduction (1.2). This is not a restriction. The theory developed here can be applied to the general setting of (1.1), of course under conditions on the parameters $a_{1,2}, b_{1,2}$ and the nonlinearities $F(U, V), G(U, V)$ as formulated in [3]. In fact, the conditions on (1.1) in [3] are even stronger than necessary, see Remark 1.3. Moreover, the leading order corrections to (1.2) that appear from the scaling analysis in (1.1) do not have a leading order influence on the analysis (as has been explicitly shown in [3] for the stability theory of homoclinic patterns).

Apart from the present interpretation as normal form reduction, equation (1.2) also appears in the literature as the 'generalized Gierer-Meinhardt equation', i. e. a generalization of the 'classical' Gierer-Meinhardt equation originally proposed by Gierer and Meinhardt as a model for biological pattern formation or 'morphogenesis' (see [14, 24, 17, 28, 29] and the references therein). At several places in the paper, we will consider the special 'classical' case as an explicit example, i.e. we will set

$$
\alpha_{1}=0, \beta_{1}=2, \alpha_{2}=-1, \beta_{2}=2, \text { and } D=1
$$

(1.3). The generalized Gierer-Meinhardt has a remarkable multitude of (families of) spatially periodic patterns, ranging from simple patterns that consist of identical pulses, to periodic extensions of 'random' (but finite) arrays of several types of pulses [6]. Again, we do not consider the most general setting here, i.e. we do not consider the stability problem for a 'general' spatially periodic pattern. Instead, we only consider the most simple types of patterns. The fundamental spatially periodic patterns $\left(U_{p}(x ; L), V_{p}(x ; L)\right)$ consist, as graphs of $x \in \mathbb{R}$, of identical pulses at a distance $2 L$ apart, see Figure $1(\mathrm{a})$. We distinguish two types of fundamental pulse patterns, the patterns of A-type, $\left(U_{p, A}\left(x ; L_{A}\right), V_{p, A}\left(x ; L_{A}\right)\right)$, and those of B-type 
(a)

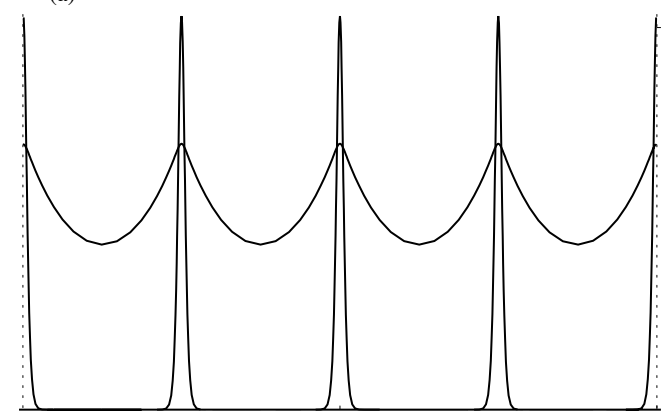

(b)

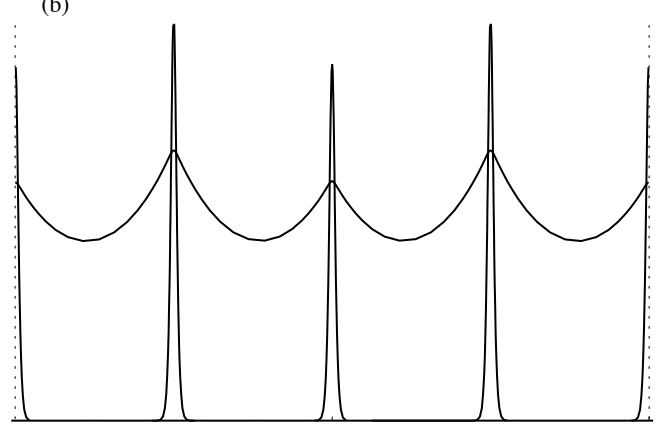

Figure 1: Two examples of spatially periodic pulse solutions of the classical Gierer-Meinhardt equations ((1.2) with (1.4)), restricted to a bounded interval; (a) a fundamental periodic solution $\left(U_{p, A}(x), V_{p, A}(x)\right)$ of A-type, and (b) an AB-pattern $\left(U_{p, A B}(x), V_{p, A B}(x)\right)$. Note that the $V$-components of the solutions are pulse-like, and strongly localized, while the $U$-components vary slowly.

$\left(U_{p, B}\left(x ; L_{B}\right), V_{p, B}\left(x ; L_{B}\right)\right), L_{B}<L_{A}$, that merge in a saddle-node bifurcation of periodic solutions (Theorem 1.7). As a first example of a more complex pattern, we also consider the AB-patterns $\left(U_{p, A B}(x ; L), V_{p, A B}(x ; L)\right)$, that consist of alternating pulses of A- and B-type (Theorem 1.8) - see Section 1.1 and Figure 1(b). Again, our methods can be extended (in a straightforward fashion) to include more complex patterns, as the periodically extended 'random' arrays of A- and B-pulses (see also Remark 1.5). Such an extension is mostly a technical exercise, and we will therefore refrain from presenting the details.

Due to the singularly perturbed nature of (1.2), the character of the spatially periodic patterns depends strongly on the properties of the fast reduced limit problem

$$
\left\{\begin{aligned}
\bar{U} & \equiv U_{0} \\
\bar{V}_{t} & =\bar{V}_{\xi \xi}-\bar{V}+U_{0}^{\alpha_{2}} \bar{V}^{\beta_{2}},
\end{aligned}\right.
$$

which is obtained from (1.2) by introducing the fast spatial variable $\xi=x / \varepsilon$ and taking the limit $\varepsilon \rightarrow 0$ (under the assumption that $U(\xi, t)$ is bounded on $\mathbb{R}$ ). This is a scalar equation for the limit function $\bar{V}(\xi, t) ; U_{0} \in \mathbb{R}$ is the leading order value of the $U$-component of the periodic pattern in the (narrow) $\xi$-region around the $V$-pulse (Figure 1 ), $U_{0}=U_{0}(L)$ depends on the period $(=2 L)$ of the periodic pattern ([6], Section 1.1). The $V$-component of the periodic pulse solutions is approximated by the homoclinic solution $\bar{V}_{h}(\xi ; L)$ of the stationary problem associated to $(1.5)$ - recall that $\beta_{2}>1(1.3)$. Both $U_{0}(L)$ and $\bar{V}_{h}(\xi ; L)$ have welldefined limits as $L \rightarrow \infty$, these are the leading order approximations of the homoclinic pulse $\left(U_{h}(\xi), V_{h}(\xi)\right)=\left(U_{p}(\xi ; \infty), V_{p}(\xi ; \infty)\right)$ that is the natural boundary of the family of $2 L$-periodic solutions $\left(U_{p, A}(\xi ; L), V_{p, A}(\xi ; L)\right)$ (Theorem 1.7$)$. The patterns we study in this paper, have an asymptotically long period: $\varepsilon^{2} L=\mathcal{O}(1)$ (or larger) in the $\xi$-coordinate (Theorems 1.7 and 1.8). Nevertheless, this length scale corresponds to the natural length scale of the $U$-components in the regions between two pulses $(\mathcal{O}(1 / \varepsilon)$ in the slow $x$-scaling, see (1.14)). Hence, for $L$ such that $\varepsilon^{2} L=\mathcal{O}(1)$, subsequent pulses of a periodic pattern are so 'close' to each other that the $U$-components remain $\mathcal{O}(1)$ in between the pulses. Thus, the $U$-component of a periodic pattern does not (necessarily) become exponentially small in between the pulses. Also, $U_{0}(L)$ and $\bar{V}_{h}(\xi ; L)$ are not necessarily close to $U_{0}(\infty)$ and $\bar{V}_{h}(\xi ; \infty)$, i.e. the pattern $\left(U_{p}(\xi ; L), V_{p}(\xi ; L)\right)$ is not necessarily close to its homoclinic limit $\left(U_{p}(\xi ; \infty), V_{p}(\xi ; \infty)\right)$ (restricted to one period $[0,2 L])$.

Unlike in [11] and [27], we can therefore not assume that the period $2 L$ of $\left(U_{p}(\xi ; L), V_{p}(\xi ; L)\right)$ is 'large enough'. As a consequence, the theory presented here extends that of [11,27], which is developed for near-homoclinic patterns in which all components of the periodic pattern are exponentially small between subsequent pulses. On the other hand, the results of [11, 27] are, of 
course, valid in a much larger class of (not necessarily singularly perturbed) reaction-diffusion equations than (1.1) and (1.2) considered here. Moreover, our results can (and will) be checked against $[11,27]$ by considering the limit $\varepsilon^{2} L \rightarrow \infty$.

Independent of the type of the $2 L$-periodic pattern $\left(U_{p}(\xi ; L), V_{p}(\xi ; L)\right)$, the linearized stability on $\mathbb{R}$ of the pattern with respect to bounded and uniformly continuous perturbations is determined by a linear equation of the form

$$
\frac{d}{d \xi} \phi=A_{p}(\xi ; \lambda, L, \varepsilon) \phi
$$

in which $\lambda \in \mathbb{C}$ is the eigenvalue that determines the linear growth of a perturbation, $\phi: \mathbb{R} \rightarrow \mathbb{C}^{4}$ its potential eigenfunction, and $A_{p}(\xi)$ a $4 \times 4$ matrix that is $2 L$-periodic (see Section 2 for the details). Floquet theory implies that $\lambda \in \mathbb{C}$ is an eigenvalue of (1.6) if and only if there is a $\gamma \in \mathbf{S}^{1}=\{\gamma \in \mathbb{C}:|\gamma|=1\}$ and a nontrivial $\phi(\xi)$ such that $\phi(\xi+2 L)=\gamma \phi(\xi)$ for all $\xi \in \mathbb{R}$. These $\lambda=\lambda(\gamma)$ 's are the $\gamma$-eigenvalues, and the full spectrum of (1.6) consists of a number of curves of these $\gamma$-eigenvalues, parameterized by $\gamma \in \mathbf{S}^{1}$ ([10], Definition 2.1, Proposition 2.2).

In essence, the Evans function $\mathcal{D}(\lambda, \gamma ; L)$ for the stability problem (1.6) is a determinant that determines whether a monodromy matrix associated to a fundamental (matrix) solution $\Psi(\xi ; \lambda)$ of (1.6) has eigenvalues $\gamma \in \mathbf{S}^{1}$ (Section 2.1). As a consequence, $\lambda(\gamma)$ is a $\gamma$-eigenvalue if and only if $\mathcal{D}(\lambda(\gamma), \gamma ; L)=0$, counting multiplicities ([10], Section 2.1). Following the approach of $[3,4]$, an especially suitable set of independent solutions to $(1.6)$, i.e. a matrix $\Psi(\xi ; \lambda)$, can be determined that facilitates the construction of $\mathcal{D}(\lambda, \gamma ; L)$ (Section 3). As is usual for an Evans function, this construction breaks down (or has to be adapted $[13,20]$ ) if $\lambda$ is close to the spectrum associated to the trivial background state, i.e. the solution $(U(x, t), V(x, t)) \equiv(0,0)$ of (1.2). Given that $\mu>0$, this spectral set is part of the stable half plane and hence it has no influence on the stability of $\left(U_{p}(\xi ; L), V_{p}(\xi ; L)\right)$, so that cutting out a small neighborhood of this set from the complex plane does not influence the stability analysis. Therefore, we introduce a second (artificial) small parameter $\delta$ so that $0<\varepsilon \ll \delta \ll 1$ (Remark 1.6) and define

$$
\mathbb{C}_{e}=\mathbb{C} \backslash\{\lambda \in \mathbb{C} \mid \operatorname{Re}[\lambda]<\max (-1,-\mu)+\delta \text { and }|\operatorname{Im}[\lambda]|<\delta\},
$$

(Remark 1.4). The eigenvalues $\lambda_{j}^{r}$ of the eigenvalue problem associated to the stability of the homoclinic solution $\bar{V}_{h}(\xi ; L)$ of $(1.5)$ play a crucial role in the stability analysis of the periodic patterns. These $\lambda_{j}^{r}$ 's do not depend on $L, \lambda_{j}^{r}=\lambda_{j}^{r}\left(\beta_{2}\right)$ with $\lambda_{0}^{r}=\frac{1}{4}\left(\beta_{2}+1\right)^{2}-1>0, \lambda_{1}^{r}=0$, and $\lambda_{j}^{r}<0$ for $j=2,3, \ldots, J-1$ with $J=J\left(\beta_{2}\right) \geq 2$, see [3] and Lemma 3.3. In fact, in the construction of $\mathcal{D}(\lambda, \gamma ; L)$ it is necessary to distinguish between the cases in which $\lambda$ is close, and in which $\lambda$ is not close to a $\lambda_{j}^{r}$. Hence, we define $\mathbb{C}_{r}$ by

$$
\mathbb{C}_{r}=\mathbb{C}_{e} \backslash \bigcup_{j=0, \ldots, J-1} B\left(\lambda_{j}^{r}, \delta\right),
$$

with $\delta$ as in the definition of $\mathbb{C}_{e}$, and $B\left(\lambda_{j}^{r}, \delta\right)$ a small ball around $\lambda_{j}^{r}$, i.e.

$$
B\left(\lambda_{j}^{r}, \delta\right)=\left\{\lambda \in \mathbb{C}:\left|\lambda-\lambda_{j}^{r}\right|<\delta\right\} .
$$

The following theorem summarizes the theory developed in Sections 3, 4 and 5.

Theorem 1.1 Assume that (1.3) holds, let $0<\varepsilon \ll \delta \ll 1$ and let $\mathcal{D}(\lambda, \gamma, L)$ be the Evans function associated to the stability problem (1.6) of a fundamental spatially periodic pattern $\left(U_{p}(\xi ; L), V_{p}(\xi ; L)\right)$ with period $2 L$ (Theorem 1.7).

(I) $\lambda \in \mathbb{C}_{r}$

For $\lambda \in \mathbb{C}_{r}$ and $\gamma \in \mathbf{S}^{1}, \mathcal{D}(\lambda, \gamma ; L)$ can be decomposed into a product of a fast component, $\mathcal{D}^{f}(\lambda, \gamma ; L)$, and a slow component, $\mathcal{D}^{s}(\lambda, \gamma ; L) ; \mathcal{D}^{f}(\lambda, \gamma ; L) \neq 0$ for all $\lambda \in \mathbb{C}_{r}$, so 
that all $\gamma$-eigenvalues $\lambda=\lambda(\gamma, \varepsilon) \in \mathbb{C}_{r}$ of (1.6) must be zeroes of $\mathcal{D}^{s}(\lambda, \gamma ; L)$. Moreover, $\lambda=\lambda(\gamma, \varepsilon)=\lambda(\gamma, 0)+\mathcal{O}(\varepsilon)$ solves $\mathcal{D}^{s}(\lambda, \gamma ; L)=0$ if and only if $\lambda(\gamma, 0)$ satisfies

$$
T^{s}(\lambda, L) \stackrel{\text { def }}{=} \frac{1+\Delta^{4}}{\Delta^{2}}-\frac{\left(1-\Delta^{4}\right) \sqrt{\mu}}{\Delta^{2} \sqrt{\mu+\lambda}} \tanh \left(\varepsilon^{2} \sqrt{\mu} L\right)\left[\alpha_{1}-\alpha_{2} \beta_{1} \mathcal{R}(\lambda)\right] \in[-2,2],
$$

where

$$
\Delta=\Delta(\lambda, L)=e^{-\varepsilon^{2} \sqrt{\mu+\lambda} L} \in \mathbb{C}, \text { with }|\Delta| \in(0,1),
$$

$\left(\arg [\sqrt{\mu+\lambda}] \in\left(-\frac{\pi}{2}, \frac{\pi}{2}\right)(1.7)\right) ; \mathcal{R}(\lambda)$ is an explicitly known expression (4.12) that also appears in the stability analysis of homoclinic patterns [3]. The value of $\gamma \in \mathbf{S}^{1}$ (and that of $\bar{\gamma} \in \mathbf{S}^{1}$, Proposition 2.2) is determined by $T^{s}(\lambda, L)=2 \operatorname{Re}[\gamma]$.

(II) $\lambda \in B\left(\lambda_{j}^{r}, \delta\right)$

Assume that also $\left|\alpha_{2}\right| \gg \sqrt{\delta},\left|\alpha_{1}\right|=\mathcal{O}(1)$, and $\varepsilon^{2} L=\mathcal{O}(1)$ or $\varepsilon^{2} L \gg 1$ with respect to $\delta$.

- If $j=2 k$ is even, then eigenvalue problem (1.6) has no $\gamma$-eigenvalues in the ball $B\left(\lambda_{2 k}^{r}, \delta\right)$.

- If $j=2 k+1$ is odd and if $T^{s}\left(\lambda_{2 k+1}^{r}, L\right) \notin[-2,2]$, i.e. if there is no $\mathcal{O}(1) \gamma$-eigenvalue described by (1.10) near $\lambda=\lambda_{2 k+1}^{r}$, then eigenvalue problem (1.6) has one (and only one) $\gamma$-eigenvalue $\lambda(\gamma) \in B\left(\lambda_{2 k+1}^{r}, \delta\right)$ for every $\gamma \in \mathbf{S}^{1}$ (counting multiplicities); if $T^{s}\left(\lambda_{2 k+1}^{r}, L\right) \in[-2,2]$ then (1.6) has one $\gamma$-eigenvalue $\lambda(\gamma) \in B\left(\lambda_{2 k+1}^{r}, \delta\right)$ for any $\gamma \in \mathbf{S}^{1}$ with $\left|\operatorname{Re}[\gamma]-\frac{1}{2} T^{s}\left(\lambda_{2 k+1}^{r}, L\right)\right| \gg \delta$.

(III) $\lambda \in B(0, \delta)$

All small $\gamma$-eigenvalues in $B\left(\lambda_{1}^{r}=0, \delta\right)$ are $\mathcal{O}\left(\varepsilon^{4}\right)$.

- If $T^{s}(0, L) \notin[-2,2]$, i.e. if there is no $\mathcal{O}(1) \gamma$-eigenvalue near $\lambda=0$, then $\lambda(\gamma) \in[\lambda(-1), \lambda(1)]$ for all $\gamma \in \mathbf{S}^{1}$, with $\lambda(-1)<0=\lambda(1)$; the multiplicity of $\lambda(1)=0$, i.e. the eigenvalue associated translations of $\left(U_{p}(\xi ; L), V_{p}(\xi ; L)\right)$, is 1 .

- If $T^{s}(0, L) \in(-2,2)$, then $\lambda(\gamma) \in \mathbb{R} \backslash(\lambda(-1), \lambda(1))$ for all $\gamma \in \mathbf{S}^{1}$ with $\left|\operatorname{Re}[\gamma]-\frac{1}{2} T^{s}(0, L)\right| \gg \delta$; $\lambda(1)=0$ is simple.

This theorem is a combination of Proposition 3.8 and the calculations of Section 4.2 (part (I)), Proposition 4.3 (II), and Lemmas 5.2 and 5.3 (III). The additional conditions in (II) are not relevant to the stability question, since $\left(U_{p}(\xi ; L), V_{p}(\xi ; L)\right)$ is unstable if any of these conditions are violated (Remarks 4.4 and 4.5). Based on Propositions 7.2, 7.3 and Section 7.2, a similar theorem could be formulated for the stability problem associated to the periodic patterns $\left(U_{p, A B}(\xi ; L), V_{p, A B}(\xi ; L)\right)$ of AB-type. The main difference between these two cases is that explicit expression (1.10) becomes more complicated in the AB-case (see (7.6) in Proposition 7.2 ), and that the number of $\gamma$-eigenvalues near the reduced eigenvalues $\lambda_{2 k+1}^{r}$ is 2 instead of 1 (per allowed $\gamma \in \mathbf{S}^{1}$, Proposition 7.3). The analysis is very similar (Section 7). In fact, it is clear from the analysis of the AB-patterns, that the same approach can be applied to each of the more complex periodic patterns constructed in [6] (see also Remark 1.5). Again, the main difference will be the complexity of the equivalents of (1.10) and (7.6), and the number of $\gamma$-eigenvalues near a $\lambda_{2 k+1}^{r}$. As an explicit example, we have considered the stability of a $A A B B$-pattern in Remark 7.4.

The methods by which the results presented in Theorem 1.1 have been obtained are based on the approach developed in $[3,4]$. It is found that many of the building blocks of the theory that led to the decomposition of the homoclinic Evans function $\mathcal{D}(\lambda ; \varepsilon)$ have a natural counterpart in that of the theory for the periodic Evans function $\mathcal{D}(\lambda, \gamma ; L, \varepsilon)$ developed here. Of course there are some differences. For instance, the homoclinic limit does not have small $\mathcal{O}\left(\varepsilon^{4}\right)$ eigenvalues (this interval described in Theorem 1.1 (III) shrinks to the point $\lambda=0$ in the limit $L \rightarrow \infty)$.

A more remarkable difference is that the pole-zero cancellation of the homoclinic limit, also 
called 'the NLEP paradox' in [3, 4], has become a much more subtle phenomenon. In the homoclinic case, $\mathcal{D}(\lambda)$ decomposes into the product $\mathcal{D}^{f}(\lambda) \mathcal{D}^{s}(\lambda)$ for all $\lambda \in \mathbb{C}_{e}$. The fast component $\mathcal{D}^{f}(\lambda)$ is analytic and has zeroes at $\lambda_{j}^{*}$, where $\lambda_{j}^{*}$ is asymptotically close to the eigenvalues $\lambda_{j}^{r}$ associated to the stability of the homoclinic solution $\bar{V}_{h}(\xi ; \infty)$ of the fast reduced limit problem (1.5). The slow component $\mathcal{D}^{s}(\lambda)$ has simple poles at the zeroes $\lambda_{j}^{*}$ of $\mathcal{D}^{f}(\lambda)$ for $j$ even. Thus, the unstable zero $\lambda_{0}^{*}>0$ of $\mathcal{D}^{f}(\lambda)$ is removed by this cancellation mechanism and the homoclinic pulse can be stable (while its fast reduced limit has an $\mathcal{O}(1)$ unstable eigenvalue). In the periodic case, the decomposition of $\mathcal{D}(\lambda, \gamma)$ into the product $\mathcal{D}^{f}(\lambda, \gamma) \mathcal{D}^{s}(\lambda, \gamma)$ breaks down near the fast reduced eigenvalues $\lambda_{j}^{r}$. Outside $B\left(\lambda_{j}^{r}, \delta\right)$, the fast component $\mathcal{D}^{f}(\lambda, \gamma)$ is asymptotically close to the periodic Evans function of nearly-homoclinic periodic solutions of (1.5), that has $\gamma$-eigenvalues near each $\lambda_{j}^{r}$ (Lemma 3.4). However, it is a priori not even clear whether $\mathcal{D}^{f}(\lambda, \gamma)$ can be extended inside $B\left(\lambda_{j}^{r}, \delta\right)$ as a smooth (analytic) function. As a consequence there is no definite information on the (possible) zeroes of $\mathcal{D}^{f}(\lambda, \gamma)$ near $\lambda_{j}^{r}$. Thus it does not necessarily follow that $\mathcal{D}^{s}(\lambda, \gamma)$ has poles - although it is certainly also not clear whether $\mathcal{D}^{s}(\lambda, \gamma)$ is analytic (see Section 4.3). Nevertheless, we can apply an adapted version of the winding number arguments of $[3,4]$ over $\partial B\left(\lambda_{j}^{r}, \delta\right)$ to obtain a result that is very similar to the pole-zero cancellation result of the homoclinic limit (Theorem 1.1 (II) or Proposition 4.3).

Theorem 1.1 contains all information that is necessary to establish the linear stability of $\left(U_{p}(\xi ; L), V_{p}(\xi ; L)\right)$ (see Remark 1.2). Section 6 is devoted to the stability analysis of the fundamental periodic patterns. It is found that all patterns of B-type are unstable (with $\mathcal{O}(1)$ unstable $\gamma$-eigenvalues (Corollary 6.6)). An A-pattern $\left(U_{p, A}(\xi ; L),\left(V_{p, A}(\xi ; L)\right)\right.$ with $\varepsilon^{2} L=\varepsilon^{2} L_{A} \gg 1$ is stable, if it is near a homoclinic limit that is stable (Corollary 6.2). As $L$ decreases, $\left(U_{p, A}(\xi ; L),\left(V_{p, A}(\xi ; L)\right)\right.$ may destabilize by a Hopf bifurcation, or by the saddle-node bifurcation, as it merges with a B-pattern (Theorems 6.8 and 6.9). In Section 7.3, a similar analysis is performed for the patterns of AB-type. There, it is established that the AB-patterns are unstable with respect to $\mathcal{O}(1)$ eigenvalues (Corollary 7.6).

Furthermore, our explicit methods are considered in the limit $L \gg 1$ in somewhat more detail. If $\lambda_{h} \in \mathbb{C} \backslash \mathbb{R}$ is an eigenvalue of the stability problem associated to the homoclinic limit $\left(U_{h}(\xi), V_{h}(\xi)\right)=\left(U_{p}(\xi ; \infty), V_{p}(\xi ; \infty)\right)$ (Theorem 1.7), then it follows from [11] that there is a curve of $\gamma$-eigenvalues $\left\{\lambda=\lambda(\gamma), \gamma \in \mathbf{S}^{1}\right\}$ that approaches $\lambda_{h}$ as $L \rightarrow \infty$. Here, it is shown that this curve is at leading order a straight line between the 'endpoints' $\lambda(-1)$ and $\lambda(+1)$, that shrinks, and rotates around its center $\lambda( \pm i)$ as $L$ increases (Lemma 6.1). Although the B-pulse shrinks to 0 , and the A-pulse converges to the homoclinic pulse in the limit $L \rightarrow \infty$, the results of $[11,27]$ cannot be applied to the AB-patterns. In fact, we show that the AB-patterns have spectrum near the eigenvalues of the homoclinic limit for $L \gg 1$, which agrees with [11], but that there also is a small curve of $\gamma$-eigenvalues near every even eigenvalue $\lambda_{2 k}^{r}$ associated to the reduced problem (1.5) - Lemma 7.7.

Finally, in Section 8 we consider the restriction of the stability problem posed on $\mathbb{R}$ to a bounded interval $[0, X]$ with homogeneous Neumann boundary conditions, and compare our results to $[17,28,29]$. It follows from [6] that a fundamental pattern $\left(U_{p}(\xi ; L),\left(V_{p}(\xi ; L)\right)\right.$ restricted to $[0, X]$ can be of three types, depending on the number of (half-)pulses on the boundaries - see also Figure 10. The stability characteristics of a pulse pattern restricted to $[0, X]$ strongly depends on this type. For instance, we show that the saddle-node bifurcation at which an A-pulses transforms into a B-pulse generates $\mathcal{O}(1)$ unstable eigenvalues (Corollary 6.6). This seems to be in contradiction with the results of $[17,28]$, where it is shown that this destabilization is driven by the small $\mathcal{O}\left(\varepsilon^{4}\right)$ eigenvalues. However, if the patterns have no (half-)pulses on the boundaries, i.e. if the pattern is of the type considered by [17, 28], then the most unstable $\mathcal{O}(1) \gamma=-1$-eigenfunction cannot satisfy the boundary conditions (Corollary 8.3), and it indeed also follows from our methods that this bounded pattern is destabilized by the (less unstable) $\mathcal{O}\left(\varepsilon^{4}\right)$-eigenvalues (Corollary 8.4). The -1-eigenfunction is a solution of the finite interval problem if the pattern has half-pulses at both boundaries (as in Figure 1). In this case, it is destabilized by the $\mathcal{O}(1)$ eigenvalue (Corollary 8.4). We have confirmed these 
somewhat subtle distinctions by numerical simulations (see Figure 10).

Remark 1.2 It follows from Theorem 1.1 that a periodic pattern $\left(U_{p}(\xi ; L), V_{p}(\xi ; L)\right)$ is linearly stable if all $\mathcal{O}(1)$ eigenvalues are in the complex left half plane, since the $\mathcal{O}\left(\varepsilon^{4}\right)$ eigenvalues are negative, and the translational eigenvalue at $\lambda=0$ is simple. Nevertheless, one can of course not conclude that $\left(U_{p}(\xi ; L), V_{p}(\xi ; L)\right)$ is stable in a nonlinear sense, since the curve of $\mathcal{O}\left(\varepsilon^{4}\right)$ eigenvalues is connected to $\lambda=0$. In this paper we do not consider the nonlinear stability of periodic solutions of $(1.2)$ on $\mathbb{R}$. In fact, this issue has not even been settled for the homoclinic limit patterns (see Remark 1.3 in [3]), although it should be remarked that the homoclinic limit problem involves additional problems that do not appear in the periodic setting $\left(U_{p}(\xi ; L)\right.$ remains bounded away from 0 for $L<\infty$, while $U_{h}(\xi)=U_{p}(\xi ; \infty) \rightarrow 0$ as $\xi \rightarrow \pm \infty$; this causes problems in (1.2) since $\alpha_{2}<0(1.3)$ ). The spectrum of the linear problem associated to a periodic pulse pattern restricted to a bounded interval $[0, X]$ with homogeneous Neumann boundary conditions consists of discrete eigenvalues, and is a subset of the spectrum associated to that periodic pattern as solution of $(1.2)$ on $\mathbb{R}$ (Section 8 ). The translational eigenvalue $\lambda(1)=0$ is not an element of this subset, since its 1-eigenfunction, i.e. the $\xi$-derivative of $\left(U_{p}(\xi ; L), V_{p}(\xi ; L)\right)$, is odd with respect to the boundaries $\xi=0$ and $\xi=X$. Thus, if all $\mathcal{O}(1)$ eigenvalues are in the stable half plane, the standard results as for instance given in [15] can be applied, and it can be concluded that the bounded domain pattern is nonlinearly stable.

Remark 1.3 The Gray-Scott system (see [4] and the references therein) cannot be scaled exactly into the Gierer-Meinhardt normal form (1.2). Nevertheless, it can be scaled into a form that is slightly more general than $(1.2)$ - see [5]. The stability theory for spatially periodic patterns presented in this paper can also be developed for the classes of reaction-diffusion equations considered in [5]. The approach is completely analogous. As a consequence, the methods developed here can also be applied to the Gray-Scott model, the Schnakenberg model, etc. [5].

Remark 1.4 In this paper, the search for eigenvalues is restricted to $\mathbb{C}_{e}$. The set $\mathbb{C} \backslash \mathbb{C}_{e}$ contains the essential spectrum of the stability problem associated to the homoclinic solution $\left(U_{h}(\xi), V_{h}(\xi)\right)=\left(U_{p}(\xi ; \infty), V_{p}(\xi ; \infty)\right)$ (Theorem 1.7, [3]). In the homoclinic limit, the essential, or continuous, spectrum contains eigenfunctions that oscillate in the regions beyond the pulse, i.e. that do not decay to zero as $\xi \rightarrow \pm \infty$. As already noted, the restriction to $\lambda \in \mathbb{C}_{e}$ has no influence on the stability question for $\left(U_{p}(\xi ; L), V_{p}(\xi ; L)\right)$. Nevertheless, the excluded region $\mathbb{C} \backslash \mathbb{C}_{e}$ may contain $\gamma$-eigenvalues of (1.6), for which the $\gamma$-eigenfunctions have oscillatory parts in the regions in between the pulses. See for instance [23], from which it follows for the special case of the Hill's equation, that the essential spectrum associated to a homoclinic limit indeed breaks up in intervals of $\gamma$-eigenvalues for the nearly-homoclinic periodic solutions. See also Remark 8.6.

Remark 1.5 The A- and B-pulses have $V$-components with precisely one maximum in each pulse region. It is shown in [6] that there also exist many families of periodic solutions to (1.2) that have multiple pulses in a pulse region. Such solutions have 'clusters' of one or more asymptotically close pulses at distances of $\mathcal{O}(\varepsilon|\log \varepsilon|)$ from each other (in the $x$-scale). It has been shown in [3] that the associated homoclinic multi-pulse patterns cannot be stable. Therefore, we do not pay attention to the stability of these patterns in this paper. It should be remarked, however, that our methods also apply to these (unstable) patterns.

Remark 1.6 In this paper, we use the standard notation/terminology of the analysis of (singular) perturbations, such as ' $\ll$ ', ' $\mathcal{O}(\varepsilon)$ ' etc., see for instance [7]. Hence, the fact that a statement $\mathcal{S}_{\varepsilon}$ is valid under the assumption 'Let $0<\varepsilon \ll \delta \ll 1$ ' as in Theorem 1.1 and many other Propositions and Lemmas in this paper, is equivalent to the formulation 'There is an $\delta_{0}>0$, such that for all $\delta<\delta_{0}$ there is an $\varepsilon_{0}=\varepsilon_{0}(\delta)<\delta$ such that for all $\varepsilon<\varepsilon_{0}, \mathcal{S}_{\varepsilon}$ holds'. All statements made in this paper are under the assumptions that $0<\varepsilon \ll 1$ and that (1.3) holds. 


\subsection{Existence of periodic pulse solutions}

A stationary solution to (1.2) is a solution of the ordinary differential equation

$$
\left\{\begin{aligned}
u^{\prime} & =p \\
p^{\prime} & =-u^{\alpha_{1}} v^{\beta_{1}}+\varepsilon^{2} \mu u \\
\varepsilon v^{\prime} & =q \\
\varepsilon q^{\prime} & =v-u^{\alpha_{2}} v^{\beta_{2}}
\end{aligned}\right.
$$

where' denotes the spatial derivative with respect to the slow spatial variable $x$. For $0<\varepsilon \ll 1$, this system exhibits an invariant slow manifold $\mathcal{M}$ defined by

$$
\mathcal{M}=\{v=q=0, u>0\} .
$$

The spatially periodic pulse solutions $\left(U_{p}(x), V_{p}(x)\right)$ of $(1.2)$ we consider in this paper are periodic solutions $\left(u_{p}(x), p_{p}(x), v_{p}(x), q_{p}(x)\right)$ of (1.12) that consist of long slow segments close to $\mathcal{M}$ interspersed by short 'jumps' away from $\mathcal{M}$. We refer to [6] for the details of the construction, that is based on geometric singular perturbation theory (see [18]).

During a slow segment, a solution to (1.12) follows the flow on the slow manifold, which is given by

$$
u^{\prime \prime}=\varepsilon^{2} \mu u .
$$

Note that $\frac{d}{d x}=\mathcal{O}(\varepsilon)$, hence the flow on the slow manifold is actually 'super slow' with a typical length scale of $\mathcal{O}(1 / \varepsilon)$. The ODE (1.14) has a saddle point $S$ in the origin, with one-dimensional stable and unstable manifolds $\ell^{u, s}=\{p= \pm \varepsilon \sqrt{\mu} u\}$. The solutions to (1.14) are given by the solution curves

$$
\Gamma_{\nu}=\left\{p^{2}=\varepsilon^{2}\left(\mu u^{2}-\nu\right)\right\} \text { for } \nu \in \mathbb{R} .
$$

For $\nu \rightarrow 0$, the curve $\Gamma_{\nu}$ approaches the union of $\ell^{u}$ and $\ell^{s}$, while for $\nu<0$ the curves $\Gamma_{\nu}$ cross the $p$-axis and the $u$ coordinate becomes negative. Therefore we focus on $\Gamma_{\nu}$ with $\nu>0$ for the construction of periodic solutions (with positive $u$ ). The solutions on $\Gamma_{\nu}$ can be written as

$$
u\left(x ; \nu ; x_{0}\right)=U_{\min }(\nu) \cosh \left(\varepsilon \sqrt{\mu}\left(x-x_{0}\right)\right),
$$

with minimum

$$
U_{\min }(\nu)=\sqrt{\frac{\nu}{\mu}}>0 .
$$

During the 'jumps' away from $\mathcal{M}$, system (1.12) is written in its fast form

$$
\left\{\begin{array}{l}
\dot{u}=\varepsilon p \\
\dot{p}=-\varepsilon u^{\alpha_{1}} v^{\beta_{1}}+\varepsilon^{3} \mu u \\
\dot{v}=q \\
\dot{q}=v-u^{\alpha_{2}} v^{\beta_{2}}
\end{array}\right.
$$

where ${ }^{\cdot}$ denotes the derivative with respect to $\xi$. By considering $\varepsilon \rightarrow 0$, we obtain the fast reduced limit,

$$
\ddot{v}=v-u^{\alpha_{2}} v^{\beta_{2}}, \quad u \equiv U_{0}, \quad p \equiv P_{0},
$$

where $U_{0}>0, P_{0} \in \mathbb{R}$ are constants. Note that (1.19) is the stationary problem associated to (1.5). This system has a homoclinic orbit $v_{h}^{r}\left(\xi ; U_{0}\right)\left(=\bar{V}_{h}(\xi)\right)$ given by

$$
v_{h}^{r}\left(\xi ; U_{0}\right)=U_{0}^{-\alpha_{2} /\left(\beta_{2}-1\right)} w_{h}(\xi), w_{h}\left(\xi ; \beta_{2}\right)=\left(\frac{\beta_{2}+1}{2}\right)^{\frac{1}{\beta_{2}-1}}\left(\operatorname{sech}\left(\frac{1}{2}\left(\beta_{2}-1\right) \xi\right)\right)^{\frac{2}{\beta_{2}-1}}
$$

Systems (1.12) and (1.18) exhibit the same reversibility symmetry as equation (1.2),

$$
\xi, x \rightarrow-\xi,-x, p \rightarrow-p, q \rightarrow-q
$$




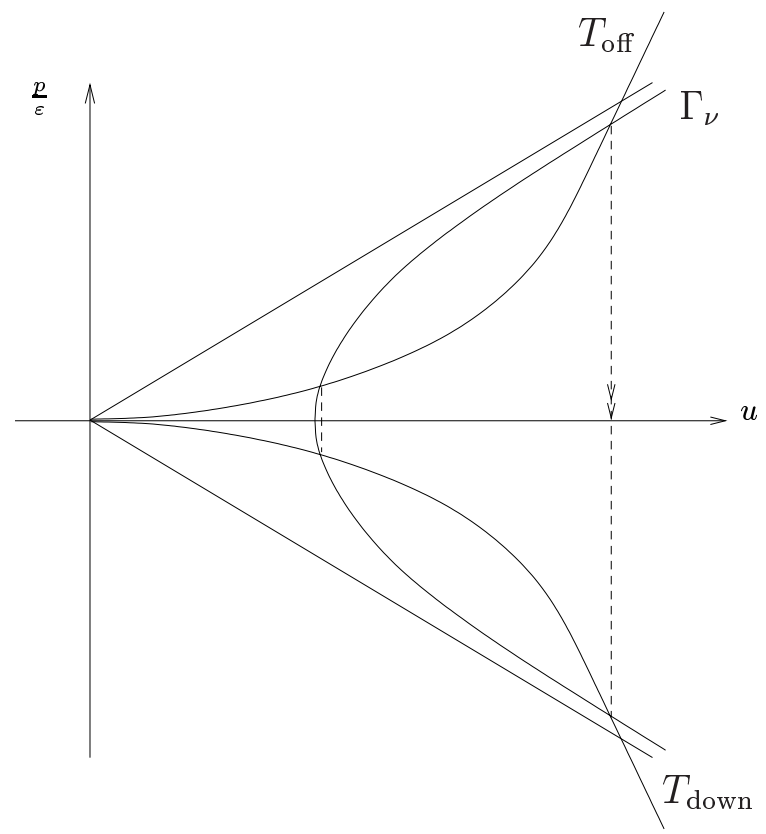

Figure 2: The take off and touch down curves superimposed on the linear flow (1.14) on $\mathcal{M}$.

The reversibility symmetry plays an important role in the construction and the stability analysis of the periodic solutions.

The slow manifold $\mathcal{M}$ is normally hyperbolic and has (3-dimensional, smooth) stable and unstable manifolds $W^{u}(\mathcal{M})$ and $W^{s}(\mathcal{M})$ [9, 18]. The intersection $W^{u}(\mathcal{M}) \cap W^{s}(\mathcal{M})$ consists of countably many 2-dimensional manifolds $[3,6]$. Here, we only consider the manifold spanned by solutions to (1.18) homoclinic to $\mathcal{M}$ that only make one circuit through the fast space (close to a reduced homoclinic orbit $v_{h}^{r}\left(\xi ; U_{0}\right)(1.20)$ ). With a slight abuse of notation we identify $W^{u}(\mathcal{M}) \cap W^{s}(\mathcal{M})$ with this branch - the other manifolds correspond to (unstable) multi-pulse orbits (Remark 1.5). The one-parameter family of homoclinic orbits $\left(u_{\mathcal{M}}(\xi), p_{\mathcal{M}}(\xi), v_{\mathcal{M}}(\xi), q_{\mathcal{M}}(\xi)\right)$ can be parameterized by the base points of the Fenichel fibers [9] on $\mathcal{M}$ of $W^{u}(\mathcal{M}) \cap W^{s}(\mathcal{M})$ as subset of $W^{u}(\mathcal{M})$, i.e. the take off curve $T_{\text {off }} \subset \mathcal{M}$, and of $W^{u}(\mathcal{M}) \cap W^{s}(\mathcal{M})$ as subset of $W^{s}(\mathcal{M})$, i.e. the touch down curve $T_{\text {down }} \subset \mathcal{M}[3,6]$. These curves have been determined explicitly in $[3,6]$,

$$
\begin{array}{ll}
T_{\text {off }} & :\left\{p=p_{d}(u)=\frac{1}{2} \varepsilon u^{1+\frac{D}{\beta_{2}-1}} W\left(\beta_{1}, \beta_{2}\right)+\mathcal{O}\left(\varepsilon^{2}\right)\right\} \\
T_{\text {down }} & :\left\{p=-p_{d}(u)\right\}
\end{array}
$$

where

$$
W\left(\beta_{1}, \beta_{2}\right)=\int_{-\infty}^{\infty}\left(w_{h}\left(\xi ; \beta_{2}\right)\right)^{\beta_{1}} d \xi
$$

$(1.20)$ - see Figure 2. Since $D /\left(\beta_{2}-1\right)>0(1.3)$, both $T_{\text {off,down }}$ are tangent to the $u$-axis, they intersect the stable and unstable manifolds $\ell^{u, s}$ of the saddle $S \in \overline{\mathcal{M}}$, i.e. $\Gamma_{0}(1.15)$, in two symmetric points $(\neq S)$ that have the $u$-coordinate,

$$
U_{\text {hom }}=U_{\text {hom }}\left(\alpha_{1}, \alpha_{2}, \beta_{1}, \beta_{2}, \mu ; \varepsilon\right)=\left(\frac{2 \sqrt{\mu}}{W\left(\beta_{1}, \beta_{2}\right)}\right)^{\frac{\beta_{2}-1}{D}}+\mathcal{O}(\varepsilon) .
$$

By construction, these two points represent the (one-pulse) homoclinic solution $\left(U_{h}(\xi), V_{h}(\xi)\right)$ to (1.2), i.e. $U_{h}(\xi), V_{h}(\xi) \rightarrow 0$ as $\xi \rightarrow \pm \infty ; U_{h}(\xi), V_{h}(\xi)$ are the $u$-, $v$-components of the solution $\gamma_{h}(\xi)=\left(u_{h}(\xi), p_{h}(\xi), v_{h}(\xi), q_{h}(\xi)\right)$ of $(1.18)$ that is homoclinic to $S$. As function of an increasing $\xi, \gamma_{h}(\xi)$ is first exponentially close to $\ell^{u} \subset \mathcal{M}$, then 'takes off' from $\mathcal{M}$ near $\ell^{u} \cap T_{\text {off }}$, makes 
a circuit through the fast $(v, q)$-space $\mathcal{O}(\varepsilon)$ close to $\left(U_{\mathrm{hom}}, 0, v_{h}^{r}\left(\xi ; U_{\mathrm{hom}}\right), \dot{v}_{h}^{r}\left(\xi ; U_{\mathrm{hom}}\right)\right)(1.20)$, 'touches down' on $\mathcal{M}$ near $\ell^{s} \cap T_{\text {down }}$ and remains exponentially close to $\ell^{s}$ (Theorem 2.1 in [3] with $N=1$ ). For $0<\nu<\nu_{\mathrm{SN}}$, where

$$
\nu=\nu_{\mathrm{SN}}(\varepsilon)=\frac{D}{\beta_{2}-1+D} \mu U_{\mathrm{SN}}^{2}+\mathcal{O}(\varepsilon) \text { with } U_{\mathrm{SN}}=\left(\frac{\beta_{2}-1}{\beta_{2}-1+D}\right)^{\frac{\beta_{2}-1}{2 D}} U_{\mathrm{hom}}+\mathcal{O}(\varepsilon)
$$

((1.21), (1.15), [6]), the intersections $T_{\text {off }} \cap \Gamma_{\nu}$ and $T_{\text {down }} \cap \Gamma_{\nu}(1.15)$ consist of two points (see Figure 2. Each symmetric pair of points in $T_{\text {off,down }} \cap \Gamma_{\nu}$ corresponds - again by construction $[3,6]$ - to a solution of (1.18) that is homoclinic to $\mathcal{M}$, i.e. $\nu \in\left(0, \nu_{\mathrm{SN}}\right)$ labels two families of homoclinic solutions, $\gamma_{\mathcal{M}, A}(\xi ; \nu)=\left(u_{\mathcal{M}, A}(\xi ; \nu), p_{\mathcal{M}, A}(\xi ; \nu), v_{\mathcal{M}, A}(\xi ; \nu), q_{\mathcal{M}, A}(\xi ; \nu)\right)$ and $\gamma_{\mathcal{M}, B}(\xi ; \nu)=$ $\left(u_{\mathcal{M}, B}(\xi ; \nu), p_{\mathcal{M}, B}(\xi ; \nu), v_{\mathcal{M}, B}(\xi ; \nu), q_{\mathcal{M}, B}(\xi ; \nu)\right)$. If $\left(U_{0, A}, \pm P_{0, A}\right)$ and $\left(U_{0, B}, \pm P_{0, B}\right)$ are the $(u, p)$-coordinates of $T_{\text {off,down }} \cap \Gamma_{\nu} \subset \mathcal{M}$, then the distinction between the orbits of A- or B-type is made by

$$
0<U_{0, B}(\nu)<U_{0, B}\left(\nu_{\mathrm{SN}}\right)=U_{\mathrm{SN}}=U_{0, A}\left(\nu_{\mathrm{SN}}\right)<U_{0, A}(\nu)<U_{0, A}(0)=U_{\mathrm{hom}}
$$

for $\nu \in\left(0, \nu_{\mathrm{SN}}\right)$. The orbits $\gamma_{\mathcal{M}, A, B}(\xi ; \nu)$ travel twice along the parts of $\Gamma_{\nu}$ in between $\left(U_{0, A, B},-P_{0, A, B}\right)$ and $\left(U_{0, A, B},+P_{0, A, B}\right)$. The $2 L$-periodic patterns $\left(U_{p, A, B}(\xi ; L), V_{p, A, B}(\xi ; L)\right)$ are - by construction - exponentially close to $\left(U_{\mathcal{M}, A, B}(\xi ; \nu), V_{\mathcal{M}, A, B}(\xi ; \nu)\right)$, i.e. the $u, v$-components of $\gamma_{\mathcal{M}, A, B}(\xi ; \nu)$, for all $\xi$ such that $u_{\mathcal{M}, A, B}(\xi ; \nu) \leq U_{0, A, B}$.

Theorem 1.7 Let $\varepsilon \ll 1$ and assume that (1.3) holds. Equation (1.18) possesses two oneparameter families of $2 L$-periodic orbits, $\gamma_{p, A}(\xi ; \nu)=\left(u_{p, A}(\xi ; \nu), p_{p, A}(\xi ; \nu), v_{p, A}(\xi ; \nu), q_{p, A}(\xi ; \nu)\right)$ and $\gamma_{p, B}(\xi ; \nu)=\left(u_{p, B}(\xi ; \nu), p_{p, B}(\xi ; \nu), v_{p, B}(\xi ; \nu), q_{p, B}(\xi ; \nu)\right)$ with positive $u, v$-coordinates, parameterized by $\nu \in\left(0, \nu_{\mathrm{SN}}\right)$ (1.24). For each $\nu, \gamma_{p, A, B}(\xi ; \nu)$ consist of a slow piece exponentially close to the part of $\Gamma_{\nu} \subset \mathcal{M}$ with $u<U_{0, A, B}$, where

$$
U_{0, A, B}(\nu)=\left(\tanh \left(\varepsilon^{2} \sqrt{\mu} L_{A, B}(\nu)\right)\right)^{\frac{\beta_{2}-1}{D}} U_{\text {hom }}
$$

(1.23), or equivalently,

$$
L_{A, B}(\nu)=\frac{1}{\varepsilon^{2} \sqrt{\mu}} \operatorname{arccosh}\left(\frac{U_{0, A, B}(\nu)}{U_{\min }(\nu)}\right)
$$

(1.17), and a fast jump away from $\mathcal{M} \mathcal{O}(\varepsilon)$ close to $\left(U_{0, A, B}, 0, v_{h}^{r}\left(\xi ; U_{0, A, B}\right), \dot{v}_{h}^{r}\left(\xi ; U_{0, A, B}\right)\right)$ (1.20). The orbits $\gamma_{p, A, B}(\xi ; \nu)$ intersect the $\{p=q=0\}$-plane twice, so that they have two internal reflection points at $\xi=\xi_{0}$ and at $\xi=\xi_{0}+L_{A, B}$.

If $\nu \downarrow 0,\left\|\gamma_{p, B}(\xi ; \nu)\right\| \rightarrow 0$ uniformly in $\xi$, while $\gamma_{p, A}(\xi ; \nu) \rightarrow \gamma_{h}(\xi)$, the homoclinic solution to $(0,0,0,0)$. If $\nu \uparrow \nu_{\mathrm{SN}}(1.24), \gamma_{p, A}(\xi ; \nu)$ and $\gamma_{p, B}(\xi ; \nu)$ merge in a saddle-node bifurcation of periodic orbits. For $0<\nu<\nu_{\mathrm{SN}}, U_{0, B}(\nu)<U_{\mathrm{SN}}<U_{0, A}(\nu)$ and $L_{0, B}(\nu)<L_{\mathrm{SN}}<L_{0, A}(\nu)$, where

$$
\begin{aligned}
U_{\mathrm{SN}} & =U_{\min }\left(\nu_{\mathrm{SN}}\right) \sqrt{\frac{\beta_{2}-1+D}{D}}+\mathcal{O}(\varepsilon), \quad \text { and } \\
\varepsilon^{2} L_{\mathrm{SN}} & =\frac{1}{\sqrt{\mu}} \operatorname{arccosh} \sqrt{\frac{\beta_{2}-1+D}{D}}+\mathcal{O}(\varepsilon)
\end{aligned}
$$

(1.24), (1.16).

The orbits $\gamma_{p, A}(\xi ; \nu), \gamma_{p, B}(\xi ; \nu)$ correspond to the $2 L$-periodic patterns $\left(U_{p, A}(\xi ; L), V_{p, A}(\xi ; L)\right)$, $\left(U_{p, B}(\xi ; L), V_{p, B}(\xi ; L)\right)$ of (1.2) with $L=L_{A, B}(\nu)$. The internal reflection points $\xi_{n}=\xi_{0}+$ $n L_{A, B}, n \in \mathbb{Z}$, are at the minima/maxima of $U_{p, A, B}(\xi ; L(\nu)), V_{p, A, B}(\xi ; L(\nu)) . A s L \rightarrow \infty$, the fundamental periodic pattern of type $A$ approaches the homoclinic pattern $\left(U_{h}(\xi), V_{h}(\xi)\right)$ uniformly in $\xi$, i.e.

$$
\sup _{\xi \in\left[\xi_{0}-L, \xi_{0}+L\right]}\left\|\left(U_{h}(\xi), V_{h}(\xi)\right)-\left(U_{p, A}(\xi ; L), V_{p, A}(\xi ; L)\right)\right\| \rightarrow 0 \text { as } L \rightarrow \infty
$$

$\left(U_{h}(\xi), V_{h}(\xi)\right)$ can also be denoted by $\left(U_{p, A}(\xi ; \infty), V_{p, A}(\xi ; \infty)\right)$. 
This Theorem is a combination of Theorem 3.1 and Corollary 3.2 (with $N=1$ ) in [6]. The relations $(1.25),(1.26)$ and (1.27) cannot be found explicitly in [6], but follow from a straightforward analysis using (1.15) and (1.16).

If we set $\xi_{0}=0$ at the maximum of the fast pulse, then the orbits $\gamma_{\mathcal{M}, B}(\xi)$ and $\gamma_{p, B}(\xi ; \nu)$ are exponentially close in $\varepsilon$ for $\xi \in\left[-2 L_{B}(\nu)+\sqrt{\varepsilon}, 2 L_{B}(\nu)-\sqrt{\varepsilon}\right.$ [6], i.e. almost for two full periods of $\gamma_{p, B}\left(\xi ; L_{B}\right)$. Note that the $\pm \sqrt{\varepsilon}$ are not optimal and could be improved to $\mathcal{O}(\varepsilon)$ quantities. However, as $\xi$ approaches $\pm 2 L_{B}$, i.e. as $\gamma_{\mathcal{M}, B}(\xi)$ and $\gamma_{p, B}(\xi ; \nu)$ approach $T_{\text {off,down }} \cap \Gamma_{\nu}$, then $\gamma_{p, B}(\xi ; \nu)$ jumps (again) away from $\mathcal{M}$, while $\gamma_{\mathcal{M}, B}(\xi)$ remains exponentially close to $\mathcal{M}$ for all $|\xi| \geq 2 L_{B}-\sqrt{\varepsilon}$. One can construct a solution $\gamma_{p, A B}(\xi ; \nu)$ of $(1.18)$ that is exponentially close to both $\gamma_{\mathcal{M}, B}(\xi)$ and $\gamma_{p, B}(\xi ; \nu)$ on this same $\xi$-interval, and that remains exponentially close to $\gamma_{\mathcal{M}, B}(\xi)$ for $\xi \in\left[-L_{B}(\nu)-L_{A}(\nu)+\sqrt{\varepsilon}, L_{B}(\nu)+L_{A}(\nu)-\sqrt{\varepsilon}\right]$, i.e. beyond the take off/touch down points of $\gamma_{p, B}(\xi ; \nu)$. This new solution $\gamma_{p, A B}(\xi ; \nu)$ takes off/touches down at the A-intersections of $T_{\text {off,down }} \cap \Gamma_{\nu}$, i.e. exponentially close to a $\gamma_{p, A}(\xi ; \nu)$. The $\gamma_{p, A B}(\xi ; \nu)$ is periodic with period $2 L_{A B}=2 L_{A}+2 L_{B}$.

Theorem 1.8 Let $\varepsilon \ll 1$ and assume that (1.3) holds. Equation (1.18) possesses a family of $2 L$-periodic orbits, $\gamma_{p, A B}(\xi ; \nu)=\left(u_{p, A B}(\xi ; \nu), p_{p, A B}(\xi ; \nu), v_{p, A B}(\xi ; \nu), q_{p, A B}(\xi ; \nu)\right)$ with positive $u, v$-coordinates, parameterized by $\nu \in\left(0, \nu_{\mathrm{SN}}\right) ; L=L_{A B}(\nu)=L_{A}+L_{B}$ with $L_{A, B}(\nu)$ as in Theorem 1.7. The orbits $\gamma_{p, A B}(\xi ; \nu)$ intersect the $\{p=q=0\}$-plane twice and have two internal reflection points at $\xi=\xi_{A}$ and at $\xi=\xi_{B}=\xi_{A}+L_{A}+L_{B}$. The orbit $\gamma_{p, A B}(\xi ; \nu)$ is exponentially close to an orbit $\gamma_{p, A}(\xi ; \nu)$ on the intervals $\left[\xi_{A}-L+n L+\sqrt{\varepsilon}, \xi_{A}+L+n L-\sqrt{\varepsilon}\right.$, $n \in \mathbb{Z}$, where $\gamma_{p, A}(\xi ; \nu)$ is translated such that $\xi=\xi_{A}$ is a reflection point of $\gamma_{p, A}(\xi ; \nu)$ at the center of an A-pulse, and exponentially close to an orbit $\gamma_{p, B}(\xi ; \nu)$ on intervals $\left[\xi_{B}-2 L_{B}+\right.$ $\left.n L+\sqrt{\varepsilon}, \xi_{B}+2 L_{B}+n L-\sqrt{\varepsilon}\right], n \in \mathbb{Z}$, where $\xi=\xi_{B}$ defines the center of the B-pulse of $\gamma_{p, B}(\xi ; \nu)$.

As $\nu \uparrow \nu_{\mathrm{SN}}, \gamma_{p, A B}(\xi ; \nu)$ merges with $\gamma_{p, A}(\xi ; \nu)$ (or $\left.\gamma_{p, B}(\xi ; \nu)\right)$, but with twice its period, since

$$
L_{A B}(\nu)=L_{A}(\nu)+L_{B}(\nu)>2 L_{\mathrm{SN}}
$$

for all $\nu \in\left(0, \nu_{\mathrm{SN}}\right)$, i.e. the saddle-node bifurcation of Theorem 1.7 can also be seen as a perioddoubling bifurcation.

The orbits $\gamma_{p, A B}(\xi ; \nu)$ correspond to the $2 L$-periodic patterns $\left(U_{p, A B}(\xi ; L), V_{p, A B}(\xi ; L)\right)$ in (1.2) with $L=L_{A B}(\nu)$. The internal reflection points $\xi_{n}=\xi_{A}+n L, n \in \mathbb{Z}$, are at the centers (maxima) of the alternating pulses of A-type ( $n$ even), or of B-type ( $n$ odd).

Again, a more general result has already been proven in [6] (Theorem 3.3). The fact that $L_{A B}(\nu)>2 L_{\mathrm{SN}}$ has not been explicitly noticed in [6], but follows from straightforward calculations.

\section{Stability of periodic patterns}

Let $\left(U_{p}(\xi), V_{p}(\xi)\right)$ be a periodic pulse solution to (1.2) as described in the previous section with minimal spatial period $2 L$. The ideas we will develop in this section are valid for arbitrary periodic solutions, both for the fundamental periodic solutions and for the periodic solutions of AB-type. After developing the general theory, we first study the stability of the fundamental solutions (Theorem 1.7) in the Sections 3-6 and then the stability of the solutions of AB-type in Section 7.

We investigate the linear stability of the periodic solution against perturbations in $B C\left(\mathbb{R}, \mathbb{R}^{2}\right)$, i.e. the space of bounded and uniformly continuous functions, and set

$$
U(\xi, t)=U_{p}(\xi)+u(\xi) e^{\lambda t} V(\xi, t)=V_{p}(\xi)+v(\xi) e^{\lambda t}
$$


After substitution into (1.2) and linearization we obtain

$$
\left\{\begin{array}{l}
u_{\xi \xi}=-\varepsilon^{2}\left[\alpha_{1} U_{p}^{\alpha_{1}-1} V_{p}^{\beta_{1}} u+\beta_{1} U_{p}^{\alpha_{1}} V_{p}^{\beta_{1}-1} v\right]+\varepsilon^{4}(\mu+\lambda) u \\
v_{\xi \xi}+\left[\beta_{2} U_{p}^{\alpha_{2}} V_{p}^{\beta_{2}-1}-(1+\lambda)\right] v=-\alpha_{2} U_{p}^{\alpha_{2}-1} V_{p}^{\beta_{2}} u
\end{array}\right.
$$

Note that we switched to the fast scale $\xi=x / \varepsilon$. Equivalently, we can write the eigenvalue equation as a 4-dimensional system,

$$
\dot{\phi}(\xi)=A_{p}(\xi ; \lambda, \varepsilon) \phi(\xi),
$$

where $\phi=\left(u, p=u_{\xi}, v, q=v_{\xi}\right)^{t}$. The matrix $A_{p}$ is given by

$$
A_{p}(\xi ; \lambda, \varepsilon)=\left(\begin{array}{cccc}
0 & 1 & 0 & 0 \\
-\varepsilon^{2} \alpha_{1} U_{p}^{\alpha_{1}-1} V_{p}^{\beta_{1}}+\varepsilon^{4}(\lambda+\mu) & 0 & -\varepsilon^{2} \beta_{1} U_{p}^{\alpha_{1}} V_{p}^{\beta_{1}-1} & 0 \\
0 & 0 & 0 & 1 \\
-\alpha_{2} U_{p}^{\alpha_{2}-1} V_{p}^{\beta_{2}} & 0 & -\beta_{2} U_{p}^{\alpha_{2}} V_{p}^{\beta_{2}-1}+(1+\lambda) & 0
\end{array}\right) .
$$

The theory for linear ordinary differential equations with periodic coefficients like (2.1) is well developed. This Floquet Theory leads to the concept of $\gamma$-eigenvalues [10].

Definition $2.1 \lambda \in \mathbb{C}$ is a $\gamma$-eigenvalue of eigenvalue problem (2.2) if (2.2) has a solution $\phi(\xi ; \lambda)$ that satisfies

$$
\phi(\xi+2 L ; \lambda)=\gamma \phi(\xi ; \lambda)
$$

for some $\gamma \in \mathbf{S}^{1}$. The corresponding eigenfunction $\phi(\xi ; \lambda)$ is called a $\gamma$-eigenfunction; $\gamma \in \mathbf{S}^{1}$ for which (2.4) is satisfied is called the Floquet multiplier of $\phi(\xi ; \lambda)$.

Note that if $\phi(\xi)$ satisfies condition (2.4) in one point, it automatically satisfies it in every point.

It was shown in [10] that any bounded solution to (2.2) can be written as a combination of $\gamma$-eigenfunctions and hence that the spectrum of the linearization around a period pattern consists entirely of $\gamma$-eigenvalues. In general, the spectrum of a linearized problem with periodic coefficients like (2.2) consists of a number of 'loops', where each loop is an image of $\mathbf{S}^{1}$. However, because of the reflection symmetry present in the particular system (2.2), this image will have a special structure.

The matrix $A_{p}(\xi ; \lambda)(2.3)$ inherits the reflection symmetry of the periodic solution $\left(U_{p}(\xi), V_{p}(\xi)\right)$, i.e. for every reflection point $\xi_{0}$ of the pulse solution we have $A_{p}\left(\xi_{0}+\eta ; \lambda\right)=A_{p}\left(\xi_{0}-\eta ; \lambda\right)$ for all $\lambda$ and all $\eta=\xi-\xi_{0}$. This immediately implies that if $\phi(\xi)$ is a solution to (2.2), the reflection of $\phi(\xi)$ in any reflection point is also a solution. We denote the reflection of $\phi(\xi)$ in the reflection point $\xi_{0}$ by $R \phi\left(\xi_{0}-\eta\right)$, where $\eta=\xi-\xi_{0}$ and $R$ is defined by

$$
R\left(\begin{array}{c}
u(\xi) \\
p(\xi) \\
v(\xi) \\
q(\xi)
\end{array}\right)=\left(\begin{array}{r}
u(\xi) \\
-p(\xi) \\
v(\xi) \\
-q(\xi)
\end{array}\right)
$$

By applying this reflection symmetry to a $\gamma$-eigenfunction, it follows that if $\lambda$ is a $\gamma$-eigenvalue then $\lambda$ is also a $\bar{\gamma}$-eigenvalue. Let $\phi(\xi)$ be a $\gamma$-eigenfunction associated to the $\gamma$-eigenvalue $\lambda$, i.e. $\phi(\xi)$ is a solution to $(2.2)$ that satisfies $\phi(\xi+2 L)=\gamma \phi(\xi)$. Then, setting $\xi_{0}=0$, the reflected solution $\hat{\phi}(\xi)=R \phi(-\xi)$ is also a solution of (2.2). Clearly this solution satisfies $\hat{\phi}(\xi+2 L)=R \phi(-\xi-2 L)=\frac{1}{\gamma} R \phi(-\xi)=\bar{\gamma} \hat{\phi}(\xi)$. Hence, $\hat{\phi}(\xi)$ is a $\bar{\gamma}$-eigenfunction and $\lambda$ is a $\bar{\gamma}$-eigenvalue.

The general results in [10], combined with the reflection symmetry in (2.2), lead to the following characterization of the spectrum of the linearization around periodic pulse solutions of the type analyzed in this paper. 
Proposition 2.2 The spectrum of the linearization around the periodic pulse solution $\left(U_{p}(\xi), V_{p}(\xi)\right)$ consists of a number of (degenerate) curves of $\gamma$-eigenvalues. Each curve can be parameterized by $\lambda=\lambda(\gamma)=\lambda(\bar{\gamma})$, where $\gamma \in \mathbf{S}^{1}$ is the Floquet multiplier of the associated $\gamma$-eigenfunction, i.e. the image of $\mathbf{S}^{1}$ covers each curve of $\gamma$-eigenvalues twice. The periodic pulse pattern is linearly stable, if apart from a translational 1-eigenvalue at the origin, every spectral curve is completely in the stable half plane $\{\operatorname{Re}[\lambda]<0\}$.

Remark 2.3 It is important to distinguish between two notions of (geometric) multiplicity of an eigenvalue $\lambda$. First, the $\gamma$-multiplicity of an eigenvalue $\lambda$ is defined as the number of independent (generalized) $\gamma$-eigenfunctions to (2.2) for one particular value of $\gamma$. On the other hand the (total) multiplicity of $\lambda$ as an element of the spectrum is given by the number of independent (generalized) eigenfunctions of (2.1), i.e. the number of independent bounded solutions to (2.1). It follows from the fact that any bounded solution to (2.1) can be written as a combination of $\gamma$-eigenfunctions, that the total multiplicity of $\lambda$ is given by the sum of $\gamma$-multiplicities over all $\gamma$ for which $\lambda$ is a $\gamma$-eigenvalue.

Remark 2.4 The spectral curves indicated in Proposition 2.2 are not necessary disjoint; two or more curves may intersect, may be connected at the endpoints of the curves, or may coincide partially or completely. However, in all cases it is still possible to parameterize the curves by the Floquet multiplier of the associated $\gamma$-eigenfunction.

Remark 2.5 In general, the reflection $R \phi\left(\xi_{0}-\eta\right)$ of a solution in a reflection point $\xi_{0}$ is different from the solution $\phi\left(\xi_{0}+\eta\right)$. However, in two special cases $R \phi\left(\xi_{0}-\eta\right)$ is not independent of $\phi\left(\xi_{0}+\eta\right)$, i.e. $R \phi\left(\xi_{0}-\eta\right)=\theta \phi_{0}(\xi+\eta)$ for some number $\theta$. First, a solution such that $R \phi\left(\xi_{0}-\eta\right)=\phi\left(\xi_{0}+\eta\right)(\theta=1)$ is called symmetric in the reflection point $\xi_{0}$. The evaluation of the symmetry condition for $\eta=0$ yields that $\phi(\xi)$ is symmetric in $\xi_{0}$ if and only if its $p$ and $q$-components vanish in $\xi_{0}$, i.e. if and only if $u(\xi)$ and $v(\xi)$ are even with respect to $\xi_{0}$. Second, a solution such that $R \phi\left(\xi_{0}-\eta\right)=-\phi\left(\xi_{0}+\eta\right)(\theta=-1)$ is called anti-symmetric in the reflection point $\xi_{0} ; \phi(\xi)$ is anti-symmetric in $\xi_{0}$ if and only if its $u$ - and $v$-components vanish in $\xi_{0}$, i.e. if and only if $u(\xi)$ and $v(\xi)$ are odd with respect to $\xi_{0}$. Note that it is not possible to have $\theta \neq \pm 1$. Symmetrical and anti-symmetrical solutions to (2.2) play an important role in the stability analysis of pulse patterns on a finite interval with homogeneous Neumann boundary conditions, see Section 8.

\subsection{The monodromy matrix and the Evans function}

Using the concept of $\gamma$-eigenvalues and $\gamma$-eigenfunctions, the stability problem of the periodic pattern $\left(U_{p}(\xi), V_{p}(\xi)\right)$ can be reduced to the problem of solving the linearized equations (2.2) over just one spatial period of the pattern, chosen to be the interval $[-L, L]$, with the boundary condition $\phi(L)=\gamma \phi(-L)$. This problem is studied using the monodromy matrix $M_{L}(\lambda, \varepsilon)$ associated to $(2.2)$, that is defined by

$$
\Psi(L ; \lambda, \varepsilon)=\Psi(-L ; \lambda, \varepsilon) M_{L}(\lambda, \varepsilon),
$$

where $\Psi(\xi ; \lambda, \varepsilon)$ is a fundamental matrix solution to $(2.2)$. The eigenvectors of $M_{L}(\lambda, \varepsilon)$ are related to the $\gamma$-eigenfunctions of (2.2). If $\mathbf{v}$ is an eigenvector of $M_{L}(\lambda, \varepsilon)$ with corresponding eigenvalue $\rho$, then $\phi(\xi)=\Psi(\xi) \mathbf{v}$ is a solution to $(2.2)$ which satisfies $\phi(L)=\rho \phi(-L)$. This argument can be reversed, if there exists a solution $\phi(\xi)$ to $(2.2)$, such that $\phi(L)=\rho \phi(-L)$, then $\rho$ is an eigenvalue of $M_{L}(\lambda)$. Therefore, $\lambda$ is a $\gamma$-eigenvalue if and only if $M_{L}(\lambda, \varepsilon)$ has an eigenvalue $\gamma \in \mathbf{S}^{1}$.

The eigenvalues $\rho$ of the monodromy matrix depend on $\lambda$. Since the elements of $M_{L}(\lambda)$ are analytic functions of $\lambda$, the $\rho(\lambda)$ are at least continuous in $\lambda$. The eigenvalues of $M_{L}(\lambda)$ are 
implicitly defined as the roots of the characteristic polynomial

$$
P(\lambda, \rho ; L, \varepsilon)=\operatorname{det}\left[M_{L}(\lambda ; \varepsilon)-\rho \mathrm{I}\right] .
$$

Following $[10,11]$, we define the Evans function $\mathcal{D}(\lambda, \gamma ; L, \varepsilon)$ associated to the linear eigenvalue problem (2.2) as the restriction of $P(\lambda, \rho)$ to $\rho=\gamma \in \mathbf{S}^{1}$,

$$
\mathcal{D}(\lambda, \gamma ; L, \varepsilon)=\operatorname{det}\left[M_{L}(\lambda ; \varepsilon)-\gamma \mathrm{I}\right] .
$$

Since $\mathcal{D}(\lambda, \gamma ; L)=0$ if and only if $\gamma$ is an eigenvalue of $M_{L}(\lambda)$, the zeroes of the Evans function correspond to $\gamma$-eigenvalues of (2.2). The elements of $M_{L}(\lambda)$ are analytic functions of $\lambda$, hence $\mathcal{D}(\lambda, \gamma ; L)$ is an analytic function of both $\lambda$ and $\gamma$. The order of a zero $\lambda_{0}$ of $\mathcal{D}(\lambda, \gamma ; L)$ with $\gamma$ fixed gives the algebraic $\gamma$-multiplicity of the $\gamma$-eigenvalue $\lambda_{0}$. Note that this is not necessarily the same as the algebraic multiplicity of $\gamma$ as an eigenvalue of $M_{L}\left(\lambda_{0}\right)$, since the latter is defined as the order of the zero for fixed $\lambda$. See [10] for an example where the two multiplicities are different. On the other hand, the geometric multiplicity of $\gamma$ as an eigenvalue of $M_{L}\left(\lambda_{0}\right)$ is always equal to the geometric $\gamma$-multiplicity of $\lambda_{0}$.

Although the matrix $M_{L}(\lambda, \varepsilon)$ depends on the choice of the fundamental matrix solution in (2.6), the eigenvalues of $M_{L}(\lambda, \varepsilon)$ are independent of that choice. In fact, if $\tilde{\Psi}(\xi)$ and $\Psi(\xi)$ denote two fundamental matrix solutions, there exists an invertible constant matrix $C$ such that $\tilde{\Psi}(\xi)=\Psi(\xi) C$ and we can thus write $\tilde{M}_{L}=\tilde{\Psi}(-L)^{-1} \tilde{\Psi}(L)=C^{-1} M_{L} C$. Hence, the eigenvalues of $M_{L}$ are independent of the choice of the fundamental matrix solution in (2.6), so that $\mathcal{D}(\lambda, \gamma ; L)$ does not depend on this choice. Similarly, the monodromy matrix can be expressed on a different basis, without changing its eigenvalues. In particular, we will express $M_{L}$ with respect to the basis $V=V(\lambda)$ spanned by the 4 columns of $\Psi(-L), \Psi(-L)=I$ with respect to $V$, so that $M_{L, v}=\Psi(L)$ (with respect to $V$ ). With a suitable choice of the fundamental matrix solution in (2.6), the monodromy matrix takes a form that simplifies the evaluation of the Evans function considerably.

The monodromy matrix $M_{L}$ has 4 eigenvalues $\rho_{i}$, counting their algebraic multiplicity. Therefore, the algebraic multiplicity of any $\lambda \in \mathbb{C}$ is at most 4 . Alternatively, the Evans function can be expressed as

$$
\mathcal{D}(\lambda, \gamma ; L, \varepsilon)=\prod_{i=1}^{4}\left(\rho_{i}(\lambda, \varepsilon)-\gamma\right),
$$

where $\rho_{i}(\lambda ; \varepsilon)$ denote the eigenvalues of $M_{L}$. Though this form stresses the relation between $\gamma$-eigenvalues and the eigenvalues of $M_{L}(\lambda)$, its use is restricted to situations where one has expressions for the eigenvalues of $M_{L}(\lambda)$. It follows from Abels theorem and the fact that $\operatorname{Tr}\left[A_{p}(\xi)\right]=0(2.3)$ that the 4 eigenvalues $\rho_{i}$ of $M_{L}(\lambda, \varepsilon)$ satisfy $\rho_{1} \rho_{2} \rho_{3} \rho_{4}=1$. We can deduce a stronger result using the reversibility symmetry in (2.2). If (2.2) has a solution with $\phi(L)=\rho \phi(-L)$, then there also exists a solution satisfying $\phi(L)=\frac{1}{\rho} \phi(-L)$. These two solutions correspond to the eigenvalues $\rho$ and $1 / \rho$ of $M_{L}(\lambda)$. Hence, the eigenvalues of $M_{L}(\lambda)$ come in pairs $\rho$ and $1 / \rho$. This implies that the algebraic multiplicity of \pm 1 is always 2 or 4. The geometric multiplicity of an eigenvalue \pm 1 can be odd. Also note, that if $\lambda$ is not a $\gamma$-eigenvalue, $M_{L}(\lambda)$ has two eigenvalues inside $\mathbf{S}^{1}$ and two eigenvalues outside $\mathbf{S}^{1}$. For real $\lambda$, all elements of $M_{L}(\lambda)$ are real. Therefore, the eigenvalues of $M_{L}(\lambda)$ are real or come as a pair of complex conjugated eigenvalues $\rho$ and $\bar{\rho}$, and thus, if $\rho$ is an eigenvalue of $M_{L}(\lambda)$ with $\lambda \in \mathbb{R}$, so are $1 / \rho, \bar{\rho}$ and $1 / \bar{\rho}$. Therefore, if $M_{L}(\lambda)$ has one non-real eigenvalue $\rho$ with $|\rho| \neq 1$, the other 3 eigenvalues are automatically given.

Remark 2.6 The $\gamma$-eigenfunctions, monodromy matrix and the Evans function are defined with respect to the minimal period $2 L$. However, in some cases, like the analysis of an ABpattern close to the saddle-node bifurcation (Section 7), or in the restriction to bounded domains (Section 8) it is useful to consider the periodic solution and the associated linear eigenvalue problem as $2 m L$-periodic, for some integer $m \geq 2$. Since the $2 m L$-periodic eigenvalue problem describes the same stability problem as the $2 L$-periodic eigenvalue problem, they share the 
same spectrum. On the other hand, the description of the spectrum in terms of $\gamma$-eigenvalues is different for the $2 m L$-periodic eigenvalue problem. A $\gamma$-eigenfunction $\phi(\xi)$ for the $2 L$-periodic eigenvalue problem, satisfies $\phi(\xi+2 m L)=\gamma^{m} \phi(\xi)$, hence it is a $\gamma^{m}$-eigenfunction of the $2 m L$ periodic eigenvalue problem. For each curve $\lambda=\lambda(\gamma)$ of $\gamma$-eigenvalues in the spectrum of the $2 L$-periodic eigenvalue problem, the spectrum of the $2 m L$-periodic eigenvalue problem contains $m$ connected curves of $\gamma$-eigenvalues. Also, the monodromy matrices and the Evans functions are different. Since $M_{m L}(\lambda)=\left(M_{L}(\lambda)\right)^{m}$, the monodromy matrices $M_{m L}(\lambda)$ and $M_{L}(\lambda)$ have the same eigenvectors and their eigenvalues are related by $\rho_{m L}=\rho_{L}^{m}$. Hence the zeroes of the Evans function $\mathcal{D}(\lambda, \gamma ; m L)$ occur for the same values of $\lambda$ as those of $\mathcal{D}(\lambda, \gamma ; m L)$, but for different values of $\gamma$.

\section{Stability analysis for fundamental patterns}

The ideas in the previous section can be applied to any of the periodic pulse solutions discussed in the Introduction. In this section, we study the linearized stability problem for fundamental periodic pulse solutions (Theorem 1.7), whereas the stability of the periodic solutions of ABtype will be treated in Section 7 .

Using the translational invariance, we shift the solution so that one of the pulses is at the origin. The stability problem is studied on the symmetric interval $[-L,+L]$ around the pulse, i.e. the two boundaries are halfway to the next pulse. Note that with this choice both the endpoints, $\pm L$, and the origin are points of symmetry of the periodic pulse solution. Around the pulse in the origin we define the pulse region $\mathcal{P}_{0}$, that divides the interval into three parts. The width of $\mathcal{P}_{0}$ is chosen such that $V_{p}(\xi ; L)$ is exponentially small everywhere outside $\mathcal{P}_{0}$ but $U_{p}(\xi ; L)$ is to leading order constant over the pulse interval. This (for instance) leads to the following choices

$$
\mathcal{I}_{-}=\left[-L,-\frac{1}{\sqrt{\varepsilon}}\right], \quad \mathcal{P}_{0}=\left(-\frac{1}{\sqrt{\varepsilon}}, \frac{1}{\sqrt{\varepsilon}}\right), \quad \mathcal{I}_{+}=\left[\frac{1}{\sqrt{\varepsilon}}, L\right],
$$

measured in the fast $(\xi-)$ scale. In the outer regions $\mathcal{I}_{+}$and $\mathcal{I}_{-}$, we can approximate the matrix $A_{p}(\xi ; \lambda, \varepsilon)(2.3)$ up to exponentially small error by the limit matrix

$$
A_{\infty}(\lambda, \varepsilon)=\left(\begin{array}{cccc}
0 & 1 & 0 & 0 \\
\varepsilon^{4}(\lambda+\mu) & 0 & 0 & 0 \\
0 & 0 & 0 & 1 \\
0 & 0 & 1+\lambda & 0
\end{array}\right)
$$

The eigenvalues and eigenvectors of this matrix are given by

$$
\begin{array}{ll}
\Lambda_{1,4}(\lambda)= \pm \Lambda^{f}(\lambda) \equiv \pm \sqrt{1+\lambda}, & \Lambda_{2,3}(\lambda, \varepsilon)= \pm \Lambda^{s}(\lambda, \varepsilon) \equiv \pm \varepsilon^{2} \sqrt{\mu+\lambda} \\
E_{1,4}(\lambda)=E_{ \pm}^{f}(\lambda) \equiv\left(0,0,1, \pm \Lambda^{f}\right)^{t}, & E_{2,3}(\lambda, \varepsilon)=E_{ \pm}^{s}(\lambda, \varepsilon) \equiv\left(1, \pm \varepsilon^{2} \sqrt{\mu+\lambda}, 0,0\right)^{t}
\end{array}
$$

Many of the results in this section require that $A_{\infty}$ is hyperbolic, i.e. that its eigenvalues have non-zero real part. This is, by definition, the case for $\lambda \in \mathbb{C}_{e}$ (1.7). Moreover, the eigenvalues of $A_{\infty}$ satisfy $\left|\Lambda^{s}\right| \ll\left|\Lambda^{f}\right|$ in $\mathbb{C}_{e}$, i.e. equation (2.1) exhibits a natural splitting in slow and fast solutions.

Remark 3.1 The fundamental periodic solution $\left(U_{p, A}(\xi ; L), V_{p, A}(\xi ; L)\right)$ restricted to $[-L, L]$ converges to the homoclinic pulse solution $\left(U_{h}(\xi), V_{h}(\xi)\right)$ on $\mathbb{R}$ as $L \rightarrow \infty$ (Theorem 1.7). The linearization around $\left(U_{h}(\xi), V_{h}(\xi)\right)$ is given by

$$
\dot{\phi}(\xi)=A_{h}(\xi ; \lambda, \varepsilon) \phi(\xi) .
$$

An eigenfunction of the linearization around $\left(U_{h}(\xi), V_{h}(\xi)\right)$ is a solution to $(3.3)$ that decays to zero as $|\xi| \rightarrow \infty$. The matrix $A_{h}(\xi ; \lambda)$ can be obtained by taking the limit $L \rightarrow \infty$ in $A_{p}(\xi ; \lambda)$. By Theorem 1.7, $\left\|A_{p}(\xi)-A_{h}(\xi)\right\| \rightarrow 0$ uniformly in $\xi \in[-L, L]$ for $L \rightarrow \infty ;\left\|A_{p}(\xi)-A_{h}(\xi)\right\|$ 
is exponentially small (in $L$ ) outside the pulse region. Thus, the results of [11, 27] on the stability of nearly homoclinic periodic waves can be applied to our problem (see Section 6.1). The analysis of the periodic eigenvalue problem (2.1) has many elements in common with the analysis of the homoclinic eigenvalue problem (3.3) in [3]. Therefore, we will refer to [3] and [4] at various places in the following analysis for further details.

\subsection{Fast solutions and the fast reduced limit problem}

Following Section 2.1, we choose a suitable fundamental matrix solution to express the monodromy matrix. Since $A_{p}(\xi)$ is exponentially close to the limit matrix $A_{\infty}$ on the outer regions, solutions to (2.2) behave like combinations of $E_{ \pm}^{f} e^{ \pm \Lambda^{f} \xi}$ and $E_{ \pm}^{s} e^{ \pm \Lambda^{s} \xi}$ on $\mathcal{I}_{-}$and $\mathcal{I}_{+}$. Therefore, it is natural to choose a fundamental matrix solution for the calculation of $M_{L}(\lambda)$ that consists (to leading order) of the 4 solutions, defined by the boundary conditions $\phi_{i}(-L)=\mathrm{E}_{i} e^{-\Lambda_{i} L}$, $i=1, \ldots, 4$.

Lemma 3.2 Let $\lambda \in \mathbb{C}_{e}$ (1.7) and let $\phi_{1}^{f}(\xi ; \lambda, \varepsilon)$ be the solution to (2.2) that satisfies $\phi_{1}^{f}(-L)=$ $e^{-\Lambda^{f} L} E_{+}^{f}$. Then, there exist an analytic (transmission) function $t^{f}(\lambda, \varepsilon)$ and a positive number $C$, such that

$$
e^{-\Lambda^{f} L} \phi_{1}^{f}(+L ; \lambda, \varepsilon)=t^{f}(\lambda, \varepsilon) E_{+}^{f}+\mathcal{O}\left(e^{-C L}\right),
$$

where the $\mathcal{O}\left(e^{-C L}\right)$ rest term is spanned by $\left\{E_{-}^{f}, E_{+}^{s}, E_{-}^{s}\right\}$.

Proof. This is a natural result since a solution to $(2.2)$ grows as $e^{\Lambda^{f} \xi}$ on $\mathcal{I}_{+}$in general. A full proof of this statement involves the Elephant Trunk procedure [12]. For this construction, there is no essential difference between the case $\xi \in \mathbb{R}$ and $\xi \in[-L, L]$; we refer to $[12,3,4]$ for the details.

We define $\phi_{2}^{f}(\xi ; \lambda, \varepsilon)=R \phi_{1}^{f}(-\xi ; \lambda, \varepsilon)(2.5)$ as the second independent solution of $\Psi(\xi ; \lambda, \varepsilon)$. Note that if $t^{f}(\lambda, \varepsilon) \neq 0, \phi_{2}^{f}(-L)=t^{f} e^{\Lambda^{f} L} E_{-}^{f}$ (to leading order), i.e. a multiple of $E_{-}^{f}$.

In the limit $L \rightarrow \infty$, the solution $\phi_{1}^{f}(\xi)$ corresponds to the solution $\phi_{1}(\xi)$ of the homoclinic linear stability problem (3.3) as defined in Lemma 3.5 of [3]. This solution $\phi_{1}(\xi)$ was defined as the unique solution of the linear eigenvalue problem, that decays to 0 as $e^{-\Lambda^{f}|\xi|}$ as $\xi \rightarrow-\infty$. In this limit, the transmission function $t^{f}(\lambda, \varepsilon)$ corresponds to the fast transmission function $t_{1}(\lambda, \varepsilon)$ as defined in Lemma 3.6 in [3].

Obviously, the solution $\phi_{1}^{f}$ is not a $\gamma$-eigenfunction. Instead, the fact that $\phi_{1}^{f}(L)$ is exponentially large and $\phi_{1}^{f}(-L)$ is exponentially small suggests that there exists a solution to (2.2) such that $\phi^{*}(L)=\rho \phi^{*}(-L)$, with $\rho=\mathcal{O}\left(e^{2 \Lambda L}\right)$. It is necessary for the forthcoming analysis to obtain a more detailed knowledge of the transmission function $t^{f}(\lambda)$, which can be obtained by studying the fast reduced limit problem (1.5).

We approach this problem in two ways. First, since taking the limit $\varepsilon \rightarrow 0$ means that $L=\mathcal{O}\left(1 / \varepsilon^{2}\right) \rightarrow \infty$, we consider this problem as that of a single homoclinic pulse on $\mathbb{R}$. This means that we determine the homoclinic pulse solution $\bar{V}_{h}(\xi)$ of $(1.5)$ and study its linear stability. This approach has the advantage that we can obtain explicit results on the location of the spectrum. Alternatively, we consider the wavelength $L$ as an independent large parameter and look for periodic pulse solutions to (1.5) of period $L$. The stability analysis of this reduced periodic solution gives insight in the stability issue of the periodic solutions of the full problem. As we will see below, the two ways of looking at the problem are closely connected.

The reduced equation is rescaled by setting $\bar{V}(\xi)=U_{0}^{-\alpha_{2} /\left(\beta_{2}-1\right)} W(\xi)$, so that the differential equation for $W$ reads

$$
W_{t}=W_{\xi \xi}-W+W^{\beta_{2}} .
$$


This equation has a stationary homoclinic pulse solution $w_{h}\left(\xi ; \beta_{2}\right)$, given by $(1.20)$. The linear stability of this pulse solution is determined by the linearized equation

$$
\left(\mathcal{L}^{r}-\lambda\right) w \equiv w_{\xi \xi}+\left[\beta_{2}\left(w_{h}(\xi)\right)^{\beta_{2}-1}-(1+\lambda)\right] w=0,
$$

which has the form of a Schrödinger equation with a sech-squared potential. The position of part of the spectrum can be predicted, because the derivative $\dot{w}_{h}(\xi)$ of the homoclinic pulse solution is an eigenfunction corresponding to $\lambda=0$. Since $\dot{w}_{h}(\xi)$ is an odd function and it has one zero, there must exist an eigenfunction that is even and without zeroes. This even eigenfunction corresponds to a positive eigenvalue.

Although the exact location of the eigenvalues of (3.4) can be obtained using methods from classical mathematical physics (see for example [22]), we follow a somewhat different approach here. Therefore, we write (3.4) as the system

$$
\dot{\psi}=A^{r}(\xi) \psi \text { with } \psi(\xi)=(w(\xi), \dot{w}(\xi))^{t},
$$

where the matrix $A^{r}(\xi)$ is given by

$$
A^{r}(\xi)=\left(\begin{array}{cc}
0 & 1 \\
-\beta_{2}\left(w_{h}(\xi)\right)^{\beta_{2}-1}+(1+\lambda) & 0
\end{array}\right) .
$$

Alternatively, the matrix $A^{r}$ can be obtained by taking the limit of the lower diagonal $2 \times 2$ block of $A_{p}(\xi)(2.3)$ and scaling out $U_{0}$. For $|\xi| \gg 1$, the matrix $A^{r}(\xi)$ is exponentially close to the limit matrix

$$
A_{\infty}^{r}=\left(\begin{array}{cc}
0 & 1 \\
1+\lambda & 0
\end{array}\right)
$$

that has eigenvalues $\Lambda_{ \pm}^{r}= \pm \Lambda^{f}$ and eigenvectors $\mathrm{E}_{ \pm}^{r}=\left(1, \pm \Lambda^{f}\right)^{t}$, with $\Lambda^{f}$ as in (3.2).

Consider the unique solution $\psi_{1}(\xi)$ to $(3.5)$ that satisfies $\lim _{\xi \rightarrow-\infty} \psi_{1}(\xi) e^{-\Lambda^{f} \xi}=\mathrm{E}_{+}^{r}$. In general, this solution grows as $e^{\Lambda^{f} \xi}$ for $\xi \gg 1$ and, similar to the behavior of $\phi_{1}^{f}$ for $\xi \gg 1$, defined in Lemma 3.2, there exists an analytic transmission function $t^{r}(\lambda)$ such that $\lim _{\xi \rightarrow \infty} \psi_{1}(\xi) e^{-\Lambda^{f} \xi}=t^{r}(\lambda) \mathrm{E}_{+}^{r}[3]$. Hence $\psi_{1}(\xi)$ is bounded as $\xi \rightarrow \infty$ if and only if $t^{r}(\lambda)=0$ and thus the position of the eigenvalues of the stability problem associated to the homoclinic pulses corresponds to the zeroes of $t^{r}(\lambda)$. The transmission function $t^{r}(\lambda)$ can be calculated explicitly using a transformation of (3.4) to a hypergeometric equation, see [3] and Section 4.

Lemma 3.3 All zeroes of the transmission function $t^{r}(\lambda)$ associated to the fast reduced limit problem (3.4) are given by $\lambda=\lambda_{j}^{r}$ with

$$
\lambda_{j}^{r}=\frac{1}{4}\left[\beta_{2}+1-j\left(\beta_{2}-1\right)\right]^{2}-1, \quad \text { for } j=0,1, \ldots, J-1,
$$

with $J$ such that

$$
J-1<\frac{\left(\beta_{2}+1\right)}{\left(\beta_{2}-1\right)} \leq J
$$

Note that $\lambda_{1}^{r}=0$ and that $\lambda_{0}^{r}>0$ as was predicted above.

Alternatively, we study the stability of spatially periodic solutions to (1.5) with period $L \gg 1$. Since the period of these pattern is long, the spatially periodic solutions $w_{p}(\xi)$ are close to the homoclinic pulse solution $w_{h}(\xi)$ on $[-L, L]$. The linearization around $w_{p}(\xi)$ is given by (3.4), or by the system $(3.5)$, with $w_{h}(\xi)$ replaced by $w_{p}(\xi)$. To this system we associate a $2 \times 2$ monodromy matrix $M_{L}^{r}(\lambda)$, defined by

$$
\Psi^{r}(L ; \lambda)=\Psi^{r}(-L ; \lambda) M_{L}^{r}(\lambda),
$$


where $\Psi^{r}(\xi ; \lambda)$ is a fundamental matrix solution to the reduced eigenvalue problem, and the Evans function

$$
\mathcal{D}^{r}(\lambda, \gamma ; L)=\operatorname{det}\left[M_{L}^{r}(\lambda)-\gamma I_{2}\right] .
$$

As the columns of the fundamental matrix solution $\Psi^{r}(\xi)$, we choose the two solutions $\psi_{1}^{p}(\xi)$ and $\psi_{2}^{p}(\xi)$, that satisfy

$$
\psi_{1}^{p}(-L)=e^{-\Lambda^{f} L} E_{+}^{r} \text { and } \psi_{2}^{p}(\xi)=R \psi_{1}^{p}(-\xi),
$$

where $R$ is the 2-dimensional equivalent of the reflection operator $R$ introduced in Section 2 . Note that since $\psi_{1}$ decays to 0 as $e^{\Lambda^{f} \xi}$ as $\xi \rightarrow-\infty$, it is exponentially close (in $L$ ) to $\psi_{1}^{p}$ if $\xi=-L$. For general $\lambda$, the solution $\psi_{1}^{p}$ grows as $e^{\Lambda^{f} \xi}$ for $\xi \gg 1$ and therefore, we can define an analytic transmission function $t_{p}^{r}(\lambda)$ and a positive number $C=C(\lambda)>0$ such that $\psi_{1}^{p}(L) e^{-\Lambda^{f} L}=t_{p}^{r}(\lambda) E_{+}^{r}+\mathcal{O}\left(e^{-C L}\right) E_{-}^{r}$, as in Lemma 3.2. It is clear that the two transmission functions $t_{p}^{r}(\lambda)$ and $t^{r}(\lambda)$ are closely related. Therefore, note that the periodic solution $V^{r}(\xi)$ is uniformly exponentially close to the homoclinic solution $V_{p}^{r}(\xi)$ on $[-L, L]$ and that $\psi_{1}(-L)$ is exponentially close to $\psi_{1}^{p}(-L)$. Hence, the two transmission functions are exponentially close to each other. The monodromy matrix can be expressed on the basis $V^{r}=\left\{\psi_{1}^{p}(-L), \psi_{2}^{p}(-L)\right\}$. Therefore, we write

$$
\begin{aligned}
& \psi_{1}^{p}(L)=m_{11}^{r} \psi_{1}^{p}(-L)+m_{21}^{r} \psi_{2}^{p}(-L) \\
& \psi_{2}^{p}(L)=m_{12}^{r} \psi_{1}^{p}(-L)+m_{22}^{r} \psi_{2}^{p}(-L)
\end{aligned}
$$

with $\psi_{1}^{p}(-L)=e^{-\Lambda^{f} L} E_{+}^{r}\left(\right.$ Lemma 3.2) and $\psi_{2}^{p}(-L)=R \psi_{1}^{p}(L)=t_{p}^{r} e^{\Lambda^{f} L} E_{-}^{r}+\mathcal{O}\left(e^{\left(\Lambda^{f}-C\right) L}\right) E_{+}^{r}$. Since $\psi_{1}^{p}(L)=t_{p}^{r} e^{\Lambda^{f} L} E_{+}^{r}+\mathcal{O}\left(e^{\left(\Lambda^{f}-C\right) L}\right) E_{-}^{r}$ and $\psi_{1}^{p}$ does not contain an $E_{-}^{r}$-term, we have $m_{21}^{r}=\mathcal{O}\left(e^{-C L}\right)$. Furthermore, by looking at the $E_{+}^{r}$ terms in the first line of (3.8), we find $m_{11}^{r}=t_{p}^{r} e^{2 \Lambda^{r} L}+$ h.o.t. Following a similar reasoning for $\phi_{2}^{p}(L)$, we find to leading order

$$
M_{L}^{r}(\lambda)=\left(\begin{array}{ll}
m_{11}^{r} & m_{12}^{r} \\
m_{21}^{r} & m_{22}^{r}
\end{array}\right)=\left(\begin{array}{cc}
t_{p}^{r}(\lambda) e^{2 \Lambda^{f} L} & \mathcal{O}\left(e^{-C L}\right) \\
\mathcal{O}\left(e^{-C L}\right) & \frac{1}{t_{p}^{r}(\lambda)} e^{-2 \Lambda^{f} L}
\end{array}\right)
$$

The exact expressions of the elements are $M_{L}^{r}(\lambda)$ are not needed to get leading order expressions of its eigenvalues. Due to the reversibility symmetry in the problem, we know that $\operatorname{det}\left[M_{L}^{r}\right]=1$. Since $\operatorname{Tr}\left[M_{L}^{r}\right]=\mathcal{O}\left(e^{2 \Lambda^{f} L}\right)$, the eigenvalues of the monodromy matrix are given by

$$
\rho_{1}^{r}(\lambda)=t_{p}^{r}(\lambda) e^{2 \Lambda^{f} L} \text { and } \rho_{2}^{r}(\lambda)=\frac{1}{\rho_{1}^{r}(\lambda)}
$$

(up to exponentially small errors in $L$ ). Hence, there are no $\gamma$-eigenvalues to the reduced eigenvalue problem if $t_{p}^{r}(\lambda)=\mathcal{O}(1)$. However, the above leading order analysis breaks down if $t_{p}^{r}(\lambda)$ becomes exponentially small, which implies that $\gamma$-eigenvalues of the reduced periodic eigenvalue problem may occur if $t_{p}^{r}(\lambda)$ is exponentially small.

Lemma 3.4 Let $\lambda_{j}^{r}$ be given by (3.6). For each $0 \leq j \leq J-1$ there exists a curve of $\gamma$ eigenvalues $\lambda_{j}^{r}(\gamma)$ with $\left|\lambda_{j}^{r}-\lambda_{j}^{r}(\gamma)\right|<e^{-C L}$ for some constant $C>0$. There are no other $\gamma$-eigenvalues in (3.4).

This Lemma can be proved with the methods developed here, however, this result also follows directly from the literature [11]. The Lemma implies that every periodic pulse solution to (1.5) with large enough spatial period is unstable (this is a special case of a well-known result, see $[23,11])$.

The two reduced transmission functions $t^{r}(\lambda)$ and $t_{p}^{r}(\lambda)$ are, by construction, leading order approximations of $t^{f}(\lambda)$ if $\lambda$ is not close to the reduced eigenvalues $\lambda_{j}^{r}, 0 \leq j \leq J-1(3.6)$, i.e. if $\lambda \in \mathbb{C}_{r}$ (1.8). 
Lemma 3.5 Let $\lambda \in \mathbb{C}_{r}$, then $t^{f}(\lambda)=t^{r}(\lambda)+\mathcal{O}(\varepsilon)$ uniformly in $\lambda$, so that $t^{f}(\lambda) \neq 0$ and $\mathcal{O}(1)$ with respect to $\varepsilon$ for $\lambda \in \mathbb{C}_{r}$.

Proof. As for Lemma 3.2, the proof of the statement $t^{f}(\lambda)=t_{p}^{r}(\lambda)+\mathcal{O}(\varepsilon)$ is completely analogous to the proof of the corresponding result for the stability of localized homoclinic pulses on $\mathbb{R}$, therefore we again refer to $[12,3,4]$ for the technical details. Since by construction $\left|t_{p}^{r}(\lambda)-t^{r}(\lambda)\right|<e^{-C L}$ for some $C>0$ and $L=\mathcal{O}\left(1 / \varepsilon^{2}\right)$, the Lemma follows from Lemma 3.3.

The relation between the fast ingredients of the Evans function $\mathcal{D}(\lambda, \gamma ; \varepsilon)$ and those of the fast reduced limit problem are much more subtle for $\lambda$ close to $\lambda_{j}^{r}$, and will be discussed in detail in Sections 4.3 and 5.

\subsection{The slow solutions}

In the previous section, we constructed two fast solutions $\phi_{1,2}^{f}$ to (2.2), which followed to leading order the behavior of the reduced equation. In this section, we determine a special set of two solutions $\phi_{1,2}^{s}$ that are independent of the two fast solutions. In general, a solution grows as the most unstable eigenmode $\left(e^{\Lambda^{f}|\xi|}\right)$ in the outer regions ( for $\lambda \in \mathbb{C}_{e}$ ). However, it was derived in [3] for the stability analysis of the homoclinic pulse that there exist solutions to the linearized homoclinic equations, that do not grow as $e^{\Lambda^{f}}|\xi|$ as $\xi \rightarrow \pm \infty$. Furthermore, it was shown in [3] that the behavior of these solutions determines the location of the eigenvalues associated to the stability of the pulse solutions. Therefore, we will also impose the condition on the slow solutions that they do not grow as the most unstable eigenmode.

Moreover, we impose a second condition on the slow solutions $\phi_{1,2}^{s}$; the subspace of solutions $\Phi^{s}(\xi ; \lambda)=\operatorname{span}\left\{\phi_{1}^{s}(\xi ; \lambda), \phi_{2}^{s}(\xi ; \lambda)\right\}$ must be invariant (as a subspace) under translation over one spatial period $2 L$, i.e. we require that $\phi_{1,2}^{s}$ satisfy

$$
\operatorname{span}\left\{\phi_{1}^{s}(\xi+2 L), \phi_{2}^{s}(\xi+2 L)\right\}=\operatorname{span}\left\{\phi_{1}^{s}(\xi), \phi_{2}^{s}(\xi)\right\}
$$

for all $\xi \in \mathbb{R}$. Since $A_{p}(\xi ; \lambda)(2.3)$ is $2 L$-periodic in $\xi, \phi(\xi+2 L)$ is a solution of $(2.2)$ if $\phi(\xi)$ is. It follows (by straightforward linear algebra) that it is always possible to choose two independent solutions of (2.2), such that they satisfy condition (3.10). However, together with the condition that $\phi_{1}^{s}$ and $\phi_{2}^{s}$ do not grow as $e^{\Lambda^{f}|\xi|}, \phi_{1}^{s}$ and $\phi_{2}^{s}$ are determined uniquely, as we will see in Lemma 3.6 below.

By $(3.10), \phi_{1,2}^{s}(L) \in \Phi^{s}(L ; \lambda)$ can be written as a linear combination of $\phi_{1,2}^{s}(-L)$, i.e. there exist coefficients $m_{i j}$ such that

$$
\begin{aligned}
& \phi_{1}^{s}(L ; \lambda, \varepsilon)=m_{11}(\lambda, \varepsilon) \phi_{1}^{s}(-L ; \lambda, \varepsilon)+m_{21}(\lambda, \varepsilon) \phi_{2}^{s}(-L ; \lambda, \varepsilon) \\
& \phi_{2}^{s}(L ; \lambda, \varepsilon)=m_{12}(\lambda, \varepsilon) \phi_{1}^{s}(-L ; \lambda, \varepsilon)+m_{22}(\lambda, \varepsilon) \phi_{2}^{s}(-L ; \lambda, \varepsilon)
\end{aligned} .
$$

Due to the $2 L$-periodicity and the linearity of $(2.2),(3.10)$ is automatically satisfied for all $\xi$ if it is satisfied in one point, i.e. if (for instance) (3.11) holds.

Lemma 3.6 Let $\lambda \in \mathbb{C}_{r}$ (1.8). Then, there exists a 2-dimensional subspace of solutions $\Phi^{s}(\xi ; \lambda)=\operatorname{span}\left\{\phi_{1}^{s}(\xi ; \lambda), \phi_{2}^{s}(\xi ; \lambda)\right\}$ of (2.2) that is invariant under translations by $2 L$, where the solutions $\phi_{1,2}^{s}(\xi ; \lambda)$ satisfy the following properties.

(i) There exists a positive $\mathcal{O}(1)$ number $C=C(\lambda)$ such that

$$
\begin{aligned}
& \phi_{1}^{s}(\xi ; \lambda, \varepsilon)=E_{+}^{s}(\lambda, \varepsilon) e^{\Lambda^{s} \xi}+\mathcal{O}\left(e^{-C|\xi|}\right) \\
& \phi_{2}^{s}(\xi ; \lambda, \varepsilon)=E_{-}^{s}(\lambda, \varepsilon) e^{-\Lambda^{s} \xi}+\mathcal{O}\left(e^{-C|\xi|}\right),
\end{aligned}
$$

for $\xi \in \mathcal{I}_{-}$. 
(ii) There exists an $\mathcal{O}(1)$ number $C_{\mathcal{P}}=C_{\mathcal{P}}(\lambda)$, such that $\left\|\phi_{1,2}^{s}(\xi)\right\| \leq C_{\mathcal{P}}$ and $u_{1,2}^{s}(\xi)=$ $1+\mathcal{O}(\varepsilon)$ for $\xi \in \mathcal{P}_{0}$.

(iii) There exist transmission functions $t_{i j}=t_{i j}(\lambda, \varepsilon)$ and a positive $\mathcal{O}(1)$ number $C=C(\lambda)$, such that

$$
\begin{array}{llll}
\phi_{1}^{s}(\xi ; \lambda, \varepsilon) & =t_{11} E_{+}^{s}(\lambda, \varepsilon) e^{\Lambda^{s} \xi} & +t_{21} E_{-}^{s}(\lambda, \varepsilon) e^{-\Lambda^{s} \xi} & +\mathcal{O}\left(e^{-C \xi}\right) \\
\phi_{2}^{s}(\xi ; \lambda, \varepsilon)=t_{12} E_{+}^{s}(\lambda, \varepsilon) e^{\Lambda^{s} \xi} & +t_{22} E_{-}^{s}(\lambda, \varepsilon) e^{-\Lambda^{s} \xi} & +\mathcal{O}\left(e^{-C \xi}\right)
\end{array}
$$

for $\xi \in \mathcal{I}_{+}$.

The subspace $\Phi^{s}(\xi ; \lambda)$ is uniquely determined by (3.10) and properties (i),(ii) and (iii). Furthermore, the subspace $\Phi^{s}(\xi)$ is closed under reflection in $\xi=0$, i.e. if $\phi^{s}(\xi) \in \Phi^{s}(\xi ; \lambda)$, then $R \phi^{s}(-\xi) \in \Phi^{s}(\xi ; \lambda)$.

Note that the coefficients $m_{i j}$ in (3.11) and the $t_{i j}$ in (3.13) are at leading order related by

$$
M^{s}(\lambda, \varepsilon)=\left(\begin{array}{cc}
m_{11}(\lambda, \varepsilon) & m_{12}(\lambda, \varepsilon) \\
m_{21}(\lambda, \varepsilon) & m_{22}(\lambda, \varepsilon)
\end{array}\right)=\left(\begin{array}{cc}
t_{11}(\lambda, \varepsilon) / \Delta^{2} & t_{12}(\lambda, \varepsilon) / \Delta^{2} \\
\Delta^{2} t_{21}(\lambda, \varepsilon) & \Delta^{2} t_{22}(\lambda, \varepsilon)
\end{array}\right),
$$

with $\Delta=\Delta\left(\Lambda^{s}\right)$ as in (1.11).

Proof. The proof consists of three parts. First, we prove that there exist two independent solutions to (2.2) that do not grow as the most unstable eigenmode $\left(E_{+}^{f} e^{\Lambda^{f}|\xi|}\right)$ on both outer regions $\mathcal{I}_{-}$and $\mathcal{I}_{+}$. Then, we prove that these solutions satisfy the properties in the Lemma. Finally, we show that these solutions can be perturbed to span a subspace that satisfies (3.10).

Define $\Sigma_{-}(\xi ; \lambda)$ as the linear 3-dimensional space of solutions of $(2.2)$, spanned by the solutions that satisfy the three boundary conditions $\phi(-L)=E_{ \pm}^{s}, E_{+}^{f}$ and $\Sigma_{+}(\xi ; \lambda)$ as the 3 -dimensional family of solutions, spanned by the solutions that satisfy the three boundary conditions $\phi(+L)=E_{ \pm}^{s}, E_{-}^{f}$. It follows from a dimension count that the intersection $\Sigma_{-}(\xi ; \lambda) \cap \Sigma_{+}(\xi ; \lambda)$ exists and is at least 2-dimensional. Furthermore, $\phi_{1}^{f}(\xi)$ is contained in $\Sigma_{-}(\xi ; \lambda)$ by construction, but it is not part of $\Sigma_{+}(\xi ; \lambda)$, since $t^{f}(\lambda) \neq 0$ on $\mathbb{C}_{r}$ (Lemma 3.5). Therefore $\Sigma_{-}(\xi ; \lambda) \cap \Sigma_{+}(\xi ; \lambda)$ is exactly 2-dimensional.

The solutions in $\Sigma_{-} \cap \Sigma_{+}$are spanned by two solutions $\tilde{\phi}_{1,2}(\xi)$, that satisfy the properties (i) - (iii) in the Lemma. In particular, we have

$$
\begin{aligned}
& \tilde{\phi}_{1}(\xi ; \lambda, \varepsilon)=E_{+}^{s}(\lambda, \varepsilon) e^{\Lambda^{s} \xi} \quad+\mathcal{O}\left(e^{-C|\xi|}\right) \\
& \tilde{\phi}_{2}(\xi ; \lambda, \varepsilon)=E_{-}^{s}(\lambda, \varepsilon) e^{-\Lambda^{s} \xi} \\
& +\mathcal{O}\left(e^{-C|\xi|}\right),
\end{aligned}
$$

for $\xi \in \mathcal{I}_{-}$and for some (well defined) positive $\mathcal{O}(1)$ number $C$ and there exist transmission functions $\tilde{t}_{11}$ and $\tilde{t}_{21}$, such that

$$
\begin{aligned}
& \tilde{\phi}_{1}(\xi)=\tilde{t}_{11} E_{+}^{s}(\lambda, \varepsilon) e^{\Lambda^{s} \xi}+\tilde{t}_{21} E_{-}^{s}(\lambda, \varepsilon) e^{-\Lambda^{s} \xi}+\mathcal{O}\left(e^{-C \xi}\right) \\
& \tilde{\phi}_{2}(\xi)=\tilde{t}_{12} E_{+}^{s}(\lambda, \varepsilon) e^{\Lambda^{s} \xi}+\tilde{t}_{22} E_{-}^{s}(\lambda, \varepsilon) e^{-\Lambda^{s} \xi}+\mathcal{O}\left(e^{-C \xi}\right)
\end{aligned}
$$

for $\xi \in \mathcal{I}_{+}$. The proof of the existence of a solution $\tilde{\phi}_{1}(\xi)$ of $(2.2)$ with these properties is essentially the same as the combined proofs of Lemmas 4.4-4.7 in [4]. Though the proofs given in [4], apply to the study of an eigenvalue problem for the linearization around a homoclinic solution, the proofs carry over to the periodic eigenvalue problem in this paper. The proofs in [4] are by contradiction, showing that a solution that does not satisfy (3.15) in $\mathcal{I}_{-}$must grow as $E_{+}^{f} e^{\Lambda^{f} \xi}$ in $\mathcal{I}_{+}$and is obviously not in $\Sigma_{+}-$see [4] for a full proof. To prove the existence of $\tilde{\phi}_{2}$, we consider $\phi^{*}(\xi)=R \tilde{\phi}_{1}(\xi)$, which by (3.16) is given by

$$
\phi^{*}(\xi)=\tilde{t}_{21} E_{+}^{s}(\lambda, \varepsilon) e^{\Lambda^{s} \xi}+\tilde{t}_{11} E_{-}^{s}(\lambda, \varepsilon) e^{-\Lambda^{s} \xi}+\mathcal{O}\left(e^{-C|\xi|}\right)
$$


for $\xi \in \mathcal{I}_{-}$. Thus, $\tilde{\phi}_{2}$ can be constructed as a linear combination of $\tilde{\phi}_{1}$ and $\phi^{*}$.

The solutions $\tilde{\phi}_{1,2}$ do not satisfy $(3.11)$ exactly, but since $\tilde{\phi}_{1,2} \in \Sigma_{-} \cap \Sigma_{+}$, there exists coefficients $\tilde{m}_{i j}, i, j=1,2$ such that

$$
\begin{aligned}
& \tilde{\phi}_{1}(L)=\tilde{m}_{11} \tilde{\phi}_{1}(-L)+\tilde{m}_{21} \tilde{\phi}_{2}(-L)+\mathcal{O}\left(e^{-C L}\right) \\
& \tilde{\phi}_{2}(L)=\tilde{m}_{12} \tilde{\phi}_{1}(-L)+\tilde{m}_{22} \tilde{\phi}_{2}(-L)+\mathcal{O}\left(e^{-C L}\right)
\end{aligned}
$$

where the $\mathcal{O}\left(e^{-C L}\right)$ terms are spanned by $E_{+}^{f}$ and $E_{-}^{f}$. Thus, the solutions $\tilde{\phi}_{1,2}(\xi)(3.15)$ are exponentially close to the desired solutions $\phi_{1,2}^{s}$ that satisfy (3.11), and thus (3.10), exactly. We adapt $\tilde{\phi}_{1,2}(\xi)$ by

$$
\begin{aligned}
& \phi_{1}^{s}(\xi)=\tilde{\phi}_{1}(\xi)+e^{-\left(C+\Lambda^{f}\right) L}\left(a_{11} \phi_{1}^{f}(\xi)+a_{21} \phi_{2}^{f}(\xi)\right) \\
& \phi_{2}^{s}(\xi)=\tilde{\phi}_{2}(\xi)+e^{-\left(C+\Lambda^{f}\right) L}\left(a_{12} \phi_{1}^{f}(\xi)+a_{22} \phi_{2}^{f}(\xi)\right),
\end{aligned}
$$

where $a_{i j}, i, j=1,2$ are free $\mathcal{O}(1)$ constants. Note that the correction terms are at most $\mathcal{O}\left(e^{-C L}\right)$, Lemma 3.2. Using the structure of $\phi_{1,2}^{f}( \pm L)$, the solutions $\phi_{1,2}^{s}(\xi)$ can now be made to satisfy (3.11) exactly, by making $\mathcal{O}\left(e^{-C L}\right)$ corrections to the $\tilde{m}_{i j}$ 's - thereby defining the $m_{i j}$ 's - and choosing the $a_{i j}$ 's such that there are no rest terms spanned by $E_{+}^{f}$ and $E_{-}^{f}$. This straightforward linear algebra exercise determines the $a_{i j}$ 's, and thus the $\phi_{1,2}^{s}(\xi)$ 's, uniquely.

The observation that $\Phi^{s}(\xi)$ is closed under reflection in $\xi=0$ follows immediately from the uniqueness of $\Phi^{s}(\xi)$. Suppose, that there exists a solution $\phi^{s}(\xi) \in \Phi^{s}(\xi)$, such that $R \phi^{s}(-\xi) \notin \Phi^{s}(\xi)$ and let $\phi^{*}$ be an independent element of $\Phi^{s}$. Then, the subspace $\Phi^{*}(\xi)=$ $\operatorname{span}\left\{R \phi^{s}(-\xi), R \phi^{*}(-\xi)\right\}$ is a second subspace of solutions that satisfies the properties in the Lemma and that is invariant under translations by $2 L$. This contradicts the fact that $\Phi^{s}$ is determined uniquely by the properties in the Lemma and (3.10).

Remark 3.7 In the homoclinic limit $L \rightarrow \infty$, the slow solution $\phi_{1}^{s}(\xi)$ converges on $[-L, L]$ by construction to the unique solution of the stability problem $(3.3)$ associated to $\left(U_{h}(\xi), V_{h}(\xi)\right)=$ $\left(U_{p}(\xi ; \infty), V_{p}(\xi ; \infty)\right)$, that satisfies

$$
\lim _{\xi \rightarrow-\infty} \phi_{1}^{s}(\xi) e^{-\Lambda^{s} \xi}=E_{+}^{s} \text { and } \lim _{\xi \rightarrow \infty} \phi_{1}^{s}(\xi) e^{-\Lambda^{f} \xi}=(0,0,0,0)^{t}
$$

(Theorem 1.7, Remark 3.1, [3]). Furthermore, since $A_{p}(\xi ; \lambda)$ converges to $A_{h}(\xi ; \lambda)$ uniformly on $[-L, L]$ (Remark 3.1), the transmission functions $t_{i j}(\lambda)$ converge to a homoclinic limit $t_{i j}^{h}(\lambda)$ as $L \rightarrow \infty$. In this limit, the slow solution $\phi_{1}^{s}(\xi)$ satisfies (by construction),

$$
\lim _{\xi \rightarrow \infty} \phi_{1}^{s}(\xi) e^{-\Lambda^{s} \xi}=t_{11}^{h}(\lambda) E_{+}^{s}
$$

so that $\phi_{1}^{s}(\xi)$ is an eigenfunction of (3.3) if and only if $t_{11}^{h}(\lambda)=0$. Note, that the notation used here is different from the notation used in [3], $\phi_{1}^{s}(\xi)$ and $t_{11}^{h}(\lambda)$ are denoted by $\phi_{2}(\xi)$ and $t_{2}(\lambda)$ in [3]. It has been shown in [3] that all (non-trivial) eigenvalues $\lambda_{h}(\neq 0)$ of $(3.3)$ correspond to zeroes of $t_{2}(\lambda)$, i.e. of $t_{11}^{h}(\lambda)$.

\subsection{The decomposition of the Evans function}

We consider the vectors

$$
\begin{aligned}
& \mathbf{v}_{1}^{f}(\lambda)=\phi_{1}^{f}(-L ; \lambda)=e^{-\Lambda^{f} L} E_{+}^{f} \\
& \mathbf{v}_{2}^{f}(\lambda)=\phi_{2}^{f}(-L ; \lambda)=t^{f}(\lambda) e^{+\Lambda^{f} L} E_{-}^{f} \quad+\mathcal{O}\left(e^{\left(\Lambda^{f}-C\right) L}\right) \\
& \mathbf{v}_{1}^{s}(\lambda)=\phi_{1}^{s}(-L ; \lambda)=e^{-\Lambda^{s} L} E_{+}^{s}+\mathcal{O}\left(e^{-C L}\right) \\
& \mathbf{v}_{2}^{s}(\lambda)=\phi_{2}^{s}(-L ; \lambda)=e^{+\Lambda^{s} L} E_{-}^{s}+\mathcal{O}\left(e^{-C L}\right)
\end{aligned}
$$


and express the monodromy matrix $M_{L}(\lambda, \gamma)$ with respect to $V=\left\{\mathbf{v}_{1}^{f}, \mathbf{v}_{2}^{f}, \mathbf{v}_{1}^{s}, \mathbf{v}_{2}^{s}\right\}$. Since $\phi_{1}^{s}$ and $\phi_{2}^{s}(\xi)$ satisfy (3.10) and (3.11), the monodromy matrix has the form

$$
M_{L}(\lambda)=\left(\begin{array}{cc}
M^{f}(\lambda) & 0 \\
B(\lambda) & M^{s}(\lambda)
\end{array}\right),
$$

where each entry denotes a $2 \times 2$ matrix. The matrix $M^{s}$ is defined in $(3.14), M^{f}$ is - by construction - at leading order equal to $M_{L}^{r}(3.9)$ with $t_{p}^{r}(\lambda)$ replaced by $t^{f}(\lambda)$ - Lemma 3.5. The eigenvalues of $M_{L}(\lambda)$ (3.18) are independent of the coefficients in the block $B(\lambda)$, so that the Evans function can be decomposed

$$
\mathcal{D}(\lambda, \gamma ; L)=\operatorname{det}\left[M^{s}(\lambda)-\gamma I_{2}\right] \operatorname{det}\left[M^{f}(\lambda)-\gamma I_{2}\right] \equiv \mathcal{D}^{s}(\lambda, \gamma ; L) \mathcal{D}^{f}(\lambda, \gamma ; L) .
$$

Proposition 3.8 For all $\lambda \in \mathbb{C}_{r}$ (1.8) and $\gamma \in \mathbf{S}^{1}$, the Evans function $\mathcal{D}(\lambda, \gamma)$ associated to (2.2) can be decomposed into a product of a fast and a slow component, $\mathcal{D}^{f}(\lambda, \gamma)$ and $\mathcal{D}^{s}(\lambda, \gamma)$, that are given by

$$
\begin{aligned}
& \mathcal{D}^{f}(\lambda, \gamma ; L)=-\gamma t^{f}(\lambda) e^{2 \Lambda^{f} L}(1+\mathcal{O}(\varepsilon)) \\
& \mathcal{D}^{s}(\lambda, \gamma ; L)=\gamma^{2}-\left(\frac{1}{\Delta^{2}} t_{11}(\lambda, 0)+\Delta^{2} t_{22}(\lambda, 0)+\mathcal{O}(\varepsilon)\right) \gamma+1
\end{aligned}
$$

with $\Delta=\Delta(\lambda, L)$ as in (1.11). The fast Evans function $\mathcal{D}^{f}(\lambda, \gamma)$ is non-zero in $\mathbb{C}_{r}$ so that all $\gamma$-eigenvalues $\lambda=\lambda(\gamma, \varepsilon)$ of (2.2) in $\mathbb{C}_{r}$ must be zeroes of the slow Evans function $\mathcal{D}^{s}(\lambda, \gamma)$; $\lambda=\lambda(\gamma, \varepsilon)=\lambda(\gamma, 0)+\mathcal{O}(\varepsilon)$ is a $\gamma$-eigenvalue if $\lambda(\gamma, 0)$ solves

$$
\operatorname{Tr}\left(M^{s}(\lambda)\right)=\frac{1}{\Delta^{2}} t_{11}(\lambda, 0)+\Delta^{2} t_{22}(\lambda, 0) \in[-2,2]
$$

with $\gamma, \bar{\gamma} \in \mathbf{S}^{1}$ determined by $\operatorname{Tr}\left(M^{s}(\lambda)\right)=2 \operatorname{Re}[\gamma]$.

The $\mathcal{O}(\varepsilon)$ corrections in (3.19) are clearly uniform in $\gamma \in \mathbf{S}^{1}$ and $L=\mathcal{O}\left(1 / \varepsilon^{2}\right)$, but we cannot yet conclude that they are uniform in $\lambda$ (for $\lambda \in \mathbb{C}_{r}$ ), since we need more insight in the structure of $t_{11}(\lambda, \varepsilon)$ and $t_{22}(\lambda, \varepsilon)$ - see Section 4.2.

Proof. Due to the structure of $M^{f}(\lambda), \gamma \in \mathbf{S}^{1}$ does only appear linearly in the leading order expression of $D^{f}(\lambda, \gamma)$ for $\gamma \in \mathbb{C}_{r}$. Since $t^{f}(\lambda)$ is bounded away from zero in $\mathbb{C}_{r}$ (Lemma 3.5), it follows that $\mathcal{D}^{f}(\lambda, \gamma) \neq 0$ in $\mathbb{C}_{r}$. The determinant of $M^{s}(\lambda)-\gamma I_{2}$ can be computed using (3.14). Since $\Phi^{s}(\xi ; \lambda)$ is symmetric under reflection in $\xi=0$ (Lemma 3.6), it follows that the eigenvalues of $M^{s}(\lambda, \varepsilon)$ come in pairs, $\rho^{s}(\varepsilon)$ and $1 / \rho^{s}(\varepsilon)$. Hence, $\operatorname{det}\left[M^{s}(\lambda, \varepsilon)\right] \equiv 1$, so that (3.19) follows. The magnitude of the leading corrections in (3.19) follow in a straightforward fashion. Furthermore,

$$
\operatorname{Tr}\left(M^{s}(\lambda, 0)\right)=\frac{1}{\Delta^{2}} t_{11}(\lambda, 0)+\Delta^{2} t_{22}(\lambda, 0)=\rho^{s}(0)+\frac{1}{\rho^{s}(0)} .
$$

The observation that $\rho^{s}+1 / \rho^{s} \in[-2,2]$ if and only if $\rho^{s}=\gamma \in \mathbf{S}^{1}$, implies (3.20).

Remark 3.9 Since the eigenvalues of $M_{L}(\lambda)$ come in pairs, $\rho_{1,2}$ and $1 / \rho_{1,2}$, and since one of these pairs is formed by the eigenvalues of $M^{s}(\lambda), \rho^{s}$ and $1 / \rho^{s}$, it follows that the eigenvalues of $M^{f}(\lambda)$ also form a pair, $\rho^{f}$ and $1 / \rho^{f}$. The approach of Section 3.1 yields that $\rho^{f}=t^{f}(\lambda) e^{2 \Lambda^{f} L}$ at leading order (for $\lambda \in \mathbb{C}_{r}$ ).

\section{The $\mathcal{O}(1) \gamma$-eigenvalues}

In this section we determine the coefficients of the slow monodromy matrix $M^{s}(\lambda)(3.14)$ for $\lambda \in \mathbb{C}_{r}$, which yields by Proposition 3.8 explicit expressions for the $\gamma$-eigenvalues of equation (2.2). Furthermore, the possible existence of $\gamma$-eigenvalues near the reduced eigenvalues $\lambda_{j}^{r}$, i.e. $\lambda \notin \mathbb{C}_{r}$, is considered. 


\subsection{The jump $\mathcal{J}(\lambda, \varepsilon)$}

As in $[3,4]$, we determine $t_{11}$ and $t_{22}$ by relating the expressions for the slow solutions $\phi_{1,2}^{s}$ in the outer regions $\mathcal{I}_{-}$and $\mathcal{I}_{+}$to over-all properties of $\phi_{1,2}^{s}$ over the pulse region $\mathcal{P}_{0}$. We denote the value of $\phi_{1,2}^{s}$ on the boundaries of $\mathcal{P}_{0}$ by $\phi_{i,-}^{s}=\phi_{i}^{s}\left(-\frac{1}{\sqrt{\varepsilon}}\right)$ and $\phi_{i,+}^{s}=\phi_{i}^{s}\left(\frac{1}{\sqrt{\varepsilon}}\right)$. Thus by (3.2), the $v$ and $q$ components of $\phi_{i, \pm}^{s}$ are exponentially small (i.e. $\mathcal{O}\left(e^{-C / \sqrt{\varepsilon}}\right)$ ) and the leading order parts of the $u$ and $p$ components of $\phi_{i, \pm}^{s}$ are given by

$$
\begin{array}{ll}
u_{1,-}^{s}=1, & u_{1,+}^{s}=t_{11}+t_{21} \\
u_{2,-}^{s}=1, & u_{2,+}^{s}=t_{21}+t_{22} ; \\
p_{1,-}^{s}=\Lambda^{s}, & p_{1,+}^{s}=\Lambda^{s}\left(t_{11}-t_{21}\right) \\
p_{2,-}^{s}=-\Lambda^{s}, & p_{2,+}^{s}=\Lambda^{s}\left(t_{12}-t_{22}\right)
\end{array}
$$

Note that the expected difference $\Delta_{s} p_{i}^{s}=p_{i,+}^{s}-p_{i,-}^{s}$ between the right and left boundary of $\mathcal{P}_{0}$ is proportional to $\Lambda^{s}=\mathcal{O}\left(\varepsilon^{2}\right)$.

The two outer regions are connected to each other by the pulse region $\mathcal{P}_{0}$ where the fast components are $\mathcal{O}(1)$. We know from Lemma 3.6(ii) that the $u$-component of $\phi_{1,2}^{s}$ is to leading order constant over the pulse region. On the other hand, since both $u_{i}^{s}$ and $v_{i}^{s}$ are $\mathcal{O}(1)$ over the pulse region, the first equation of (2.1) tells us that $u_{\xi \xi}=\mathcal{O}\left(\varepsilon^{2}\right)$ on the pulse region. Therefore, there is a change in $p$ over the pulse region given by $\Delta_{\mathcal{P}} p_{i}^{s}=\int_{\mathcal{P}_{0}} u_{i, \xi \xi}^{s} d \xi=\mathcal{O}\left(\varepsilon^{2}\right)$, which is of the same order as the expected difference $\Delta_{s} p_{i}^{s}$. This yields the leading order matching conditions

$$
\begin{array}{lll}
u_{1,+}^{s}=u_{1,-}^{s} & , \quad t_{11}+t_{21}=1 ; \\
\Delta_{s} p_{1}^{s}=\Delta_{\mathcal{P}} p_{1}^{s} & , \quad \Lambda^{s}\left(t_{11}-t_{21}\right)=\Lambda^{s}+\Delta_{\mathcal{P}} p_{1}^{s} \\
u_{2,+}^{s}=u_{2,-}^{s} & , \quad t_{21}+t_{22}=1 ; \\
\Delta_{s} p_{2}^{s}=\Delta_{\mathcal{P}} p_{2}^{s} & , \quad \Lambda^{s}\left(t_{12}-t_{22}\right)=\Lambda^{s}+\Delta_{\mathcal{P}} p_{2}^{s}
\end{array}
$$

where the expressions in the second column follow from those in the first by substitution of the leading order parts of $u_{i, \pm}^{s}$ and $p_{i, \pm}^{s}$ (4.1). Thus, the transmission functions $t_{i j}$ can be expressed in terms of the jumps in the derivative $\Delta_{\mathcal{P}} p_{1,2}^{s}$.

In general, i.e for general solutions $\phi^{s} \in \Phi^{s}$ (Lemma 3.6), the jump in $p^{s}$ over the fast field $\mathcal{P}_{0}$ is given by

$$
\Delta_{\mathcal{P}} p^{s}=\mathcal{J}(\lambda, \varepsilon) u_{0}^{s}+\text { h.o.t. }
$$

where $u_{i}^{s}$ is the (to leading order constant) value of $u^{s}$ in $\mathcal{P}_{0}$. The function $\mathcal{J}(\lambda, \varepsilon)$ denotes a unit jump in the derivative, i.e. the jump in the derivative if $u_{0}^{s}=1$. The change in $p$ is found by integrating $u_{i, \xi \xi}^{s}$ over the pulse region,

$$
\Delta_{\mathcal{P}} p^{s}=\int_{\mathcal{P}_{0}} u_{\xi \xi}^{s} d \xi=-\varepsilon^{2} \int_{\mathcal{P}_{0}} \alpha_{1} U_{p}^{\alpha_{1}-1} V_{p}^{\beta_{2}} u_{0}^{s}+\beta_{1} U_{p}^{\alpha_{1}} V_{p}^{\beta_{2}-1} v^{s} d \xi+\text { h.o.t. }
$$

where we have replaced $u^{s}(\xi)$ by its leading order value $u_{0}^{s}$. We also substitute $u_{0}^{s}$ in the second equation of (2.1), which implies that $v_{i}^{s}$ satisfies at leading order

$$
v_{\xi \xi}+\left[\beta_{2} U_{p}^{\alpha_{2}}\left(V_{p}(\xi)\right)^{\beta_{2}-1}-(1+\lambda)\right] v=-\alpha_{2} U_{p}^{\alpha_{2}-1}\left(V_{p}(\xi)\right)^{\beta_{2}} u_{0}^{s} .
$$

We see that the solution $v^{s}$ is proportional to $u_{0}^{s}$, so that we can scale out this constant by writing $v^{s}=u_{0}^{s} \hat{v}^{s}$,

$$
\mathcal{J}(\lambda, \varepsilon)=\frac{\Delta_{\mathcal{P}} p^{s}}{u_{0}^{s}}+\text { h.o.t. }=-\varepsilon^{2} \int_{\mathcal{P}} \alpha_{1} U_{p}^{\alpha_{1}-1} V_{p}^{\beta_{2}}+\beta_{1} U_{p}^{\alpha_{1}} V_{p}^{\beta_{2}-1} \hat{v}^{s} d \xi+\text { h.o.t. }
$$

We now return to the evaluation of the transmission functions and thus set $u_{0}^{s}=1$ and $\hat{v}^{s}=v_{i}^{s}$.

Equation (4.5) is defined only for $\xi \in \mathcal{P}_{0}$ and we a priori need two boundary conditions to determine the exact solution $v_{i}^{s}$. It is a non-trivial procedure to obtain the exact boundary 
conditions. Since $\phi_{1}^{s}$ and $\phi_{2}^{s}$ span a family of solutions that is invariant under translations by $2 L$, there are two conditions that relate $\phi_{1,2}^{s}(\xi)$ to $\phi_{1,2}^{s}(\xi+2 L)$. However these conditions concern both the $u$ and $v$-components of $\phi_{1,2}^{s}$. In particular this means that the boundary conditions (on the boundaries of $\mathcal{P}_{0}$ ) on $v_{i}^{s}$ can only be formulated if the values of $u_{1,2}^{s}(L)$ are known. Fortunately, the exact boundary conditions are not necessary to determine a leading order expression for $v_{i}^{s}$. To show this we approximate (4.5) by a problem that is defined for $\xi \in \mathbb{R}$. It was noted in the existence analysis in Section 1.1 that the periodic solution $\left(U_{p}(\xi), V_{p}(\xi)\right)$ is exponentially close for $\xi \in[-L, L]$ to the solution $\left(U_{\mathcal{M}}(\xi), V_{\mathcal{M}}(\xi)\right)$ that is homoclinic to $\mathcal{M}$ (1.13) [6]. Hence the restriction to $\mathcal{P}_{0}$ of a solution defined on $\mathbb{R}$ to

$$
v_{\xi \xi}+\left[\beta_{2} U_{\mathcal{M}}^{\alpha_{2}}\left(V_{\mathcal{M}}(\xi)\right)^{\beta_{2}-1}-(1+\lambda)\right] v=-\alpha_{2} U_{\mathcal{M}}^{\alpha_{2}-1}\left(V_{\mathcal{M}}(\xi)\right)^{\beta_{2}}
$$

satisfies (4.5) up to exponentially small errors. Note that this expression is obtained from (4.5) by replacing $U_{p}$ and $V_{p}$ by $U_{\mathcal{M}}$ and $V_{\mathcal{M}}$ and setting $u_{0}^{s}=1$, or alternatively by direct linearization around $\left(U_{\mathcal{M}}, V_{\mathcal{M}}\right)$. The solution $v_{i}^{s}(\xi)$ is known and exponentially small at the boundaries of the pulse region $((3.12),(3.13))$. We may therefore conclude that the solution $v_{i}^{s}$ to (4.5) must be exponentially close to a bounded solution $v_{i n}$ of (4.6), independent of the precise boundary conditions.

Since (4.6) is a Sturm-Liouville problem, there exists a unique bounded solution $v_{i n}(\xi)$ to (4.6) if $\lambda \in \mathbb{C}_{r}$. Therefore, the change 'unit jump' in $p$ is in terms of $v_{i n}(\xi), U_{\mathcal{M}}$, and $V_{\mathcal{M}}$ given by

$$
\mathcal{J}(\lambda, \varepsilon)=-\varepsilon^{2} \int_{-\infty}^{\infty}\left[\alpha_{1} U_{\mathcal{M}}^{\alpha_{1}-1}\left(V_{\mathcal{M}}(\xi)\right)^{\beta_{1}}+\beta_{1} U_{\mathcal{M}}^{\alpha_{1}}\left(V_{\mathcal{M}}(\xi)\right)^{\beta_{1}-1} v_{i n}(\xi)\right] d \xi+\text { h.o.t. }
$$

Note that we have replaced the integration over the pulse region by an integration over $\mathbb{R}$, since the contributions of the integrals outside $\mathcal{P}_{0}$ are higher order terms $\left(V_{\mathcal{M}}\right.$ decays exponentially fast). To simplify this expression we approximate $U_{\mathcal{M}}$ and $V_{\mathcal{M}}$ by

$$
U_{\mathcal{M}}(\xi)=U_{p}(0) \text { and } V_{\mathcal{M}}(\xi)=\left(U_{p}(0)\right)^{-\alpha_{2} /\left(\beta_{2}-1\right)} w_{h}(\xi)
$$

and scale the fast component by setting

$$
v_{i n}(\xi)=-\alpha_{2}\left(U_{p}(0)\right)^{-1-\alpha_{2} /\left(\beta_{2}-1\right)} w_{i n}(\xi) .
$$

so that equation (4.6) scales into

$$
\left(\mathcal{L}^{r}-\lambda\right) w=w_{\xi \xi}+\left[\beta_{2}\left(w_{h}(\xi)\right)^{\beta_{2}-1}-(1+\lambda)\right] w=\left(w_{h}(\xi)\right)^{\beta_{2}},
$$

where $\mathcal{L}^{r}$ is the operator of the reduced linear eigenvalue problem (3.4). Note that this is exactly the same inhomogeneous problem that was derived in the study of the eigenvalues of the linearization around the homoclinic pulse solution in [3]. In terms of the bounded solution $w_{\text {in }}$ to $(4.7)$, the leading order part of $\mathcal{J}(\lambda, \varepsilon)$ is given by

$$
\mathcal{J}(\lambda, \varepsilon)=-\varepsilon^{2} W\left(\beta_{1}, \beta_{2}\right) U_{0}^{D /\left(\beta_{2}-1\right)}\left[\alpha_{1}-\alpha_{2} \beta_{1} \mathcal{R}(\lambda)\right]
$$

where $W\left(\beta_{1}, \beta_{2}\right)$ has been defined in $(1.22)$ and $\mathcal{R}(\lambda)$ is given by

$$
\mathcal{R}(\lambda)=\frac{1}{W\left(\beta_{1}, \beta_{2}\right)} \int_{-\infty}^{\infty} w_{i n}(\xi ; \lambda)\left(w_{h}(\xi)\right)^{\beta_{1}-1} d \xi
$$

Note that $\mathcal{R}(\lambda)$ corresponds to the $\mathcal{R}(\lambda)$ defined in [3], the only difference is the $1 / W\left(\beta_{1}, \beta_{2}\right)$ factor.

Lemma 4.1 For $\lambda \in \mathbb{C}_{r}$, the unit jump $\mathcal{J}(\lambda, \varepsilon)=\mathcal{J}(\lambda, L, \varepsilon)$ is given by

$$
\mathcal{J}(\lambda, L, \varepsilon)=-2 \varepsilon^{2} \sqrt{\mu} \tanh \left(\varepsilon^{2} \sqrt{\mu} L\right)\left[\alpha_{1}-\alpha_{2} \beta_{1} \mathcal{R}(\lambda)\right](1+\mathcal{O}(\varepsilon)),
$$

where the $\mathcal{O}(\varepsilon)$ correction is uniform in $\lambda \in \mathbb{C}_{r}$ and in $L=\mathcal{O}\left(1 / \varepsilon^{2}\right)$. 
Proof. The proof follows immediately from the above calculations (and (1.25)), while keeping track of the magnitude of the leading order correction terms. The observation that the approximation is uniform in $\lambda \in \mathbb{C}_{r}$ is based on the fact that the function $\mathcal{R}(\lambda)$ is uniformly bounded in $\mathbb{C}_{r}$, see [3].

The solution $w_{\text {in }}$ of (4.7) has been determined in terms of hypergeometric functions in [3]. We will not give all details of this calculation, instead the interested reader is referred to [3]. The transformation into hypergeometric function starts with the observation that $w_{i n}$ decays as $e^{-\sqrt{1+\lambda}|\xi|}$ as $\xi \rightarrow \pm \infty$. Therefore we search for solutions of the form

$$
w_{i n}(\xi)=C F(\xi)\left(w_{h}(\xi)\right)^{P}
$$

where $P=\sqrt{1+\lambda}, F(\xi)$ is the new unknown function and $C$ is a constant that is for convenience set to be

$$
C=\frac{\left[2\left(\beta_{2}+1\right)\right]^{\left(\beta_{2}-P\right) /\left(\beta_{2}-1\right)}}{\left(\beta_{2}-1\right)^{2}} .
$$

After the introduction of a new independent variable $z \in(0,1)$ by

$$
z=\frac{1}{2}\left(1-\frac{\dot{w}_{h}(\xi)}{w_{h}(\xi)}\right)
$$

the inhomogeneous equation (4.7) is transformed into the inhomogeneous hypergeometric differential equation $\left({ }^{\prime}=\partial / \partial z\right)$

$$
z(1-z) F^{\prime \prime}+[c-(a+b+1) z] F^{\prime}-a b F=[z(1-z)]^{(1-P) /\left(\beta_{2}-1\right)},
$$

with $a, b$ and $c$ given by

$$
a=\frac{2 P+2 \beta_{2}}{\beta_{2}-1}, \quad b=\frac{2 P-\beta_{2}-1}{\beta_{2}-1}, \quad c=\frac{2 P+\beta_{2}-1}{\beta_{2}-1} .
$$

The solution space of the homogeneous part of (4.11) is spanned by the hypergeometric functions

$$
H_{1}(z)=F_{1}^{2}(a, b|c| z) \text { and } H_{2}(z)=H_{1}(1-z)=F_{1}^{2}(a, b|c| 1-z) .
$$

Note that the symmetry around $z=\frac{1}{2}$ corresponds to the reversibility symmetry $\xi \leftrightarrow-\xi$ in (4.7). The bounded solution to the inhomogeneous problem can then be found using a classical variation of constants approach, see appendix B in [3] for the details. Using the solution $F(z)$ thus found, the expression for $\mathcal{R}(\lambda)$ can be written as

$$
\mathcal{R}(\lambda)=\frac{\hat{\mathcal{R}}(\lambda)}{\mathcal{B}\left(\beta_{1}, \beta_{2}\right)}=\frac{1}{\mathcal{B}\left(\beta_{1}, \beta_{2}\right)} \int_{0}^{1} F(z)[z(1-z)]^{\left(P+\beta_{1}-\beta_{2}\right) /\left(\beta_{2}-1\right)} d z,
$$

with

$$
\mathcal{B}\left(\beta_{1}, \beta_{2}\right)=\frac{\left(\beta_{2}-1\right)^{2}}{2\left(\beta_{2}+1\right)} \int_{0}^{1}[z(1-z)]^{\left(\beta_{1}-\beta_{2}+1\right) /\left(\beta_{2}-1\right)} d z .
$$

Apart from a constant, the function $\mathcal{B}\left(\beta_{1}, \beta_{2}\right)$ corresponds to $W\left(\beta_{1}, \beta_{2}\right)$ in the definition of $\mathcal{R}(\lambda)(4.9) ; \hat{\mathcal{R}}(\lambda)$ corresponds to $\mathcal{R}(\lambda)$ as defined in $[3]$.

Obviously, the function $\mathcal{R}(\lambda)$ is essential for the evaluation of (4.8) - [3] for more details. Therefore we give a few important characteristics of $\mathcal{R}(\lambda)$ that can be obtained without the transformation to hypergeometric functions. The inhomogeneous equation (4.7) does not necessarily have a bounded solution if $\lambda$ is equal to one of the eigenvalues $\lambda_{j}^{r}$ (3.6) of the operator $\mathcal{L}^{r}$. A bounded solution to (4.7) exists if and only if the corresponding eigenfunction $w_{j}^{r}(\xi)$ satisfies the solvability condition

$$
\int_{-\infty}^{+\infty}\left(w_{h}(\xi)\right)^{\beta_{2}} w_{j}^{r}(\xi) d \xi=0
$$


Since the eigenfunction $w_{j}^{r}$ corresponding to the eigenvalue $\lambda_{j}^{r}$ is even for $j$ even and since $w_{h}$ is even, the solvability condition is not satisfied for $j$ even. Hence for $\lambda=\lambda_{j}^{r}, j$ even, there exists no bounded solution to (4.7). The function $\mathcal{R}(\lambda)$ is meromorphic and has a simple pole at each of the even eigenvalues $\lambda_{2 k}^{r}$ (and nowhere else). On the other hand, the eigenfunctions $w_{2 k+1}^{r}$ are odd for the odd eigenvalues $\lambda_{2 k+1}^{r}$, so that the integrand in (4.13) is odd and the integral vanishes. Thus, for the odd eigenvalues there exists a bounded solution to (4.7), though it is not unique. Nevertheless, $\mathcal{R}(\lambda)$ is well-defined in $\lambda_{2 k+1}^{r}$ and smooth in a neighborhood of $\lambda_{2 k+1}^{r}$. In particular it is easy to verify that if $\lambda=\lambda_{1}^{r}=0,(4.7)$ is solved by

$$
w_{\text {in }}(\xi)=\frac{1}{\beta_{2}-1} w_{h}(\xi)+C \dot{w}_{h}(\xi)
$$

where $C$ may be any real number. Thus, (4.9) can be evaluated at $\lambda=0$,

$$
\mathcal{R}(0)=\frac{1}{W\left(\beta_{1}, \beta_{2}\right)} \frac{1}{\left(\beta_{2}-1\right)} \int_{-\infty}^{\infty} w_{h}^{\beta_{1}} d \xi=\frac{1}{\beta_{2}-1},
$$

since $\dot{w}_{h}$ is odd. We conclude by $(4.10),(1.3)$ that, at leading order,

$$
\mathcal{J}(0,0)=-2 \varepsilon^{2} \sqrt{\mu}\left(\frac{D}{\beta_{2}-1}+1\right) \tanh \left(\varepsilon^{2} \sqrt{\mu} L\right) .
$$

Remark 4.2 For general $\beta_{2}, \mathcal{R}(\lambda), \hat{\mathcal{R}}(\lambda)$ (4.12) is expressed in terms of (integrals over) hypergeometric function $F(z)$. However, $F(z)$ reduces to a product of polynomials of at most order $k$ if $\beta_{2}=\frac{k+1}{k-1}=3,2, \frac{5}{3}, \ldots(k=2,3, \ldots)$. For example, the classical Gierer-Meinhardt problem has $\beta_{2}=2$, so that $\mathcal{R}(\lambda)$ is based on cubic, quadratic and linear polynomials [3].

\subsection{Explicit expressions for the $\mathcal{O}(1)$ slow $\gamma$-eigenvalues}

The leading order expressions for the transmission functions $t_{i j}$ can be determined by solving the equations (4.2). By (4.3), this results in

$$
\begin{aligned}
& t_{11}(\lambda, \varepsilon)=1+\frac{\mathcal{J}(\lambda, \varepsilon)}{2 \Lambda^{s}(\lambda, \varepsilon)}+\mathcal{O}(\varepsilon), \quad t_{12}(\lambda, \varepsilon)= \frac{\mathcal{J}(\lambda, \varepsilon)}{2 \Lambda^{s}(\lambda, \varepsilon)}+\mathcal{O}(\varepsilon), \\
& t_{21}(\lambda, \varepsilon)=-\frac{\mathcal{J}(\lambda, \varepsilon)}{2 \Lambda^{s}(\lambda, \varepsilon)}+\mathcal{O}(\varepsilon), \quad t_{22}(\lambda, \varepsilon)=1-\frac{\mathcal{J}(\lambda, \varepsilon)}{2 \Lambda^{s}(\lambda, \varepsilon)}+\mathcal{O}(\varepsilon),
\end{aligned}
$$

with $\Lambda^{s}(\lambda, \varepsilon)=\varepsilon^{2} \sqrt{\mu+\lambda}(3.2)$. The leading order approximations $t_{i j}(\lambda, 0)$ of $t_{i j}(\lambda, \varepsilon)$ can all be expressed in terms of

$$
\frac{\mathcal{J}(\lambda, \varepsilon)}{2 \Lambda^{s}(\lambda, \varepsilon)}=-\frac{\sqrt{\mu}}{\sqrt{\mu+\lambda}} \tanh \left(\varepsilon^{2} \sqrt{\mu} L\right)\left[\alpha_{1}-\alpha_{2} \beta_{1} \mathcal{R}(\lambda)\right]+\mathcal{O}(\varepsilon),
$$

Lemma 4.1. Note that $\varepsilon^{2} L=\mathcal{O}(1)$ - Theorem 1.7 - so that $t_{i j}(\lambda, 0)$ indeed does not depend on $\varepsilon$. Moreover, we have found that $t_{i j}(\lambda, \varepsilon)$ is uniformly bounded as function of $\lambda$ for $\lambda \in \mathbb{C}_{r}$, so that we now also may conclude that the $\mathcal{O}(\varepsilon)$ corrections in (3.19) - Proposition 3.8 - are uniform in $\lambda\left(\lambda \in \mathbb{C}_{r}\right)$.

With these expressions and with (3.14), we have for $\lambda \in \mathbb{C}_{r}$ obtained a leading order expression for the 'slow' monodromy matrix,

$$
M^{s}(\lambda, 0)=\left(\begin{array}{cc}
\frac{1}{\Delta^{2}}\left(1+\frac{\mathcal{J}}{2 \Lambda^{s}}\right) & \frac{1}{\Delta^{2}} \frac{\mathcal{J}}{2 \Lambda^{s}} \\
-\Delta^{2} \frac{\mathcal{J}}{2 \Lambda^{s}} & \Delta^{2}\left(1-\frac{\mathcal{J}}{2 \Lambda^{s}}\right)
\end{array}\right)
$$


(1.11); $M^{s}(0,0)$ can be evaluated explicitly,

$$
M^{s}(0,0)=\left(\begin{array}{cc}
\frac{1}{\Delta_{0}^{2}}\left(1-\left(\frac{D}{\beta_{2}-1}+1\right) \tanh \left(\varepsilon^{2} \sqrt{\mu} L\right)\right) & -\frac{1}{\Delta_{0}^{2}}\left(\frac{D}{\beta_{2}-1}+1\right) \tanh \left(\varepsilon^{2} \sqrt{\mu} L\right) \\
\Delta_{0}^{2}\left(\frac{D}{\beta_{2}-1}+1\right) \tanh \left(\varepsilon^{2} \sqrt{\mu} L\right) & \Delta_{0}^{2}\left(1+\left(\frac{D}{\beta_{2}-1}+1\right) \tanh \left(\varepsilon^{2} \sqrt{\mu} L\right)\right)
\end{array}\right)
$$

(4.15), where $\Delta_{0}=\Delta(0, L)$ (1.11). Substitution of the expressions (4.16) and (4.17) in (3.20) gives the desired leading order expression for the position of the slow $\gamma$-eigenvalues (1.10) in Theorem 1.1 with, of course, $T^{s}(\lambda, L)=\operatorname{Tr}\left(M^{s}(\lambda, 0)\right)$.

The $\gamma$-eigenfunctions $\phi_{\gamma}^{s}$ corresponding to the $\mathcal{O}(1)$-eigenvalues, are of the form

$$
\phi_{\gamma}^{s}(\xi)=c_{1} \phi_{1}^{s}(\xi)+c_{2} \phi_{2}^{s}(\xi),
$$

where $\left(c_{1}, c_{2}\right)^{t}$ is the eigenvector of $M^{s}$ that corresponds to the eigenvalue $\gamma$ of $M^{s}$. Therefore, $\phi_{\gamma}^{s}$ has the same properties as $\phi_{1}^{s}$ and $\phi_{2}^{s}$. Of course, we have so far only paid attention to the stability problem restricted to one spatial period (centered around $\xi=0$ ). By construction, the $u$-component $u_{\gamma}^{s}$ of $\phi_{\gamma}^{s}$ is to leading order constant over any pulse region $\mathcal{P}_{n}$, i.e. the pulse region centered around $\xi=2 n L$, and is give by $u_{\gamma}^{s}=u_{\gamma}^{s}(0) \gamma^{n}$ with in principle $u_{\gamma}^{s}(0)=c_{1}+c_{2}$, however, $u_{\gamma}^{s}(0)$ can be set to be 1 (by choosing $c_{1,2}$ appropriately). Since the leading order part of the $v$-component $v_{\gamma}^{s}$ of $\phi_{\gamma}^{s}$ is a combination of the leading order parts of $v_{1}^{s}$ and $v_{2}^{s}$, the leading order part of $v_{\gamma}^{s}$ in a pulse region is a multiple of $v_{i n}$, the bounded solution to (4.6). Hence, the leading order part of $v_{\gamma}^{s}$ on $\mathbb{R}$ is given by the uniform approximation

$$
v_{\gamma}^{s}(\xi)=\sum_{n \in \mathbb{Z}} \gamma^{n} v_{i n}(\xi-2 n L)
$$

Note that $v_{\gamma}^{s}$ satisfies (4.5) - with $u_{0}^{s}=\gamma^{n}$ - on each pulse region $\mathcal{P}_{n}$, up to exponentially small errors.

\subsection{Near the reduced eigenvalues $\lambda_{j}^{r}$}

For $\lambda \in \mathbb{C}_{r}$ (1.8), the position of the $\gamma$-eigenvalues of (2.2) is determined by Proposition 3.8. To complete the picture, it is necessary to determine the (possible existence of) zeroes of $\mathcal{D}(\lambda, \gamma)$ for $\lambda$ near $\lambda_{j}^{r}$ (3.6), i.e. inside the balls $B\left(\lambda_{j}^{r}, \delta\right)(1.9)$. Since $\lambda_{0}^{r}>0$, the presence of a $\gamma$-eigenvalue in $B\left(\lambda_{0}^{r}, \delta\right)$ would imply the instability of the periodic pulse solution. Furthermore, $\lambda_{1}^{r}=0$, so that the $\gamma$-eigenvalues in $B\left(\lambda_{1}^{r}, \delta\right)$ can in principle also lie in the unstable half plane. Thus, the analysis of the eigenvalue problem near the eigenvalues of the reduced eigenvalue problem is crucial for the stability of the periodic pulse solutions.

After the introduction of the concept of a $\gamma$-eigenvalue and the monodromy matrix $M_{L}$, the analysis of the Evans function for periodic patterns has been remarkably similar to that of the Evans function for localized homoclinic patterns, as developed in $[3,4]$ - especially concerning the technical details of the construction of the fast and slow functions $\phi_{1,2}^{f, s}(\xi ; \lambda, \varepsilon)$. This is no longer the case for $\mathcal{D}(\lambda, \gamma)$ near the reduced eigenvalues $\lambda_{j}^{r}$, although the difference is rather subtle.

In the context of localized patterns, the Evans function can be decomposed into a product of two transmission functions, $t_{1}(\lambda)$ and $t_{2}(\lambda)$ ( $\times$ a constant), where $t_{1}(\lambda)$ corresponds to $t^{f}(\lambda)$ in the limit $L \rightarrow \infty$, and $t_{2}(\lambda)$ to $t_{11}(\lambda)$ (Remark 3.7). Using the analyticity of $t_{1}(\lambda)$, it is shown in [3] that $t_{1}(\lambda)$ must have a zero $\lambda_{j}^{*}$ near each of the reduced eigenvalues $\lambda_{j}^{r}$ (compare to Lemma 3.5)). However, this zero is not automatically a zero of the associated Evans function, since it is also shown in [3] that $t_{2}(\lambda)$ has a (simple) pole at $\lambda_{j}^{*}$ for $j$ even. This zero-pole cancellation phenomenon for even reduced eigenvalues is the resolution to the so-called 'NLEP paradox', which states that pulse solutions may be stable, although the fast component of the 
Evans function, $t_{1}(\lambda)$, has an $\mathcal{O}(1)$ unstable zero at $\lambda_{0}^{*}[3,4]$. The pole of $t_{2}(\lambda)$ corresponds directly to an obstruction to the construction of the slow solution $\phi_{2}(\xi)$, that corresponds to $\phi_{1}^{s}(\xi)$ here (Remark 3.7).

In the periodic setting, the homoclinic decomposition of the Evans function into a product of $t_{1}(\lambda)$ and $t_{2}(\lambda)$ corresponds to the decomposition $\mathcal{D}(\lambda, \gamma)=\mathcal{D}^{f}(\lambda, \gamma) \mathcal{D}^{s}(\lambda, \gamma)$ - Proposition 3.8. It follows from the proof of Lemma 3.6 that the construction of $\phi_{1}^{s}(\xi)$ also breaks down for those $\lambda$ for which $t^{f}(\lambda)=0$, since in that case $\phi_{1}^{f}(\xi) \in \Sigma_{-}(\xi ; \lambda) \cap \Sigma_{+}(\xi ; \lambda)$, so that the space $\Sigma_{-}(\xi ; \lambda) \cap \Sigma_{+}(\xi ; \lambda)$ cannot be spanned by two functions $\tilde{\phi}_{1}^{s}(\xi)$ and $\tilde{\phi}_{2}^{s}(\xi)$ that are both independent of $\phi_{1}^{f}(\xi)$ (which is necessary for the construction of the monodromy matrices $M^{f}(\lambda)$ and $M^{s}(\lambda)$ - Section 3.3). This is completely analogous to the homoclinic case. However, the fact that $t^{f}(\lambda)=0$ does not necessarily imply that there exists a zero of $\mathcal{D}^{f}(\lambda, \gamma), t^{f}(\lambda)$ is just one of the ingredients of the matrix $M^{f}(\lambda)$. Hence, there is no natural counterpart of $\lambda_{j}^{*}$ in the periodic case, so that there is no obvious 'NLEP paradox' in the periodic case (such a 'periodic NLEP paradox' would be based on the existence of zeroes of $\left.\mathcal{D}^{f}(\lambda, \gamma)\right)$. Likewise, there are no obvious poles for $\mathcal{D}^{s}(\lambda, \gamma)$.

Moreover, it is a priori not clear that either one of $\mathcal{D}^{f}(\lambda, \gamma)$ or $\mathcal{D}^{s}(\lambda, \gamma)$ is analytic near a reduced eigenvalue $\lambda_{j}^{r}$. The transmission function $t^{f}(\lambda)$ is analytic by Lemma 3.2 (a result that is completely analogous to that for $t_{1}(\lambda)$ in the homoclinic case). However, the matrices $M^{s}(\lambda)$ and $M^{f}(\lambda)$ are determined by the decomposition of the vectors $\phi_{1,2}^{f, s}(L ; \lambda)$ with respect to the $\operatorname{span}\left\{\mathbf{v}_{1,2}^{f, s}\right\}=\left\{\phi_{1,2}^{f, s}(-L ; \lambda)\right\}-$ Section 3.3. Since $\left\{\mathbf{v}_{1,2}^{f, s}\right\}$ is not orthogonal, all coefficients will in principle depend on all vectors $\mathbf{v}_{1,2}^{f, s}$. Due to the obstructions in the construction of $\phi_{1,2}^{s}(\xi ; \lambda)$ for $t^{f}(\lambda)=0$, it can a priori not be concluded that $\mathbf{v}_{1,2}^{s}$ depend analytically on $\lambda$ near a $\lambda_{j}^{r}$. As a consequence, it is a priori unclear whether the $\mathcal{D}^{f, s}(\lambda, \gamma)$ 's are analytic near $\lambda_{j}^{r}$.

Of course the fast and slow Evans functions $\mathcal{D}^{f, s}(\lambda, \gamma)$ are, by construction, analytic for $\lambda \in \mathbb{C}_{r}$. Thus, both functions must have analytic continuations into the balls $B\left(\lambda_{j}^{r}, \delta\right)(1.9)$. However, the interpretation of $\mathcal{D}^{f, s}(\lambda, \gamma)$ in terms of well-determined monodromy matrices $M^{f, s}(\lambda)$ (Section 3.3) becomes unclear near $\lambda_{j}^{r}$. For instance, it follows from a more detailed analysis (not given here) that the leading order approximations given in Lemma 3.6 obtain leading order corrections as $\lambda$ approaches $\lambda_{j}^{r}$. Therefore, we are forced to conclude that the decomposition of $\mathcal{D}(\lambda, \gamma)$ into a product of $\mathcal{D}^{f}(\lambda, \gamma)$ and $\mathcal{D}^{s}(\lambda, \gamma)$ does not give insight into the existence of $\gamma$-eigenvalues near $\lambda_{j}^{r}$. Nevertheless, we can use the decomposition for $\lambda \in \mathbb{C}_{r}$ and $\mathcal{O}(\delta)$ near $\lambda_{j}^{r}$, and apply a winding number argument.

Proposition 4.3 Let $0<\varepsilon \ll \delta \ll 1$ and $\mu \gg \delta,\left|\alpha_{2}\right| \gg \sqrt{\delta}$, and $\left|\alpha_{1}\right|=\mathcal{O}(1)$ with respect to $\delta$. Assume that the period $2 L$ of the fundamental periodic pattern $\left(U_{p}(\xi ; L), V_{p}(\xi ; L)\right)$ (Theorem 1.7) satisfies $\varepsilon^{2} L=\mathcal{O}(1)$ or $\varepsilon^{2} L \gg \mathcal{O}(1)$ with respect to $\delta$.

(i) If $j=2 k$ is even, then eigenvalue problem (2.2) has no $\gamma$-eigenvalues in the ball $B\left(\lambda_{2 k}^{r}, \delta\right)$. (ii) If $j=2 k+1$ is odd and if $\operatorname{Tr}\left(M^{s}\left(\lambda_{2 k+1}^{r}, 0\right)\right) \notin[-2,2]$, then for every $\gamma \in \mathbf{S}^{1}$, eigenvalue problem (2.2) has one (and only one) $\gamma$-eigenvalue $\lambda(\gamma) \in B\left(\lambda_{2 k+1}^{r}, \delta\right)$ (counting multiplicities), and $\lambda(\gamma) \in \mathbb{R}$; if $\operatorname{Tr}\left(M^{s}\left(\lambda_{2 k+1}^{r}, 0\right)\right) \in[-2,2]$ then (2.2) has one $\gamma$-eigenvalue $\lambda(\gamma) \in B\left(\lambda_{2 k+1}^{r}, \delta\right) \cap \mathbb{R}$ for any $\gamma \in \mathbf{S}^{1}$ with $\left|\operatorname{Re}[\gamma]-\frac{1}{2} \operatorname{Tr}\left(M^{s}\left(\lambda_{2 k+1}^{r}, 0\right)\right)\right| \gg \delta$.

Only the local $\gamma$-eigenvalues near $\lambda_{1}^{r}=0$ are relevant for the stability analysis. These small eigenvalues are studied in the forthcoming section, in this analysis both subcases of (ii) will be encountered (Lemmas 5.2 and 5.3). It will be explained in Remark 5.4 why the second subcase of (ii) does not contradict the general theory developed in [11]. In this paper we do not pay further attention to the $\gamma$-eigenvalues near $\lambda_{2 k+1}^{r}<0, k \geq 1$ (that only exist for $1<\beta_{2}<2$ $(3.7))$.

Proof. In the proof we consider the balls $B\left(\lambda_{j}^{r}, \tilde{\delta}\right)$ around $\lambda_{j}^{r}$ with radius $\tilde{\delta}>\delta$ with $\delta$ as defined in (1.8), so that $\partial B\left(\lambda_{j}^{r}, \tilde{\delta}\right) \subset \mathbb{C}_{r}$. With a slight abuse of notation we do not distinguish 
between this $\tilde{\delta}$ and $\delta$, i.e. we assume that $\partial B\left(\lambda_{j}^{r}, \delta\right) \subset \mathbb{C}_{r}$ for the $\delta$ that appears in the Lemma.

(i) The (leading order) condition (1.10) on the existence of $\gamma$-eigenvalues can be written as

$$
\frac{\sqrt{\mu}}{\sqrt{\mu+\lambda}}\left[\alpha_{1}-\alpha_{2} \beta_{1} \mathcal{R}\left(\lambda ; \beta_{1}, \beta_{2}\right)\right]=-\frac{1-2 \Delta^{2} \operatorname{Re}[\gamma]+\Delta^{4}}{\left(1-\Delta^{4}\right) \tanh \left(\varepsilon^{2} \sqrt{\mu} L\right)} .
$$

If $\lambda$ is $\mathcal{O}(\delta)$ close to an even eigenvalue $\lambda_{2 k}^{r}$, the absolute value of the left hand side of this identity clearly is $\gg 1$ with respect to $\delta$, since $\mathcal{R}(\lambda)$ has a simple pole at $\lambda_{2 k}^{r}$, i.e. $|\mathcal{R}(\lambda)|=\mathcal{O}(1 / \delta)$ and $\mu \gg \delta,\left|\alpha_{1}\right|=\mathcal{O}(1), \alpha_{2} \gg \sqrt{\delta} ; \varepsilon^{2} L=\mathcal{O}(1), 0<|\Delta|<1$ and $|1-\Delta|=\mathcal{O}(1)$ by the assumption on $L$, so that the right hand side is $\mathcal{O}(1)$ with respect to $\delta$ for $\gamma \in \mathbf{S}^{1}$. This implies by Proposition 3.8 that $\mathcal{D}(\lambda, \gamma)$ cannot have zeroes on $\partial B\left(\lambda_{j}^{r}, \delta\right)$. Hence, for any (fixed) $\gamma \in \mathbf{S}^{1}$, we can define the winding number $\mathcal{W}\left(\mathcal{D}, \partial B\left(\lambda_{j}^{r}, \delta\right)\right)$ of $\mathcal{D}(\lambda, \gamma)$ over the contour $\partial B\left(\lambda_{j}^{r}, \delta\right)$. Since $\mathcal{D}(\lambda, \gamma)$ is analytic as function of $\lambda$ in $\left.B\left(\lambda_{j}^{r}, \delta\right)\right), \mathcal{W}\left(\mathcal{D}, \partial B\left(\lambda_{j}^{r}, \delta\right)\right)=$ the number of zeroes of $\mathcal{D}(\lambda, \gamma)=$ the number of $\gamma$-eigenvalues of $(2.2)$ for this given $\gamma$ inside $\left.B\left(\lambda_{j}^{r}, \delta\right)\right)$.

On $\partial B\left(\lambda_{j}^{r}, \delta\right), \mathcal{D}^{f}(\lambda, \gamma)$ and $\mathcal{D}^{s}(\lambda, \gamma)$ are defined (and analytic), $\mathcal{D}(\lambda, \gamma)$ can be decomposed into the product $\mathcal{D}^{f}(\lambda, \gamma) \mathcal{D}^{s}(\lambda, \gamma)$, and the approximations given in (3.19) are uniform in $\lambda, \gamma, L$ - Proposition 3.8 (in combination with the uniform approximations of $t_{i j}(\lambda)$ in Section 4.2). Thus,

$$
\begin{aligned}
\mathcal{W}(\mathcal{D}, \partial B) & =\frac{1}{2 \pi i} \oint_{\partial B} \frac{\frac{d}{d \lambda} \mathcal{D}(\lambda, \gamma)}{\mathcal{D}(\lambda, \gamma)} d \lambda \\
& =\frac{1}{2 \pi i} \oint_{\partial B} \frac{\frac{d}{d \lambda} \mathcal{D}^{f}(\lambda, \gamma)}{\mathcal{D}^{f}(\lambda, \gamma)} d \lambda+\frac{1}{2 \pi i} \oint_{\partial B} \frac{\frac{d}{d \lambda} \mathcal{D}^{s}(\lambda, \gamma)}{\mathcal{D}^{s}(\lambda, \gamma)} d \lambda \\
& =\left[\oint_{\partial B} \frac{\frac{d}{d \lambda}\left(-\gamma t^{r}(\lambda) e^{2 \Lambda^{f} L}\right)}{-\gamma t^{r}(\lambda) e^{2 \Lambda^{f} L}} d \lambda+\oint_{\partial B} \frac{\frac{d}{d \lambda}\left(\gamma^{2}-\left(t_{11}(\lambda, 0) / \Delta^{2}+\Delta^{2} t_{22}(\lambda, 0)\right) \gamma+1\right)}{\gamma^{2}-\left(t_{11}(\lambda, 0) / \Delta^{2}+\Delta^{2} t_{22}(\lambda, 0)\right) \gamma+1} d \lambda\right] \frac{1+\mathcal{O}(\varepsilon)}{2 \pi i} \\
& =\mathcal{W}\left(t^{r}(\lambda), \partial B\right)+\mathcal{W}\left(\gamma^{2}-\gamma \operatorname{Tr}\left(M^{s}(\lambda, 0)\right)+1, \partial B\right)+\mathcal{O}(\varepsilon)
\end{aligned}
$$

(3.19), (3.20),(1.10). The transmission function $t^{r}(\lambda)$ is analytic in $\left.B\left(\lambda_{2 k}^{r}, \delta\right)\right)$ and has a unique zero at $\lambda_{2 k}^{r}\left([3]\right.$, Section 3.1), so that $\mathcal{W}\left(t^{r}(\lambda), \partial B\right)=1$. The trace $\operatorname{Tr}\left(M^{s}(\lambda, 0)\right)$ depends on $\lambda$ through $\mathcal{R}(\lambda)(1.10)$ and is thus meromorphic on $\left.B\left(\lambda_{2 k}^{r}, \delta\right)\right)$ with a simple pole at $\lambda_{2 k}^{r}([3]$, Section 4.1). By the conditions on the parameters $\mu$ and $\alpha_{1,2}$, it follows that $\left|\operatorname{Tr}\left(M^{s}(\lambda, 0)\right)\right| \gg 1$ with respect to $\delta$ on $\left.B\left(\lambda_{2 k}^{r}, \delta\right)\right)$, so that for $\gamma \in \mathbf{S}^{1}$,

$$
\mathcal{W}\left(\gamma^{2}-\gamma \operatorname{Tr}\left(M^{s}(\lambda, 0)\right)+1, \partial B\right)=\mathcal{W}\left(\operatorname{Tr}\left(M^{s}(\lambda, 0)\right), \partial B\right)=\mathcal{W}(\mathcal{R}(\lambda), \partial B)=-1 .
$$

Hence, for any $\gamma \in \mathbf{S}^{1}$,

$$
\mathcal{W}(\mathcal{D}, \partial B)=\mathcal{W}\left(t^{r}(\lambda), \partial B\right)+\mathcal{W}(\mathcal{R}(\lambda), \partial B)+\mathcal{O}(\varepsilon)=1-1+\mathcal{O}(\varepsilon)=0,
$$

since $\mathcal{W}(\mathcal{D}, \partial B) \in \mathbb{Z}$ (and $0<\varepsilon \ll 1)$. This proves that there are no $\gamma$-eigenvalues in $B\left(\lambda_{2 k}^{r}, \delta\right)$.

(ii) Both the slow Evans function $\mathcal{D}^{s}(\lambda, \gamma)$ and $\operatorname{Tr}\left(M^{s}(\lambda, 0)\right)$ are smooth as function of $\lambda$ on $\partial B\left(\lambda_{2 k+1}^{r}, \delta\right)(1.10)$, so that

$$
\mathcal{D}^{s}(\lambda, \gamma)=\gamma^{2}-\gamma \operatorname{Tr}\left(M^{s}(\lambda, 0)\right)+1+\mathcal{O}(\varepsilon) \equiv \gamma^{2}-\gamma \operatorname{Tr}\left(M^{s}\left(\lambda_{2 k+1}^{r}, 0\right)\right)+1+\mathcal{O}(\delta)
$$

for $\lambda \in \partial B\left(\lambda_{2 k+1}^{r}, \delta\right)$ (Proposition 3.8). The condition $\operatorname{Tr}\left(M^{s}\left(\lambda_{2 k+1}^{r}, 0\right)\right) \notin[-2,2]$ implies that $\gamma^{2}-\gamma \operatorname{Tr}\left(M^{s}(\lambda, 0)\right)+1 \neq 0$ for all $\gamma \in \mathbf{S}^{1}$. If the condition holds, this is true for all $\lambda \in B\left(\lambda_{2 k+1}^{r}, \delta\right)$ since $\operatorname{Tr}\left(M^{s}(\lambda, 0)\right)$ is well-defined and smooth on $B\left(\lambda_{2 k+1}^{r}, \delta\right)$, unlike $\mathcal{D}^{s}(\lambda, \gamma)$. The approach of the proof of $(i)$ can now be applied without any modification, up to the arguments leading to $(4.21)$, since now clearly $\mathcal{W}\left(\gamma^{2}-\gamma \operatorname{Tr}\left(M^{s}(\lambda, 0)\right)+1, \partial B\right)=0$. This yields,

$$
\mathcal{W}(\mathcal{D}, \partial B)=\mathcal{W}\left(t^{r}(\lambda), \partial B\right)+\mathcal{W}\left(\gamma^{2}-\gamma \operatorname{Tr}\left(M^{s}(\lambda, 0)\right)+1, \partial B\right)+\mathcal{O}(\varepsilon)=1+\mathcal{O}(\varepsilon)=1,
$$

so that there indeed is a unique $\gamma$-eigenvalue $\in B\left(\lambda_{2 k+1}^{r}, \delta\right)$ for any $\gamma \in \mathbf{S}^{1}$ if $\operatorname{Tr}\left(M^{s}\left(\lambda_{2 k+1}^{r}, 0\right)\right) \notin$ $[-2,2]$. Since complex eigenvalues of $(2.2)$ come in pairs, it follows that $\lambda(\gamma) \in \mathbb{R}$.

If $\operatorname{Tr}\left(M^{s}\left(\lambda_{2 k+1}^{r}, 0\right)\right)=\operatorname{Tr}^{*} \in[-2,2]$, then $\gamma^{2}-\gamma \operatorname{Tr}\left(M^{s}(\lambda, 0)\right)+1=0$ has two solutions $\gamma_{1,2}^{*} \in \mathbf{S}^{1}$ with $\operatorname{Re}\left[\gamma_{1,2}^{*}\right]=\frac{1}{2} \operatorname{Tr}^{*}$. Hence, for any $\gamma \in \mathbf{S}^{1}$ with $\left|\gamma-\gamma_{1,2}^{*}\right|=\mathcal{O}(\delta)$, 
$\gamma^{2}-\gamma \operatorname{Tr}\left(M^{s}(\lambda, 0)\right)+1=\mathcal{O}(\delta)$ as function of $\lambda \in B\left(\lambda_{2 k+1}^{r}, \delta\right)$. As a consequence, we do not know the number of zeroes of $\gamma^{2}-\gamma \operatorname{Tr}\left(M^{s}(\lambda, 0)\right)+1$ in $B\left(\lambda_{2 k+1}^{r}, \delta\right)$ for these $\gamma$ 's. Nevertheless, if $\left|\gamma-\gamma_{1,2}^{*}\right| \gg \delta$ then clearly $\gamma^{2}-\gamma \operatorname{Tr}\left(M^{s}(\lambda, 0)\right)+1 \neq 0$ for $\lambda \in B\left(\lambda_{2 k+1}^{r}, \delta\right)$ and (4.22) can be applied.

Remark 4.4 If $\varepsilon^{2} L$ becomes small, the right hand side of (4.20) is large (if $\gamma$ is not too close to 1$)$, so that $(1.10)$ will have solutions for $\mathcal{R}(\lambda)$ large, i.e that there are $\gamma$-eigenvalues $(\gamma \neq 1)$ near the poles of $\mathcal{R}(\lambda)$. Hence, the periodic patterns of type B must be unstable for $\varepsilon^{2} L_{B}$ small enough, since these patterns have $\gamma$-eigenvalues $(\gamma \neq 1)$ close to $\lambda_{0}^{r}>0$. Here, we will not work out the details of this statement. In Corollary 6.6 it will be shown that all patterns of type B are unstable.

Remark 4.5 The conditions in Proposition 4.3 on the parameters $\mu$ and $\alpha_{1,2}$ can slightly be weakened. Nevertheless, as in [3] they correspond directly to the instability results. It is shown in Theorems 5.1 and 5.2 of [3] that the homoclinic pulse is unstable if $\mu,\left|\alpha_{2}\right|$ is too small, or $\left|\alpha_{1}\right|$ too large. Equivalently, there will be spectrum near the even eigenvalues $\lambda_{j}^{r}$ under these circumstances, i.e. the long wave periodic pattern will be unstable if $\mu<\mu_{c},\left|\alpha_{1}\right|>\alpha_{1, c}$ or $\left|\alpha_{2}\right|<\alpha_{2, c}$ for some $\mu_{c}, \alpha_{1, c}, \alpha_{2, c}>0$.

\section{The small eigenvalues}

Proposition 4.3 established the existence of $\gamma$-eigenvalues near $0=\lambda_{1}^{r}$. Since the derivative of the wave is a 1-eigenfunction for $\lambda=0$, there exists a curve of small $\gamma$-eigenvalues connected to $\lambda=0$ (Proposition 2.2).

\subsection{An explicit expression for $\lambda=\lambda(\gamma)$}

Let $\phi_{\gamma}$ be a $\gamma$-eigenfunction, so that $\phi_{\gamma}$ satisfies $\phi_{\gamma}(L)=\gamma \phi_{\gamma}(-L)$. Asymptotically close to $\lambda=0$, the $\gamma$-eigenfunctions is expected to be similar to the derivative of the wave, however, since $\dot{U}_{p}(\xi)$ is $2 L$-periodic, it can not satisfy the $\gamma$-boundary conditions if $\gamma \neq 1$. Therefore, we have to add a perturbation to the $u$ component of $\phi_{\gamma}$ that is of the same order $\left(\mathcal{O}\left(\varepsilon^{2}\right)\right)$ as $\dot{U}_{p}$. Since the leading order perturbation in the fast $v$-component follows from the order of the perturbation in $u$ (through $(2.1)$ ), we also add an $\mathcal{O}\left(\varepsilon^{2}\right)$ perturbation to $\dot{V}_{p}$. Therefore, we propose in our search for the small eigenvalues the following expansions for the $u$ and $v$ components of $\phi_{\gamma}$

$$
u_{\gamma}(\xi)=\dot{U}_{p}(\xi)+\varepsilon^{2} u_{1}(\xi ; \varepsilon) \text { and } v_{\gamma}(\xi)=\dot{V}_{p}(\xi)+\varepsilon^{2} v_{1}(\xi ; \varepsilon),
$$

with the $\gamma$-boundary conditions

$$
u_{\gamma}(L)=\gamma u_{\gamma}(-L) \quad \text { and } \quad \dot{u}_{\gamma}(L)=\gamma \dot{u}_{\gamma}(-L) .
$$

Although it is quite natural to expect that the $\gamma$-eigenfunctions have this structure for $\lambda$ close to 0 , it is a priori not necessary. However, if we find small $\gamma$-eigenvalues by 'ansatz' (5.1), then we know by Proposition 4.3 that all $\gamma$-eigenfunctions must be of the form (5.1).

It is again not necessary to explicitly consider the $\gamma$-boundary conditions on the fast components of $\phi_{\gamma}$. In fact, the choice of $\dot{V}_{p}(\xi)$ as the leading order part of $v_{\gamma}$ is not a priori obvious, since $\dot{V}_{p}(\xi)$ does not satisfy the $\gamma$-boundary conditions. However, it has been argued in Section 4.2 that $v_{\gamma}$ can be approximated (uniformly in $\mathbb{R}$ ) by a summation of shifted localized solutions (4.19), i.e. the leading order approximation of $v_{\gamma}$ is exponentially small outside the pulse region(s). Here, the leading order part of $v_{i n}(\xi)$ in $(4.19)$ is given by $\dot{V}_{p}(\xi)$, since $u_{0}^{s}=$ the leading 
order part of $\dot{U}_{p}(\xi)=\mathcal{O}\left(\varepsilon^{2}\right)$ in (4.5), so that there is no inhomogeneous term in the leading order $v_{i n}$-equation (4.6). Note that the higher order terms of $v_{\gamma}$ can also be written in the form (4.19), $v_{1}$ is exponentially small in all outer regions. On the other hand it is not useful to write the slow part of $\phi_{\gamma}$ in a form similar to (4.19). Since the $u$-component of an eigenfunction to (2.1) decays only on an $\mathcal{O}\left(\varepsilon^{-2}\right)$-length scale, the terms coming from $n \neq 0$ give a leading order contribution to the $u$-component on $[-L, L]$. Instead we consider the $u$-component directly in the first equation of (2.1), with its explicit $\gamma$-boundary conditions.

For notational convenience, we introduce the operator $\mathcal{L}_{\varepsilon}^{f}$ for the left hand side in the second equation of $(2.1)$

$$
\mathcal{L}_{\varepsilon}^{f}=\frac{d^{2}}{d \xi^{2}}+\left[\beta_{2} U_{p}^{\alpha_{2}} V_{p}^{\beta_{2}-1}-1\right]
$$

so that $v_{\gamma}$ must solve

$$
\left(\mathcal{L}_{\varepsilon}^{f}-\lambda\right) v_{\gamma}=-\alpha_{2} U_{p}^{\alpha_{2}-1} V_{p}^{\beta_{2}} u_{\gamma} .
$$

Since the pair $\left(\dot{U}_{p}(\xi), \dot{V}_{p}(\xi)\right)$ solves the eigenvalue problem $(2.1)$ for $\lambda=0$, the operator $\mathcal{L}_{\varepsilon}^{f}$ satisfies the useful relationship

$$
\mathcal{L}_{\varepsilon}^{f} \dot{V}_{p}(\xi)=-\alpha_{2} U_{p}^{\alpha_{2}-1} V_{p}^{\beta_{2}} \dot{U}_{p}(\xi)
$$

Note that this is an exact expression, i.e. we have not introduced any approximations of $U_{p}(\xi)$ and of $V_{p}(\xi)$. We substitute the expansions of $u_{\gamma}$ and $v_{\gamma}(5.1)$ in the eigenvalue problem (2.1). After elimination of the terms containing $\dot{U}_{p}$ and $\dot{V}_{p}$ in the resulting expression, the linear problem for $u_{1}(\xi)$ and $v_{1}(\xi)$ reads

$$
\left\{\begin{aligned}
\ddot{u}_{1} & =-\varepsilon^{2}\left[\alpha_{1} U_{p}^{\alpha_{1}-1} V_{p}^{\beta_{1}} u_{1}+\beta_{1} U_{p}^{\alpha_{1}} V_{p}^{\beta_{1}-1} v_{1}\right]+\varepsilon^{4}(\mu+\lambda) u_{1} \\
\mathcal{L}_{\varepsilon}^{f} v_{1} & =\frac{\lambda}{\varepsilon^{2}} \dot{V}_{p}-\alpha_{2} U_{p}^{\alpha_{2}-1} V_{p}^{\beta_{2}} u_{1}
\end{aligned}\right.
$$

Furthermore, the $\gamma$-boundary conditions on $u_{\gamma}$ yield

$$
\begin{aligned}
\dot{U}_{p}(L)+\varepsilon^{2} u_{1}(L) & =\gamma\left(\dot{U}_{p}(-L)+\varepsilon^{2} u_{1}(-L)\right) \\
\ddot{U}_{p}(L)+\varepsilon^{2} \dot{u}_{1}(L) & =\gamma\left(\ddot{U}_{p}(-L)+\varepsilon^{2} \dot{u}_{1}(-L)\right) .
\end{aligned}
$$

So far, we have not considered the magnitude (with respect to $\varepsilon$ ) of $\lambda$. The second equation of (5.2) suggests that $\lambda=\mathcal{O}\left(\varepsilon^{2}\right)$. However, we will derive a solvability condition for the second equation of (5.2) to show that $\lambda=\mathcal{O}\left(\varepsilon^{4}\right)$ and to derive an explicit expression for $\lambda(\gamma)$. Therefore, we rewrite the second equation of (5.2) as a problem involving the operator $\mathcal{L}^{r}(3.4)$. We approximate the pulse around $\xi=0$ by

$$
V_{p}(\xi)=U_{0}^{-\alpha_{2} /\left(\beta_{2}-1\right)} w_{h}(\xi)
$$

where $U_{0}$ is the leading order (constant) value of $U_{p}(\xi)$ in the pulse region (Theorem 1.7), and $w_{h}(\xi)$ is defined in (1.20). Thus, $\mathcal{L}_{\varepsilon}^{f}=\mathcal{L}^{r}+$ higher order terms, and the leading order part of the second equation of (5.2) is given by the inhomogeneous equation

$$
\mathcal{L}^{r} w_{1}=\frac{\lambda}{\varepsilon^{2}} U_{0}^{-\alpha_{2} /\left(\beta_{2}-1\right)} \dot{w}_{h}-\alpha_{2} U_{0}^{-1-\alpha_{2} /\left(\beta_{2}-1\right)} w_{h}^{\beta_{2}} u_{1},
$$

where $w_{1}$ denotes the leading order part of $v_{1}$. The homogeneous problem $\mathcal{L}^{r} w=0$ is solved by $\dot{w}_{h}$ (Section 3.1), so that we have the following leading order solvability condition for (5.3)

$$
\left\langle\dot{w}_{h} \mid \frac{\lambda}{\varepsilon^{2}} U_{0}^{-\alpha_{2} /\left(\beta_{2}-1\right)} \dot{w}_{h}-\alpha_{2} U_{0}^{-1-\alpha_{2} /\left(\beta_{2}-1\right)} w_{h}^{\beta_{2}} u_{1}\right\rangle=0
$$

where $\langle f \mid g\rangle$ is the standard $L_{2}$-inner product on $\mathbb{R}$. Since $U_{0}$ is constant we find

$$
\frac{\lambda}{\varepsilon^{2}} U_{0}^{-\alpha_{2} /\left(\beta_{2}-1\right)}\left\langle\dot{w}_{h} \mid \dot{w}_{h}\right\rangle-\alpha_{2} U_{0}^{-1-\alpha_{2} /\left(\beta_{2}-1\right)}\left\langle\dot{w}_{h} \mid w_{h}^{\beta_{2}} u_{1}\right\rangle=0
$$


which yields (at leading order),

$$
\lambda=\varepsilon^{2} \alpha_{2} \frac{1}{U_{0}} \frac{\left\langle\dot{w}_{h} \mid w_{h}^{\beta_{2}} u_{1}\right\rangle}{\left\langle\dot{w}_{h} \mid \dot{w}_{h}\right\rangle}
$$

It follows from (5.2), that $\ddot{u}_{1}=\mathcal{O}\left(\varepsilon^{4}\right)$ in the outer regions and $\ddot{u}_{1}=\mathcal{O}\left(\varepsilon^{2}\right)$ in the pulse region. Therefore, the first derivative $\dot{u}_{1}$ is at most $\mathcal{O}\left(\varepsilon^{2}\right)$, so that $u_{1}$ is to leading order even. However, since $\dot{w}_{h}$ is an odd function, this even leading order term gives no contribution to the integral in the numerator, so that we need to take the (odd) higher order terms into account. To approximate the integral in the numerator, we substitute the leading order Taylor expansion of $u_{1}(\xi)$ around $\xi=0$,

$$
\lambda=\alpha_{2} \frac{1}{U_{0}} \frac{\left\langle\dot{w}_{h}\right| w_{h}^{\beta_{2}}\left(u_{1}(0)+\dot{u}_{1}(0) \xi\right\rangle}{\left\langle\dot{w}_{h} \mid \dot{w}_{h}\right\rangle}=\alpha_{2} \frac{1}{U_{0}} \frac{\left\langle\dot{w}_{h} \mid w_{h}^{\beta_{2}} \xi\right\rangle}{\left\langle\dot{w}_{h} \mid \dot{w}_{h}\right\rangle} \dot{u}_{1}(0),
$$

Note that the third and higher derivatives of $u_{1}$ are $\mathcal{O}\left(\varepsilon^{4}\right)$ or smaller, as can be verified by taking the derivative of the first equation of (5.2) and evaluation of the resulting expression in $\xi=0$. Finally, using integration by parts, the expression for $\lambda$ is written as

$$
\lambda(\gamma)=\varepsilon^{2} \frac{\left|\alpha_{2}\right|}{\beta_{2}+1} \frac{1}{U_{0}} \frac{\int w_{h}^{\beta_{2}+1} d \xi}{\int \dot{w}_{h}^{2} d \xi} \dot{u}_{1}(0 ; \gamma),
$$

where the dependence on $\gamma$ is only due to $\dot{u}_{1}(0 ; \gamma)$. From this expression for $\lambda$ we conclude two things. First, since $\dot{u}_{1}(0 ; \gamma)=\mathcal{O}\left(\varepsilon^{2}\right)$, we see that $\lambda=\mathcal{O}\left(\varepsilon^{4}\right)$ and second that $\lambda$ is proportional to the derivative $\dot{u}_{1}(0)$. A careful error-analysis of the above leading order arguments yields that the leading order correction to $(5.4)$ is $\mathcal{O}\left(\varepsilon^{5}\right)$.

Remark 5.1 The expression for $\lambda$ was derived as a solvability condition for the leading order part of $v_{1}$. Alternatively the same result can be found directly from the full equation for $v_{1}$ in (5.2) by multiplying both sides of the second equation of (5.2) by $\dot{V}_{p}(\xi)$ and integrating the result over one period

$$
\int_{-L}^{+L} \dot{V}_{p} \mathcal{L}_{\varepsilon}^{f} v_{1} d \xi=\frac{\lambda}{\varepsilon^{2}} \int_{-L}^{+L} \dot{V}_{p} \dot{V}_{p} d \xi-\alpha_{2} \int_{-L}^{+L} \dot{V}_{p} U_{p}^{\alpha_{2}-1} V_{p}^{\beta_{2}} u_{1} d \xi .
$$

The crucial point in this calculation is to show that the integral on the left hand side is $\mathcal{O}\left(\varepsilon^{4}\right)$, whereas the second integral on the right hand side is $\mathcal{O}\left(\varepsilon^{2}\right)$. The latter is easily shown by substituting the Taylor series expansion of $u_{1}$ in the integral, as was done above. The former requires somewhat more work.

\subsection{The position of the $\mathcal{O}\left(\varepsilon^{4}\right)$ spectrum}

Since we know that $\lambda(\gamma)=\mathcal{O}\left(\varepsilon^{4}\right)$, we can at leading order neglect the terms containing $\lambda$ from (5.2), so that the linear problem for the leading order parts of $u_{1}$ and $v_{1}$ is written as

$$
\left\{\begin{aligned}
\ddot{u}_{1} & =-\varepsilon^{2}\left[\alpha_{1} U_{p}^{\alpha_{1}-1} V_{p}^{\beta_{1}} u_{1}+\beta_{1} U_{p}^{\alpha_{1}} V_{p}^{\beta_{1}-1} v_{1}\right]+\varepsilon^{4} \mu u_{1} \\
\mathcal{L}_{\varepsilon}^{f} v_{1} & =-\alpha_{2} U_{p}^{\alpha_{2}-1} V_{p}^{\beta_{2}} u_{1}
\end{aligned}\right.
$$

Thus, the leading order parts of $u_{1}$ and $v_{1}$ satisfy the linear eigenvalue problem (2.1) for $\lambda=0$. The set of solutions for $u_{1}$ and $v_{1}$ to (5.5) is at leading order the same as the set of solutions of (2.1) for $\lambda=0$. We showed in Section 3 that eigenvalue problem (2.1) has two types of solutions that have $v$-components that are exponentially small in the outer regions $\mathcal{I}_{ \pm}$. The derivative of the wave $\left(\dot{U}_{p}, \dot{V}_{p}\right)$ is a fast solution of (2.1) for $\lambda=0$ - Section 3.1. Since this solution can be absorbed in the leading order part of $u$ and $v$ (5.1), it does not contribute to $\left(u_{1}, v_{1}\right)$. Therefore, the solution of (5.5) must be $\in \Phi^{s}(\xi, \lambda)$ (Lemma 3.6), i.e. $\left(u_{1}(\xi ; \varepsilon), v_{1}(\xi ; \varepsilon)\right)$ is at leading order 
spanned by the slow solutions $\left\{\left(u_{1}^{s}, v_{1}^{s}\right),\left(u_{2}^{s}, v_{2}^{s}\right)\right\}$, so that there exist constants $c_{1}$ and $c_{2}$ such that

$$
u_{1}(\xi ; \varepsilon)=c_{1} u_{1}^{s}(\xi)+c_{2} u_{2}^{s}(\xi)+\mathcal{O}(\varepsilon)
$$

for all $\xi \in[-L, L]$. The constants $c_{1}$ and $c_{2}$ are determined by the $\gamma$-boundary conditions. We now need to express $\dot{u}_{1}(0 ; \gamma)$ in terms of $c_{1}$ and $c_{2}$. We integrate $\ddot{u}_{1}(\xi)$ over the left half of $\mathcal{P}_{0}$ and obtain

$$
\dot{u}_{1}(0)=\dot{u}_{1}\left(-\frac{1}{\sqrt{\varepsilon}}\right)+\int_{-\frac{1}{\sqrt{\varepsilon}}}^{0} \ddot{u}_{1} d \xi .
$$

Since the leading order part of $\ddot{u}_{1}$ is an even function (see (5.2) and recall that the leading order parts of $u_{1}$ and $v_{1}$ are even), we have

$$
\int_{-\frac{1}{\sqrt{\varepsilon}}}^{0} \ddot{u}_{1} d \xi=\frac{1}{2} \int_{-\frac{1}{\sqrt{\varepsilon}}}^{\frac{1}{\sqrt{\varepsilon}}} \ddot{u}_{1} d \xi+\text { h.o.t. }=\frac{1}{2} \mathcal{J}(0) u_{1}(0)+\text { h.o.t. }
$$

(4.3), (4.4). By (3.12) and Lemma 3.6 we thus have

$$
\dot{u}_{1}(0)=\varepsilon^{2} \sqrt{\mu}\left(c_{1}-c_{2}\right)+\frac{1}{2}\left(c_{1}+c_{2}\right) \mathcal{J}(0)+\text { h.o.t. }=\mathcal{O}\left(\varepsilon^{2}\right)
$$

(4.15). For notational convenience we re-introduce the vector notation of the previous sections, i.e. the $\gamma$-eigenfunction $\phi_{\gamma}=\left(u_{\gamma}, \dot{u}_{\gamma}, v_{\gamma}, \dot{v}_{\gamma}\right)^{t}$ is expanded as

$$
\phi_{\gamma}(\xi)=\phi_{0}(\xi)+\varepsilon^{2} \phi_{1}(\xi ; \varepsilon)
$$

where $\phi_{0}=\left(\dot{U}_{p}, \ddot{U}_{p}, \dot{V}_{p}, \ddot{V}_{p}\right)^{t}$ and $\phi_{1}=\left(u_{1}, \dot{u}_{1}, v_{1}, \dot{v}_{1}\right)^{t}$ is at leading order given by $\phi_{1}(\xi)=$ $c_{1}(\gamma) \phi_{1}^{s}(\xi)+c_{2}(\gamma) \phi_{2}^{s}(\xi)$, with $c_{1,2}$ as above. As in Section 3.3, we decompose $\phi_{\gamma}(-L)$ and $\phi_{\gamma}(+L)$ with respect to $V=\left\{\mathbf{v}_{1}^{s}, \mathbf{v}_{2}^{s}\right\}=\left\{\phi_{1}^{s}(-L), \phi_{2}^{s}(-L)\right\}$ (3.17), with, by (3.12),

$$
\mathbf{v}_{1}^{s}=e^{-\Lambda^{s}(0) L} E_{+}^{s}(0)=e^{-\varepsilon^{2} \sqrt{\mu} L}\left(\begin{array}{c}
1 \\
\varepsilon^{2} \sqrt{\mu} \\
0 \\
0
\end{array}\right) \text { and } \mathbf{v}_{2}^{s}=e^{+\varepsilon^{2} \sqrt{\mu} L}\left(\begin{array}{c}
1 \\
-\varepsilon^{2} \sqrt{\mu} \\
0 \\
0
\end{array}\right)
$$

up to exponentially small corrections. By $(3.11), \phi_{1}(L)$ is given by

$$
\phi_{1}(L)=c_{1} \phi_{1}^{s}(L)+c_{2} \phi_{2}^{s}(L)=\left(c_{1} m_{11}+c_{2} m_{12}\right) v_{1}^{s}+\left(c_{1} m_{21}+c_{2} m_{22}\right) v_{2}^{s},
$$

with $m_{i j}=m_{i j}(0)$ as in (4.18), and, up to exponentially small corrections, $\phi_{0}( \pm L)$ by

$$
\phi_{0}( \pm L)=\left(\begin{array}{c}
\dot{U}_{p}(-L) \\
\ddot{U}_{p}(-L) \\
0 \\
0
\end{array}\right)=\left(\begin{array}{c}
0 \\
\varepsilon^{4} \mu U_{\min } \\
0 \\
0
\end{array}\right)=\varepsilon^{2} \sqrt{\mu} U_{\min }\left(\frac{1}{\Delta_{0}} \mathbf{v}_{1}^{s}-\Delta_{0} \mathbf{v}_{2}^{s}\right)
$$

where $U_{\min }=U_{\min }(\nu)$ is the minimal value of $U_{p}(\xi)(1.17)$ and $\Delta_{0}=\Delta(0, L)(1.11)$. Combining (5.8), (5.9) and (5.10) yields,

$$
\begin{gathered}
\phi_{\gamma}(-L)=\varepsilon^{2}\left(\sqrt{\mu} U_{\min } \frac{1}{\Delta_{0}}+c_{1}\right) \mathbf{v}_{1}^{s}(-L)+\varepsilon^{2}\left(\sqrt{\mu} U_{\min } \Delta_{0}+c_{2}\right) \mathbf{v}_{2}^{s}(-L) \\
\phi_{\gamma}(L)=\varepsilon^{2}\left(\sqrt{\mu} U_{\min } \frac{1}{\Delta_{0}}+d_{1}\right) \mathbf{v}_{1}^{s}(-L)+\varepsilon^{2}\left(\sqrt{\mu} U_{\min } \Delta_{0}+d_{2}\right) \mathbf{v}_{2}^{s}(-L),
\end{gathered}
$$

where $d_{1}$ and $d_{2}$ are related to $c_{1}$ and $c_{2}$ by

$$
\left(\begin{array}{l}
d_{1} \\
d_{2}
\end{array}\right)=M^{s}(0,0)\left(\begin{array}{l}
c_{1} \\
c_{2}
\end{array}\right)
$$


(4.18). The $\gamma$-boundary condition $\phi_{\gamma}(L)=\gamma \phi_{\gamma}(-L)$ induces the following leading order equations for $c_{1,2}$,

$$
\begin{aligned}
\sqrt{\mu} U_{\min } \frac{1}{\Delta_{0}}+d_{1} & =\gamma\left(\sqrt{\mu} U_{\min } \frac{1}{\Delta_{0}}+c_{1}\right) \\
-\sqrt{\mu} U_{\min } \Delta_{0}+d_{2} & =\gamma\left(-\sqrt{\mu} U_{\min } \Delta_{0}+c_{2}\right)
\end{aligned},
$$

which is equivalent to an inhomogeneous matrix problem,

$$
\left(M^{s}(0,0)-\gamma I\right)\left(\begin{array}{l}
c_{1} \\
c_{2}
\end{array}\right)=(\gamma-1) \sqrt{\mu} U_{\min }\left(\begin{array}{c}
\frac{1}{\Delta_{0}} \\
-\Delta_{0}
\end{array}\right)
$$

(5.11). We conclude that we have gained full control over the $\mathcal{O}\left(\varepsilon^{4}\right) \gamma$-eigenvalues: they are at leading order given by (5.4), with $\dot{u}_{1}(0 ; \gamma)$ as in (5.7), and $c_{1}$ and $c_{2}$ determined by (5.12) and (4.18). Moreover, we know by the reversibility arguments of Section 2 that $\lambda(\gamma)=\lambda(\bar{\gamma})$ and that $u_{1}(\xi ; \gamma)=u_{1}(\xi ; \bar{\gamma})$ - recall that $\xi=0$ has been set as a point of symmetry. Thus, $c_{1,2}(\bar{\gamma})=$ $c_{1,2}(\gamma)$ by (5.6). Since $\gamma \in \mathbf{S}^{1}$ is the only non-real ingredient in the $\gamma$-boundary conditions that determine $c_{1,2}$, we also know that $c_{1,2}(\bar{\gamma})=\overline{c_{1,2}(\gamma)}$ (exactly). Hence, $c_{1,2}(\gamma)=\overline{c_{1,2}(\gamma)}$ and we may conclude that the small eigenvalues are real, i.e. $\lambda(\gamma) \in \mathbb{R}$.

Lemma 5.2 Suppose that the eigenvalues $\rho$ and $1 / \rho$ of $M^{s}(0,0)$ are not in $\mathbf{S}^{1}$. Then, the small $\gamma$-eigenvalues have the following properties (for all $\left.\gamma \in \mathbf{S}^{1}\right): \lambda(\gamma) \in \mathbb{R}, \lambda(\gamma)=\mathcal{O}\left(\varepsilon^{4}\right)$, $\lambda(-1)<0, \lambda(1)=0$ has multiplicity 1 , and $\lambda(-1) \leq \lambda(\gamma) \leq \lambda(1)=0$.

Proof. We know by Proposition 4.3 that there must be $\gamma$-eigenvalues and $\gamma$-eigenfunctions within the ball $B(0, \delta)(1.9)$. The above analysis is based on our control of the full set of independent solutions of the linear eigenvalue problem (2.2) (for $\lambda=0$ ), as developed in Sections 3 and 4 . We have constructed the small $\gamma$-eigenfunctions and we know that $\lambda(\gamma) \in \mathbb{R}$ and $\lambda(\gamma)=\mathcal{O}\left(\varepsilon^{4}\right)$. For $\gamma=-1, c_{1}$ and $c_{2}$ do at leading order not depend on $\mathcal{J}(0)$,

$$
c_{1}=-c_{2}=-2 \sqrt{\mu} U_{\min } \frac{\Delta_{0}}{1+\Delta_{0}^{2}}
$$

(5.12). Since $c_{1}+c_{2}=0$, the slow derivative $\dot{u}_{1}(0)(5.7)$ is also independent of $\mathcal{J}(0)$, so that the sign of $\dot{u}_{1}(0)$, and hence the position of the small $\gamma$-eigenvalue $(5.4)$, is determined by the sign of $c_{1}-c_{2}$. We know by (5.13) that $c_{1}-c_{2}<0$, so that $\lambda(-1)<0$.

In principle, all $\gamma$-eigenvalues can be determined with the same procedure. (Note that $M^{s}(0,0)-$ $\gamma I$ is invertible as $\gamma$ is by assumption not an eigenvalue of $M^{s}(0,0)$.) The result of such a calculation yields that $\lambda(\gamma)$ is in between $\lambda(-1)<0$ and $\lambda(1)=0$.

If $M^{s}(0,0)$ has an eigenvalue $\gamma^{*} \in \mathbf{S}^{1}$, there is a singularity in equation (5.12), the coefficients $c_{1,2}$ become unbounded as $\gamma \rightarrow \gamma^{*}$. Hence, the above approach, that is based on the expansion (5.1), is no longer valid if $\gamma$ is asymptotically close to $\gamma^{*}$. Nevertheless, Proposition 4.3 (ii) can still be applied, and it can be concluded that there are (uniquely determined) $\gamma$ eigenvalues near 0 for all $\gamma \in \mathbf{S}^{1}$ not too close to $\gamma^{*}$. Moreover, the small spectrum can also still be determined explicitly.

Lemma 5.3 Let $0<\varepsilon \ll \delta \ll 1$ and let $\gamma^{*} \in \mathbf{S}^{1}$ with $\left|\operatorname{Im}\left(\gamma^{*}\right)\right| \gg \delta$. Suppose $\gamma^{*} \in \mathbf{S}^{1}$ is an eigenvalue of $M^{s}(0,0)$.

(i) The $\mathcal{O}\left(\varepsilon^{4}\right)$ spectrum consists of two disjoint parts, $\sigma_{+}^{*} \in\{\lambda \geq 0\}$ and $\sigma_{-}^{*} \in\{\lambda \leq \lambda(-1)<0\}$; for $\gamma \in \mathbf{S}^{1}$ such that $\left|\gamma-\gamma^{*}\right| \gg \delta$ for some $0<\varepsilon \ll \delta \ll 1, \lambda(\gamma) \in \sigma_{+}^{*}$ if $|\arg (\gamma)| \in\left[0,\left|\arg \left(\gamma^{*}\right)\right|\right)$, where $\lambda(1)=0$ is simple, and $\lambda(\gamma) \in \sigma_{-}^{*}$ if $|\arg (\gamma)| \in\left(\left|\arg \left(\gamma^{*}\right)\right|, \pi\right]$ (with $\arg (\gamma) \in[-\pi, \pi]$ ).

(ii) There exist $a \lambda_{-}^{*}<0$ and $a \lambda_{+}^{*}>0$, such that all $\lambda \in\left[\lambda_{-}^{*},-\delta\right)$ and all $\lambda \in\left(\delta, \lambda_{+}^{*}\right]$ are $\mathcal{O}(1)$ $\gamma$-eigenvalues. 
Proof.

(i) The methods by which the small $\gamma$-eigenvalues can be determined - based on (5.4), (5.7), (5.12), (4.18) - are valid for the $\gamma^{\prime}$ 's considered in this Lemma, so that $\lambda(\gamma)$ can be explicitly computed as a function of $\gamma \neq \gamma^{*}$, or, equivalently, as a function of $\arg (\gamma) \in\left[0,\left|\arg \left(\gamma^{*}\right)\right|\right) \cup$ $\left(\left|\arg \left(\gamma^{*}\right)\right|, \pi\right]$. It follows that the function $\lambda=\lambda(\arg (\gamma)) \in \mathbb{R}$ is monotonically increasing with a singularity at $\left|\arg \left(\gamma^{*}\right)\right|$, i.e that $\lambda(\gamma) \geq 0$ for $0 \leq \arg (\gamma)<\left|\arg \left(\gamma^{*}\right)\right|$ and $\lambda(\gamma) \leq \lambda(-1)<0$ for $\pi \geq \arg (\gamma)>\left|\arg \left(\gamma^{*}\right)\right|$.

(ii) Since $\operatorname{Tr}\left[M^{s}(0,0)\right]=2 \operatorname{Re}\left(\gamma^{*}\right)$ (Proposition 3.8), we know that there exist $\lambda_{-}^{*}<0$ and $\lambda_{+}^{*}>0$, such that $-2<\operatorname{Tr}\left[M^{s}(\lambda, 0)\right]<2$ for all $\lambda \in\left[\lambda_{-}^{*}, \lambda_{+}^{*}\right]$. Although the eigenvalues of $M^{s}(\lambda, 0)$ may not provide an accurate approximation of the zeroes of $\mathcal{D}^{s}(\lambda)$ inside $B(0, \delta)$, they do so outside $B(0, \delta)$ (Section 4.3). Hence, in the intervals $\left[\lambda_{-}^{*},-\delta\right)$ and $\left(\delta, \lambda_{+}^{*}\right]$, we have $-2<\operatorname{Tr}\left[M^{s}(\lambda, 0)\right]<2$ which implies that $\mathcal{D}^{s}(\lambda, \gamma)=0$ for $\lambda \in\left[\lambda_{-}^{*},-\delta\right) \cup\left(\delta, \lambda_{+}^{*}\right]$ - Proposition 3.8 .

The unbounded $\lambda$-intervals $\sigma_{+}^{*}$ and $\sigma_{-}^{*}$ are by construction only determined for $\lambda=\mathcal{O}\left(\varepsilon^{4}\right)$. Beyond this magnitude, the small $\mathcal{O}\left(\varepsilon^{4}\right)$ eigenvalues merge with the $\mathcal{O}(1)$ eigenvalues, that - for the same reason - will not be $\mathcal{O}(1)$ for $\gamma$ close to $\gamma^{*}$. Hence, for $\lambda>0$ and for $\lambda<\lambda(-1)=\mathcal{O}\left(\varepsilon^{4}\right)$, there is a (smooth) transition of the $\mathcal{O}\left(\varepsilon^{4}\right)$ eigenvalues into $\mathcal{O}(1)$ eigenvalues. We do not consider the details of this transition here. Neither do we go further into the details of the appearance of the $\gamma^{*}$ singularity into the interval of $\mathcal{O}\left(\varepsilon^{4}\right)$ eigenvalues, i.e. we do not consider the cases that $\gamma^{*}$ is close to \pm 1 .

Remark 5.4 One can also interpret Lemma 5.3 as a result on how the small eigenvalues appear as an $\mathcal{O}\left(\varepsilon^{4}\right)$ 'hole' in the $\mathcal{O}(1) \gamma$-curve of large eigenvalues. Together, Lemmas 5.2 and 5.3 show that the spectrum near $\lambda=0$ behaves as can be expected from the general literature on eigenvalue problems for periodic systems (see for instance [23]). The critical eigenvalue at $\lambda=0$ is either the upper bound of an interval of $\gamma$-eigenvalues (Lemma 5.2), or the lower bound (Lemma 5.3). On the other hand, Lemma 5.3 seems to contradict the results of [27] that are obtained in a more general setting under the assumption that the spatial period of the periodic pattern, $2 L$, is large enough. There, it is shown that the $\gamma$-curve of eigenvalues that include $\lambda=0$ must be a smooth closed curve, i.e. the hole or singularity described in Lemma 5.3 seems to disagree with [27]. However, it will be shown in Section 6.1 that $M^{s}(0,0)$ cannot have eigenvalues $\gamma^{*} \in \mathbf{S}^{1}$ for $L$ (too) large. Hence, for large $L$ the spectrum near $\lambda=0$ is governed by Lemma 5.2, that agrees completely with [27]. For the same reason, the second subcase of Proposition 4.3 - that is also associated with the existence of an eigenvalue $\gamma^{*} \in \mathbf{S}^{1}$ of $M^{s}(0,0)$ - does not contradict the theory of [11] (that is also developed for $L$ large enough).

\section{The stability of fundamental periodic pulse solutions}

In the previous sections, we have developed the machinery by which we can calculate the $\mathcal{O}(1)$ and $\mathcal{O}\left(\varepsilon^{4}\right) \gamma$-eigenvalues of the linearization around a fundamental periodic pulse solution. In this section, we apply these results and determine the stability and instability of the fundamental periodic pulse patterns.

\subsection{Periodic solutions with large spatial periods}

In [11] the stability of periodic patterns that limit on a homoclinic pattern as the period tends to $\infty$ has been studied. It was shown that for large enough spatial periods the $\gamma$-eigenvalues of the periodic eigenvalue problem are close to the spectrum of the linearization around the homoclinic pulse solution. More precisely, it was shown that for every closed curve $K$ that does not intersect the spectrum of the homoclinic pulse, there exists an $L_{c}$ such that for all $L>L_{c}$ 
and for each $\gamma$ on the unit circle the number of $\gamma$-eigenvalues (counting $\gamma$-multiplicity) inside $K$ equals the number of eigenvalues of the homoclinic pulse solution inside $K$.

Our quantitative methods enable us to determine in detail the convergence of the $\gamma$-eigenvalues to the eigenvalues of the homoclinic eigenvalue problem. In this section, we consider $|\Delta|=$ $|\Delta(\lambda, L)|(1.11)$ as a small parameter - note that $\Delta(\lambda, L) \in \mathbb{C}$ for $\lambda \notin \mathbb{R}$ - i.e. we assume that $\varepsilon^{2} L$ is so large that, for all $\lambda \in \mathbb{C}_{r},|\Delta(\lambda)| \ll 1$ with respect to the (artificial) small parameter $\delta$ as introduced in (1.7) and (1.8) - recall that $\Lambda^{s}(\lambda) \neq 0$ for $\lambda \in \mathbb{C}_{e}$. We obtain approximations of both the distance between the curve of $\gamma$-eigenvalues and their homoclinic limit $\lambda_{h}$ and of the length of the curve of $\gamma$-eigenvalues.

The position and multiplicity of the non-trivial $\mathcal{O}(1)$ eigenvalues $\lambda_{h}$, i.e. $\lambda_{h} \neq 0$, of the homoclinic stability problem are known from [3]. In fact, they correspond to zeroes of $t_{11}^{h}(\lambda)=$ $\lim _{L \rightarrow \infty} t_{11}(\lambda)$, see Remark 3.7. Such an eigenvalue can only have multiplicity 2 if two real eigenvalues merge (and leave the real axis as two complex conjugated eigenvalues by changing a parameter). This happens for a special value of $\mu, \mu=\mu_{\text {complex }}\left(\alpha_{1}, \alpha_{2}, \beta_{1}, \beta_{2}\right)$, that can be determined explicitly. Moreover, $\lambda_{h}$ is $\mathcal{O}(\delta)$ close to the essential spectrum associated to the homoclinic stability problem, i.e. $\lambda_{h} \notin \mathbb{C}_{e}$ (Remark $1.4,(1.7)$ ), if $\left|\mu-\mu_{\text {edge }}\right|=\mathcal{O}(\delta)$, where $\mu_{\text {edge }}=\mu_{\text {edge }}\left(\alpha_{1}, \alpha_{2}, \beta_{1}, \beta_{2}\right)$ is a critical value of $\mu$ (that can be determined explicitly) at which a new (homoclinic) eigenvalue appears from the essential spectrum [3]. Eigenvalue $\lambda_{h}$ cannot be $\mathcal{O}(\delta)$ close to an eigenvalue $\lambda_{j}^{r}$ of the fast reduced limit system if $\mu \gg \delta,\left|\alpha_{2}\right| \gg \mathcal{O}(\sqrt{\delta})$ and $\left|\alpha_{1}\right|=\mathcal{O}(1)$ for some $0<\varepsilon \ll \delta \ll 1$ (Remark 4.5). Therefore, we formulate the following assumption,

$$
\mu,\left|\mu-\mu_{\text {complex }}\right|,\left|\mu-\mu_{\text {edge }}\right|,\left|\alpha_{2}\right|,\left|\alpha_{1}\right|=\mathcal{O}(1) \text { with respect to }|\delta|,
$$

so that we know that $\lambda_{h}$ is simple and isolated and that $\lambda_{h}, \lambda(\gamma) \subset \mathbb{C}_{r}$ - where $\lambda(\gamma)$ is the curve that limits on $\lambda_{h}$ in the limit $L \rightarrow \infty$. Note that this assumption can be slightly weakened (Remark 4.5).

Lemma 6.1 Let $0<\varepsilon \ll \delta \ll 1$ and consider $L$ such that $0<|\Delta(\lambda)| \ll 1$ with respect to $\delta$ for all $\lambda \in \mathbb{C}_{r}$. Let assumption $(A)$ be satisfied and let $\lambda_{h} \neq 0$ be an eigenvalue of the linearization (3.3) around a homoclinic pulse solution $\left(U_{h}(\xi), V_{h}(\xi)=\left(U_{p, A}(\xi ; \infty), V_{p, A}(\xi ; \infty)\right)\right.$ (Theorem 1.7). Then there is a zero $\lambda_{p}(L)$ of $t_{11}(\lambda, 0)$ such that $\left|\lambda_{h}-\lambda_{p}(L)\right|=\mathcal{O}\left(\Delta(0)^{2}, \varepsilon\right)$, where $\lambda_{p} \in \mathbb{R}$ if $\lambda_{h} \in \mathbb{R}$. For $\gamma \in \mathbf{S}^{1}$, the $\gamma$-eigenvalues $\lambda(\gamma)$ of (2.2), that limit on $\lambda_{h}$ for $L \rightarrow \infty$, are given by

$$
\lambda(\gamma)=\lambda_{p}-\frac{2 \operatorname{Re}[\gamma]}{\frac{\partial t_{11}}{\partial \lambda}\left(\lambda_{h}, 0\right)} \Delta\left(\lambda_{h}\right)^{2}+\mathcal{O}\left(\left|\Delta\left(\lambda_{h}\right)\right|^{4}, \varepsilon\right)
$$

with $\frac{\partial t_{11}}{\partial \lambda}\left(\lambda_{h}, 0\right) \neq 0$. As a consequence, $\lambda(\gamma)$ is up to $\mathcal{O}\left(\left|\Delta\left(\lambda_{h}\right)\right|^{4}\right)$ corrections approximated by the straight interval connecting $\lambda(1) \in \mathbb{C}$ to $\lambda(-1) \in \mathbb{C}$, and

$$
\begin{gathered}
|\lambda(1)-\lambda(-1)|=4 \frac{\left|\Delta\left(\lambda_{h}\right)\right|^{2}}{\left|\frac{\partial t_{11}}{\partial \lambda}\left(\lambda_{h}, 0\right)\right|}+\mathcal{O}\left(\left|\Delta\left(\lambda_{h}\right)\right|^{4}, \varepsilon\right), \\
\min _{\gamma \in \mathbf{S}^{1}}\left|\lambda_{p}-\lambda(\gamma)\right|=\left|\lambda_{p}-\lambda( \pm i)\right|+\mathcal{O}\left(\left|\Delta\left(\lambda_{h}\right)\right|^{4}, \varepsilon\right)=\mathcal{O}\left(\left|\Delta\left(\lambda_{h}\right)\right|^{4}, \varepsilon\right),
\end{gathered}
$$

so that $\min _{\gamma \in \mathbf{S}^{1}}\left|\lambda_{h}-\lambda(\gamma)\right|=\mathcal{O}\left(\Delta(0)^{2},\left|\Delta\left(\lambda_{h}\right)\right|^{2}, \varepsilon\right)$.

Note that it also follows from (6.1), that the orientation of the $\lambda(\gamma)$-interval, i.e. the argument of $\lambda(1)-\lambda(-1)$, remains constant as a function of $L$ if $\lambda_{h} \in \mathbb{R}$. However, $\arg [\lambda(1)-\lambda(-1)]$ is a periodic function of $L$ if $\lambda_{h} \notin \mathbb{R}$, since $\Delta\left(\lambda_{h}, L\right) \notin \mathbb{R}(1.11)$ in that case. Thus, as $L$ increases, the curve of $\gamma$-eigenvalues approaches $\lambda_{h}$ as a shrinking interval, which rotates around its center $\lambda( \pm i)=\lambda_{p}+\mathcal{O}\left(\left|\Delta\left(\lambda_{h}\right)\right|^{4}\right)$.

Proof. Since $\lambda_{h} \in \mathbb{C}_{r}$ and $|\Delta(\lambda)| \ll 1$ with respect to $\delta$ for all $\lambda_{h} \in \mathbb{C}_{r}$, the results obtained in the previous sections can be applied. Thus, $\lambda(\gamma, \varepsilon)=\lambda(\gamma, 0)+\mathcal{O}(\varepsilon)$, where $\lambda(\gamma, 0)$ is a solution 
of (3.20) - Proposition 3.8. The (leading order) transmission functions $t_{11,22}(\lambda, 0)$ are given by (4.16) and (4.17). $\operatorname{Tr}\left(M^{s}(\lambda, 0)\right)$ can only be $\mathcal{O}(1)$ with respect to $|\Delta|$ if $t_{11}(\lambda, 0)=\mathcal{O}\left(|\Delta|^{2}\right)$ $\left(t_{22}(\lambda, 0)\right.$ cannot be $\mathcal{O}\left(|\Delta|^{-2}\right)$ since that implies that $t_{11}(\lambda, \varepsilon)=\mathcal{O}\left(|\Delta|^{-2}\right)$ as well $\left.(4.16)\right)$. Thus, (3.20) can be rewritten as

$$
t_{11}(\lambda, 0)=2 \Delta(\lambda)^{2} \operatorname{Re}[\gamma]+\mathcal{O}\left(|\Delta|^{4}, \varepsilon\right)
$$

It follows from assumption $(\mathrm{A})$ that $t_{11}^{h}(\lambda, \varepsilon)=0$ (Remark 3.7) and that $\frac{\partial t_{11}^{h}}{\partial \lambda}\left(\lambda_{h}, \varepsilon\right) \neq 0$ (and $\mathcal{O}(1)$ with respect to $\delta)$. In fact,

$$
t_{11}(\lambda, 0)=t_{11}^{h}(\lambda, \varepsilon)\left(\tanh \left(\varepsilon^{2} \sqrt{\mu} L\right)+\mathcal{O}(\varepsilon)\right)=t_{11}^{h}(\lambda, \varepsilon)\left(1+\mathcal{O}\left(|\Delta(0)|^{2}, \varepsilon\right)\right)
$$

(4.16), (4.17), which yields that $t_{11}(\lambda, 0)$ must have a zero $\lambda=\lambda_{p}(L)$ with $\lambda_{p}(L)=\lambda_{h}+$ $\mathcal{O}\left(|\Delta(0)|^{2}, \varepsilon\right)$ and that $\frac{\partial t_{11}}{\partial \lambda}\left(\lambda_{h}, 0\right) \neq 0$ and $\mathcal{O}(1)$ with respect to $\delta$. Thus, (6.1) follows by a direct Taylor expansion of (6.2).

The quantitative control over periodic solutions on large intervals also yields a more qualitative result, that is similar to more general statements in the literature $[11,27]$.

Corollary 6.2 Let $\left(U_{p}(\xi ; L), V_{p}(\xi ; L)\right)$ be a family of fundamental periodic solutions of (1.2) parameterized by its spatial period $2 L$ and let $\left(U_{h}(\xi), V_{h}(\xi)\right)=\left(U_{p}(\xi ; \infty), V_{p}(\xi ; \infty)\right)$ be its homoclinic limit. If $\left(U_{h}(\xi), V_{h}(\xi)\right)$ is (spectrally) stable, respectively unstable, as solution of (1.2) on $\mathbb{R}$, then there is an $L_{c}$ such that $\left(U_{p}(\xi ; L), V_{p}(\xi ; L)\right)$ is (spectrally) stable, resp. unstable, on $\mathbb{R}$ for $L>L_{c}$.

The (in)stability of the homoclinic pattern can be determined by the methods of [3]. For instance, it is shown in [3] that $\left(U_{h}(\xi), V_{h}(\xi)\right)$ is stable as solution of the classical GiererMeinhardt problem (1.2), (1.4) for $\mu>\mu_{\mathrm{Hopf}}=0.36 \ldots$ and that it is destabilized by a Hopf bifurcation as $\mu$ decreases through $\mu_{\mathrm{Hopf}}$. It follows from our analysis that the same is true for periodic patterns $\left(U_{p}(\xi ; L), V_{p}(\xi: L)\right)$ with $L$ large enough.

Proof. The essential spectrum of the stability problem associated to $\left(U_{h}(\xi), V_{h}(\xi)\right)$ is $\subset \mathbb{C} \backslash \mathbb{C}_{e}$. If $\left(U_{h}(\xi), V_{h}(\xi)\right)$ is stable or unstable then all eigenvalues $\lambda_{h} \neq 0$ must be bounded away from the imaginary axis. It follows from [11] that for $L$ large enough all $\mathcal{O}(1) \gamma$-eigenvalues are in the same half plane as their homoclinic limits $\lambda_{h}$. Since

$$
\operatorname{Tr}\left(M^{s}(0,0)\right)=\frac{1}{\Delta(0)^{2}} \frac{D}{\beta_{2}-1}+\mathcal{O}(1) \gg 1
$$

(4.18), (1.3) for $L \gg 1$, it follows that $M^{s}(0,0)$ cannot have eigenvalues $\gamma \in \mathbf{S}^{1}$, thus all nontranslational small eigenvalues are in the stable half plane for $L$ large enough (Lemma 5.2).

Remark 6.3 By (the proof of) Lemma 5.2, in combination with the information on $U_{0}$ and $U_{\min }$ (Section 1.1), it follows that the asymptotic length of the interval of small $\gamma$-eigenvalues is $\mathcal{O}\left(\varepsilon^{4} e^{-2 \varepsilon^{2} \sqrt{\mu} L}\right)$ for large $L$.

\subsection{The destabilization of fundamental periodic pulse solutions}

As $L$ decreases the curves of $\gamma$-eigenvalues, i.e. the solutions $\lambda(\gamma)$ of $(1.10)$, increase in length and move away from the homoclinic eigenvalues. Therefore, it is possible that (part of) a curve that is in the stable half plane for large $L$, crosses the imaginary axis into the unstable half plane as $L$ decreases. As in the homoclinic case [3], whether (and when) this occurs can only be determined by direct evaluation of (1.10). On the other hand, it is possible to analyze whether $M^{s}(0,0)$ has an eigenvalue in $\mathbf{S}^{1}$. Recall, that if $M^{s}(0,0)$ has an eigenvalue in $\mathbf{S}^{1}$, there exists unstable $\mathcal{O}(1)$ spectrum - Lemma 5.3. 
Lemma 6.4 The matrix $M^{s}(0,0)$ has an eigenvalue $\gamma=-1 \in \mathbf{S}^{1}$, i.e. $\lambda(-1)=0$, at $L=$ $L_{\mathrm{SN}}(\mu)$. The eigenvalues $\rho, 1 / \rho$ of $M^{s}(0,0)$ are not in $\mathbf{S}^{1}$ if $L>L_{\mathrm{SN}}(\mu)$. If $L<L_{\mathrm{SN}}, \rho, 1 / \rho \in$ $\mathbf{S}^{1}$, and for every $\gamma \in \mathbf{S}^{1} \backslash\{1\}$, there exists a uniquely determined $L_{c}=L_{c}(\gamma)$ such that $\gamma$ is an eigenvalue of $M^{s}(0,0)$ at $L=L_{c} ; 1$ is never an eigenvalue of $M^{s}(0,0)$.

Proof. The eigenvalues $\rho$ of $M^{s}(0,0)$ are (at leading order) determined by $T^{s}(0, L)=$ $\operatorname{Tr}\left(M^{s}(0,0)\right)=\rho+1 / \rho(1.10)$. Here, (1.10) can be written as

$$
f\left(\Delta_{0}, \gamma\right)=\alpha_{1}-\alpha_{2} \beta_{1} \mathcal{R}\left(0 ; \beta_{1}, \beta_{2}\right)=1+\frac{D}{\beta_{2}-1}>1
$$

(4.14), where

$$
f\left(\Delta_{0}, \gamma\right)=\frac{\left(\Delta_{0}^{2}-\gamma\right)\left(\Delta_{0}^{2}-\bar{\gamma}\right)}{\left(\Delta_{0}^{2}-1\right)^{2}}=\frac{\Delta_{0}^{4}-2 \Delta_{0}^{2} \operatorname{Re}[\gamma]+1}{\left(\Delta_{0}^{2}-1\right)^{2}}
$$

since

$$
\tanh \left(\varepsilon^{2} \sqrt{\mu} L\right)=\frac{e^{\varepsilon^{2} \sqrt{\mu} L}-e^{-\varepsilon^{2} \sqrt{\mu} L}}{e^{\varepsilon^{2} \sqrt{\mu} L}+e^{-\varepsilon^{2} \sqrt{\mu} L}}=\frac{\frac{1}{\Delta_{0}}-\Delta_{0}}{\frac{1}{\Delta_{0}}+\Delta_{0}}=\frac{1-\Delta_{0}^{2}}{1+\Delta_{0}^{2}}
$$

with $\Delta_{0}=\Delta(0)$. It can be checked that $f\left(\Delta_{0}, \gamma\right)$ is monotonous as function of $\Delta_{0} \in(0,1)$. Clearly $f\left(\Delta_{0}, 1\right)=1$ for all $\Delta_{0}$, so that (6.4) does not have a solution for $\gamma=1$. On the other hand,

$$
f\left(\Delta_{0},-1\right)=\frac{\left(\Delta_{0}^{2}+1\right)^{2}}{\left(\Delta_{0}^{2}-1\right)^{2}}=\tanh ^{-2}\left(\varepsilon^{2} \sqrt{\mu} L\right)=\left(\frac{U_{\mathrm{hom}}}{U_{0}}\right)^{\frac{2 D}{\beta_{2}-1}}
$$

by (1.25). This yields by (1.24) that (6.4) is satisfied at the saddle-node bifurcation. Thus $M^{s}(0,0)$ has an eigenvalue -1 if $L=L_{\mathrm{SN}}(\mu)$. Since $f\left(\Delta_{0}, \gamma\right)<f\left(\Delta_{0},-1\right)$ for all $\gamma \neq-1$ and $\Delta_{0} \in(0,1)$, it follows that $f\left(\Delta_{0}^{\mathrm{SN}}, \gamma\right)<1+\frac{D}{\beta_{2}-1}$ if $\gamma \neq-1$. For $\Delta_{0}<\Delta_{0}^{\mathrm{SN}}=\Delta_{0}\left(L_{\mathrm{SN}}(\mu)\right)$ (or equivalently if $\left.L>L_{\mathrm{SN}}\right), f\left(\Delta_{0}, \gamma\right) \leq f\left(\Delta_{0},-1\right)<1+\frac{D}{\beta_{2}-1}$, so that (6.4) cannot be satisfied, i.e. $M^{s}(0,0)$ cannot have eigenvalues $\in \mathbf{S}^{1}$ for $L>L_{\mathrm{SN}}(\mu)$. Moreover, $\lim _{\Delta_{0} \rightarrow 1} f\left(\Delta_{0}, \gamma\right)=+\infty$ for all $\gamma \neq 1$, so that there exists a $\Delta_{0}=\Delta_{0}(L)>\Delta_{0}^{\mathrm{SN}}$ for every $\gamma \neq \pm 1$ such that (6.4) is satisfied, i.e. there is an $L_{c}$ such that $M^{s}(0,0)$ has eigenvalues $\gamma, \bar{\gamma} \in \mathbf{S}^{1}$ at $L=L_{c}$.

Remark 6.5 For a given $\gamma \in \mathbf{S}^{1}$, the critical period $2 L_{c}$ of the fundamental pattern at which $M^{s}(0,0)$ has an eigenvalue $\gamma$, can be explicitly determined through (6.4),

$$
L_{c}(\gamma)=\frac{1}{\varepsilon^{2}} \frac{1}{2 \sqrt{\mu}} \operatorname{arccosh}\left(1+(1-\operatorname{Re}[\gamma]) \frac{\beta_{2}-1}{D}\right) .
$$

Note, that $L_{c}(\gamma)<L_{c}(-1)=L_{S N}($ see $(1.27)$ and recall that $2 \operatorname{arccosh} \sqrt{Y}=\operatorname{arccosh}(2 Y-1))$.

Corollary 6.6 Let $\left(U_{p}(\xi ; L), V_{p}(\xi ; L)\right)$ be a fundamental periodic solution of (1.2). If it is of type A, i.e. if $L>L_{S N}(\mu)$, then there exists a $\tilde{\delta}>0$, such that there is no $\mathcal{O}(1)$ spectrum in the ball $B(0, \tilde{\delta})(1.9)$; all small non-translational eigenvalues are negative and contained in $B(0, \tilde{\delta})$. If $\left(U_{p}(\xi ; L), V_{p}(\xi ; L)\right)$ is of type $B$, i.e. if $L<L_{S N}(\mu)$, then are positive real $\mathcal{O}(1) \gamma$-eigenvalues; the small eigenvalues may be either positive or negative. Hence, the periodic patterns of type $B$ are always unstable as solutions on $\mathbb{R}$, their most unstable $\gamma$-eigenvalues are always of $\mathcal{O}(1)$.

This corollary might seem to contradict the results obtained in $[17,29]$ - in which it is shown that the destabilization at the saddle-node bifurcation is induced by the $\mathcal{O}\left(\varepsilon^{4}\right)$ eigenvalues. However, this is not the case, as is explained in Section 8.2, see especially Corollary 8.4.

Of course, it is not claimed in Corollary 6.6 that all periodic patterns of type A are stable. As was already noted, an A-pattern may destabilize by a Hopf bifurcation (see also [17, 29]). Though (1.10) gives an expression for the $\gamma$-eigenvalues, it gives no direct analytical insight in 
their positions. Therefore, we give a number of prototypical examples of the development of the curves of $\mathcal{O}(1) \gamma$-eigenvalues in the next section for the classical Gierer-Meinhardt case (1.4). All calculations of $\gamma$-eigenvalues are based on evaluating (1.10) with the help of Mathematica. Note that $\beta_{2}=2$, so these calculations do not involve hypergeometric functions (Remark 4.2).

Proof. If $L>L_{S N}(\mu)$, Lemma 6.4 yields that $\operatorname{Tr}\left[M^{s}(0,0)\right] \notin[-2,2]$, so that it immediately follows that there is a $\tilde{\delta}>\delta>0$ (with $\delta$ as in (1.7)) such that there are no $\mathcal{O}(1) \gamma$-eigenvalues inside $B(0, \tilde{\delta})$ (Proposition 3.8, Section 4.3). If $L<L_{S N}(\mu)$, there exists an eigenvalue $\gamma^{*}$ of $M^{s}(0,0)$ in $\mathbf{S}^{1}$. Then by Lemma 5.3 , there exists an interval of positive unstable $\mathcal{O}(1) \gamma$ eigenvalues. Thus, (2.2) has positive $\gamma$-eigenvalues if $L<L_{S N}(\mu)$, i.e. the periodic B-patterns are unstable. The statements on the small spectrum are based on Lemmas 5.2 and 5.3.

Remark 6.7 Lemma 6.4 only shows that $\lambda=0$ is an $\mathcal{O}(1)$-1-eigenvalue to leading order (in $\varepsilon$ ) if $L=L_{S N}(\mu)$. However, it can be shown in various ways that $\lambda=0$ indeed exactly is an -1-eigenvalue at the saddle-node bifurcation. In fact, the bifurcation at $L=L_{S N}(\mu)$ can be seen as an period doubling bifurcation, since the fundamental patterns of A- and B-type bifurcate at $L_{S N}(\mu)$ into the periodic AB-patterns, with twice the period of the original A-, B-patterns. The structure of the marginally unstable -1-eigenvalue also indicates that the A-, B-patterns bifurcate into an AB-pattern at $L=L_{S N}(\mu)$.

\subsection{The classical Gierer-Meinhardt equation}

In this section we apply the theory developed in the preceding sections (i.e. in essence Theorem 1.1) to the special case of the classical Gierer-Meinhardt equation (1.2) with (1.4). We consider as examples two special cases $\left(\mu=\frac{1}{2}\right.$ and $\left.\mu=1\right)$, and consider the period $2 L$ of the fundamental periodic pattern $\left(U_{p, A}(\xi ; L), V_{p, A}(\xi ; L)\right)$ of A-type as bifurcation parameter.

Theorem 6.8 Let $0<\varepsilon \ll 1$ and consider $\mu=1$. Let $\left(U_{p, A}(\xi ; L), V_{p, A}(\xi ; L)\right)$ be a fundamental periodic pattern of (1.2) with (1.4) - Theorem 1.7. Then, all fundamental patterns of type $A$ are spectrally stable as solutions of (1.2) on $\mathbb{R}$, i.e. $\left(U_{p, A}(\xi ; L), V_{p, A}(\xi ; L)\right)$ is stable for $L>$ $\frac{1}{\varepsilon^{2}} \tilde{L}_{\mathrm{SN}}=\frac{1}{\varepsilon^{2}}(|\log [\sqrt{2}-1]|+\mathcal{O}(\varepsilon)) . A s \varepsilon^{2} L$ decreases through $\tilde{L}_{\mathrm{SN}}$, then $\left(U_{p, A}(\xi ; L), V_{p, A}(\xi ; L)\right)$ is destabilized by the saddle-node/period-doubling bifurcation associated to the real $\mathcal{O}(1) \gamma-$ eigenvalues $\lambda(\gamma)$; the first eigenvalue to cross the imaginary axis is the-1-eigenvalue.

Theorem 6.9 Let $0<\varepsilon \ll 1$ and consider $\mu=\frac{1}{2}$. Let $\left(U_{p, A}(\xi ; L), V_{p, A}(\xi ; L)\right)$ be a fundamental periodic pattern of 1.2) with (1.4). Then, $\left(U_{p, A}(\xi ; L), V_{p, A}(\xi ; L)\right)$ is spectrally stable as solution of (1.2) on $\mathbb{R}$ for $L>\frac{1}{\varepsilon^{2}} \tilde{L}_{\mathrm{Hopf}}=\frac{2.0 \ldots}{\varepsilon^{2}}\left(>L_{\mathrm{SN}}\right)$. As $\varepsilon^{2} L$ decreases through $\tilde{L}_{\mathrm{Hopf}}$, then $\left(U_{p, A}(\xi ; L), V_{p, A}(\xi ; L)\right)$ is destabilized by two complex conjugate branches of $\mathcal{O}(1) \gamma$-eigenvalues $\lambda(\gamma)$; the first eigenvalues to cross the imaginary axis are 1-eigenvalues.

The proofs of Theorems 6.8 and 6.9 can be obtained by direct evaluation of (1.10), see especially Section 4.1 and Remark 4.2. Note that both theorems confirm the result obtained in [3] on the stability of the homoclinic patterns $\left(U_{h}(\xi), V_{h}(\xi)\right)=\left(U_{p, A}(\xi ; \infty), V_{p, A}(\xi ; \infty)\right)$ (Theorem 5.11 in [3]), that corresponds to the limits $L \rightarrow \infty$ in Theorems 6.8 and 6.9. Of course, the analytical information on $\tilde{L}_{\mathrm{SN}}$ is based on Corollary 6.6 and (1.27). Note also that it follows from Lemma 5.2 that we do not need to pay attention to the small $\mathcal{O}\left(\varepsilon^{4}\right)$ eigenvalues (for patterns on $\mathbb{R}$, this is not the case for periodic patterns on bounded intervals - see Section 8).

The outcome of the Mathematica-assisted calculations is presented graphically in the Figures 3-6. In these Figures, $\tilde{L}=\varepsilon^{2} L$ has been chosen as natural bifurcation parameter. Note that these Figures supply more information than described in Theorems 6.8 and 6.9. For instance, it follows from Figure 3 that the complex $\gamma$-eigenvalues do cross the imaginary axis for $\mu=1$, 

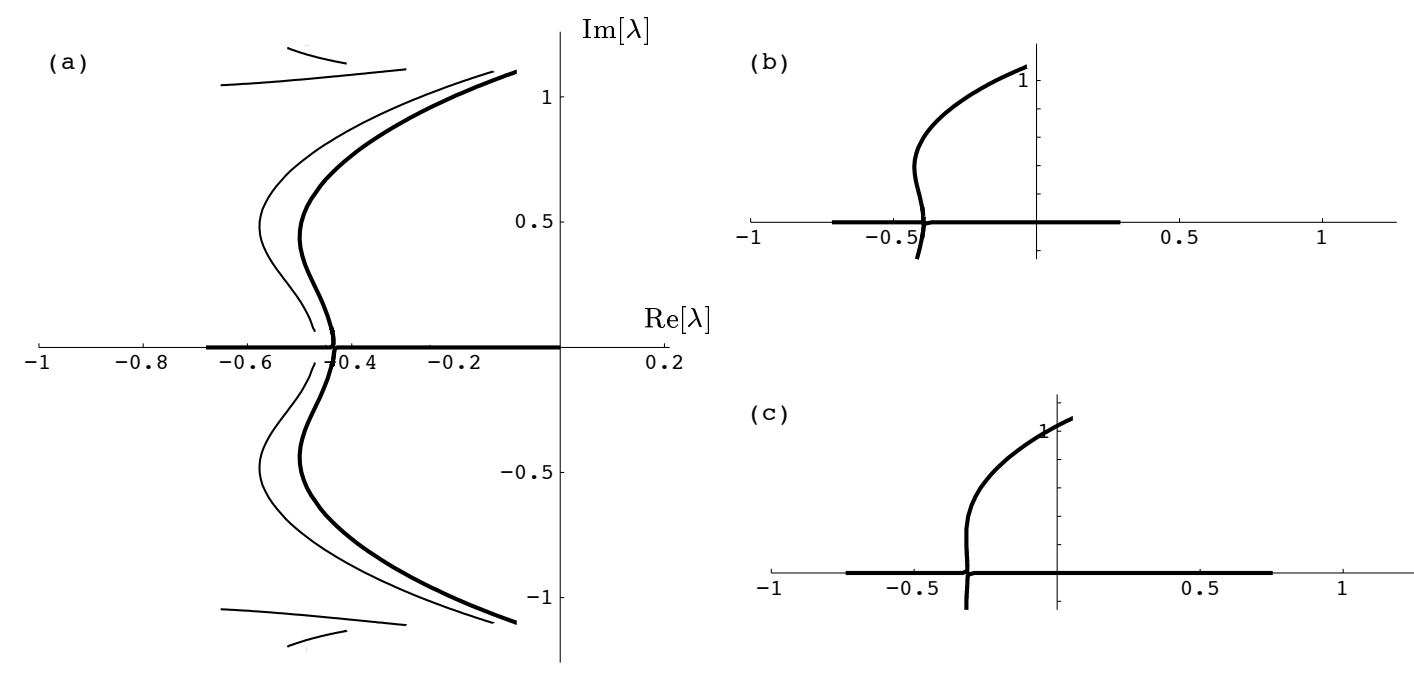

Figure 3: The leading order representations of the curves $\left\{\lambda(\gamma) ; \gamma \in \mathbf{S}^{1}\right\} \subset \mathbb{C}_{e}$ of $\mathcal{O}(1) \gamma$ eigenvalues as given by Theorem 1.1 for the classical Gierer-Meinhardt case (1.4) with $\mu=1.0$ and $\varepsilon=0.1$, for various values of $\tilde{L}=\varepsilon^{2} L$. In (a) $\tilde{L}=5,2,1.5,1$ and $\tilde{L}=\tilde{L}_{S N}=|\log [\sqrt{2}-1]|=$ $0.88 \ldots$ (Theorem 6.8). It is shown in (b), $\tilde{L}=0.75$, and (c) $\tilde{L}=0.5$, that also the complex valued $\gamma$-eigenvalues cross through the imaginary axis (for $\tilde{L}<\tilde{L}_{\mathrm{SN}}$ ). Note that the $\mathcal{O}\left(\varepsilon^{4}\right)$ 'hole' of small $\gamma$-eigenvalues near 0 (Lemma 5.3) is not represented in (b) and (c), and that part of the spectrum with negative imaginary part is not plotted in (b) and (c).

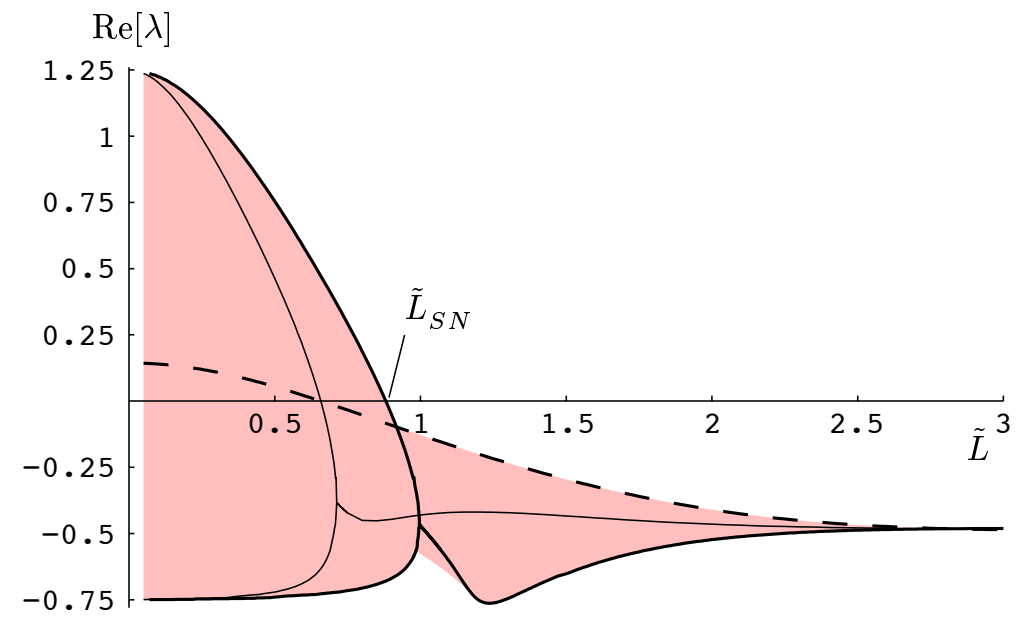

Figure 4: The real part of (all possible) $\mathcal{O}(1) \gamma$-eigenfunctions as function of $\tilde{L}=\varepsilon^{2} L$ for the classical Gierer-Meinhardt case with $\mu=1.0$ and $\varepsilon=0.1$. The dashed line denotes (the real part of) the 1-eigenvalues, the thick line the -1 -eigenvalues, the thin line the $\pm i$-eigenvalues; all other $\mathcal{O}(1) \gamma$-eigenvalues are in the grey area.

this only occurs after the pattern is destabilized by the saddle-node bifurcation. In Figure 5 it is shown that the branch point of two combined curves of $\gamma$-eigenvalues may pass through the imaginary axis, and thus through the small spectrum. The precise interaction between a branch point and the small spectrum is a subtle higher order effect that has not been considered in the analysis of Section 5. Finally, we note that the spectrum associated to the B-patterns $\left(U_{p, B}(\xi ; L), V_{p, B}(\xi ; L)\right)$ seems to cover large parts of the real axis $\left(\lambda<\lambda_{0}^{r}=\frac{5}{4}\right.$, Lemma 3.3) as $L$ decreases. This observation can be proven with the methods developed in this paper (see also Remark 4.4). 

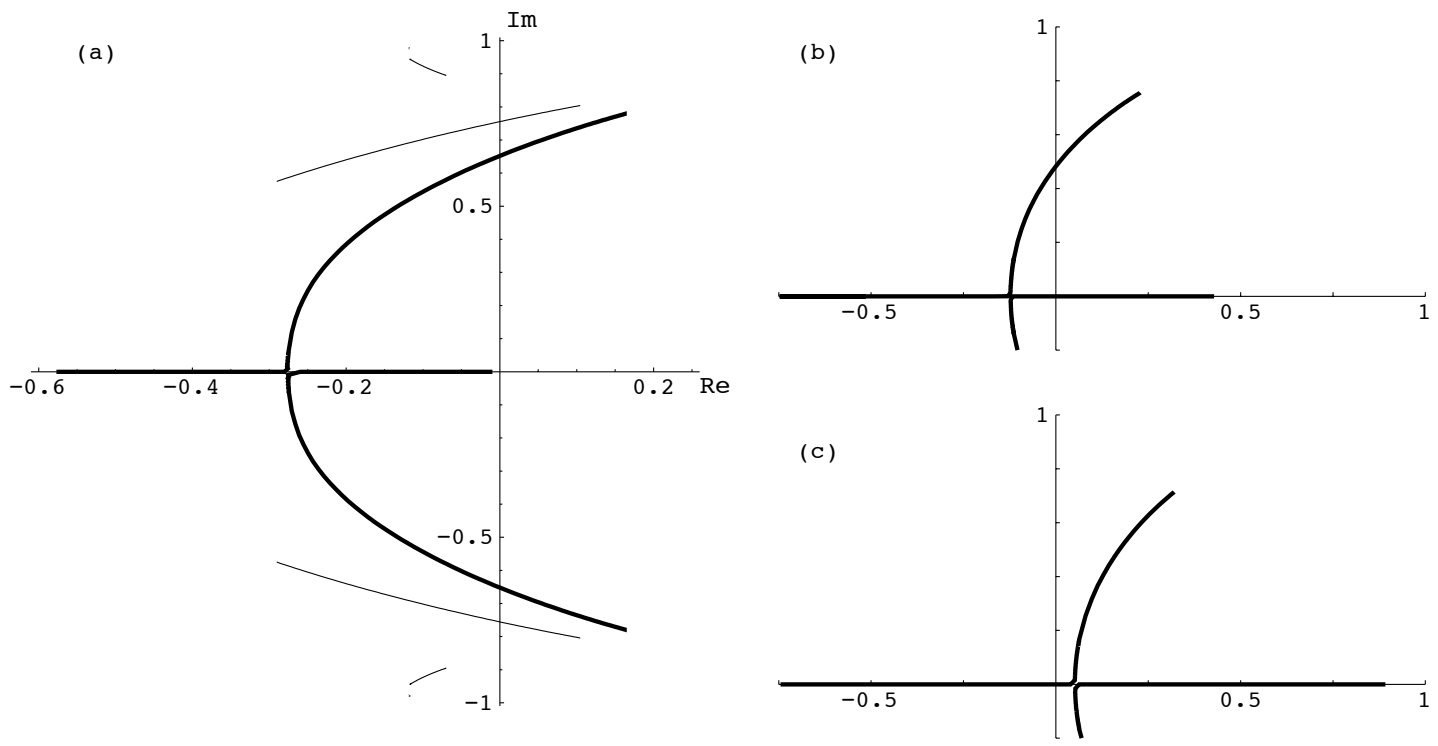

Figure 5: The leading order representations of the curves $\left\{\lambda(\gamma) ; \gamma \in \mathbf{S}^{1}\right\} \subset \mathbb{C}_{e}$ of $\mathcal{O}(1) \gamma$ eigenvalues as given by Theorem 1.1 for the classical Gierer-Meinhardt case with $\mu=0.5$ and $\varepsilon=0.1 ; \tilde{L}=\varepsilon^{2} L=5,2.5,1.5,1.25$ in (a). At $\tilde{L}=\tilde{L}_{\mathrm{Hopf}}=2.0 \ldots\left(>\tilde{L}_{\mathrm{SN}}=\sqrt{2}|\log [\sqrt{2}-1]|=\right.$ $1.24 \ldots(1.27)$ ), two complex conjugate $\lambda(1)$ 's cross the imaginary axis (Theorem 6.9). A branch point crosses the imaginary axis (and the small spectrum) as $\tilde{L}$ decreases from 1 (b) to 0.6 (c).

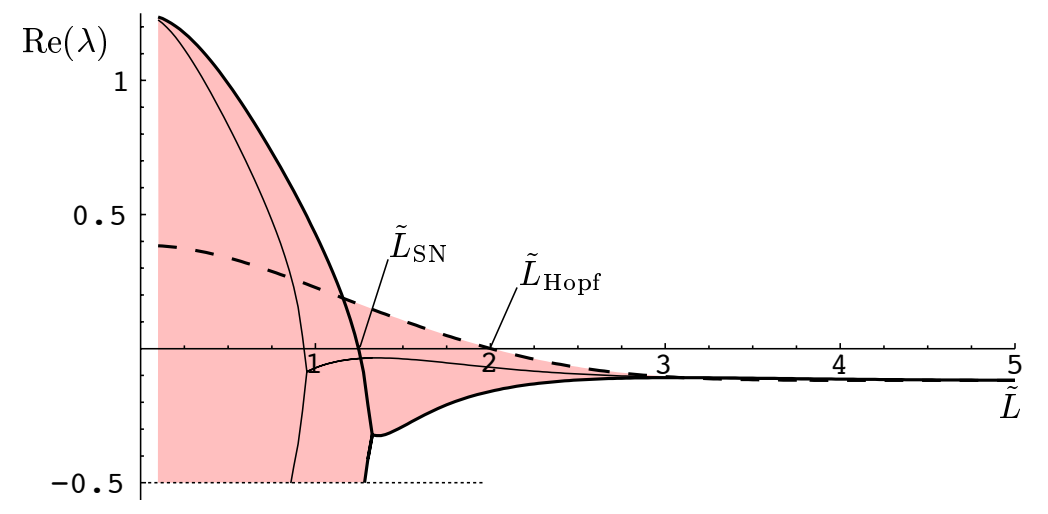

Figure 6: The real part of (all possible) $\mathcal{O}(1) \gamma$-eigenfunctions as function of $\tilde{L}$ for the classical Gierer-Meinhardt case with $\mu=0.5$ and $\varepsilon=0.1$. The dashed line denotes the 1-eigenvalues, the thick line the -1 -eigenvalues, the thin line the $\pm i$-eigenvalues; all other $\mathcal{O}(1) \gamma$-eigenvalues are in the grey area. Note that the region of $\gamma$-eigenvalues is bounded from below by $\mathbb{C} \backslash \mathbb{C}_{e}$, which extends up to $\lambda=\mu(+\delta)(1.7)$.

In Figure 7, the critical (destabilizing) values of $\tilde{L}$ as function of $\mu$ are presented. Hence, this Figure gives the values of $L$ and $\mu$ for which a fundamental periodic pattern $\left(U_{p, A}(\xi ; L), V_{p, A}(\xi ; L)\right)$ is (spectrally) stable. Moreover, it can be concluded from Figure 7 that $\left(U_{p, A}(\xi ; L), V_{p, A}(\xi ; L)\right)$ is destabilized by the saddle-node bifurcation for $\mu>\mu_{\mathrm{SN}-\mathrm{Hopf}}=0.84 \ldots$, i.e. the scenario described by Theorem 6.8, and by the Hopf bifurcation scenario of Theorem 6.9 for $\mu<$ $\mu_{\mathrm{SN}-\mathrm{Hopf}}=0.84 \ldots$. Note that there is a vertical asymptote at $\mu=\mu_{\mathrm{Hopf}}=0.36 \ldots$, the Hopf bifurcation value of $\mu$ that (de)stabilizes the homoclinic pulse pattern $\left(U_{h}(\xi), V_{h}(\xi)\right)=$ $\left(U_{p, A}(\xi ; \infty), V_{p, A}(\xi ; \infty)\right)[3]$. Although this is not clear from the Figure, the lines representing the 1 - and the -1 -eigenvalues have countably many intersections near $\mu=\mu_{\text {Hopf }}$ as $\tilde{L} \rightarrow \infty$, 


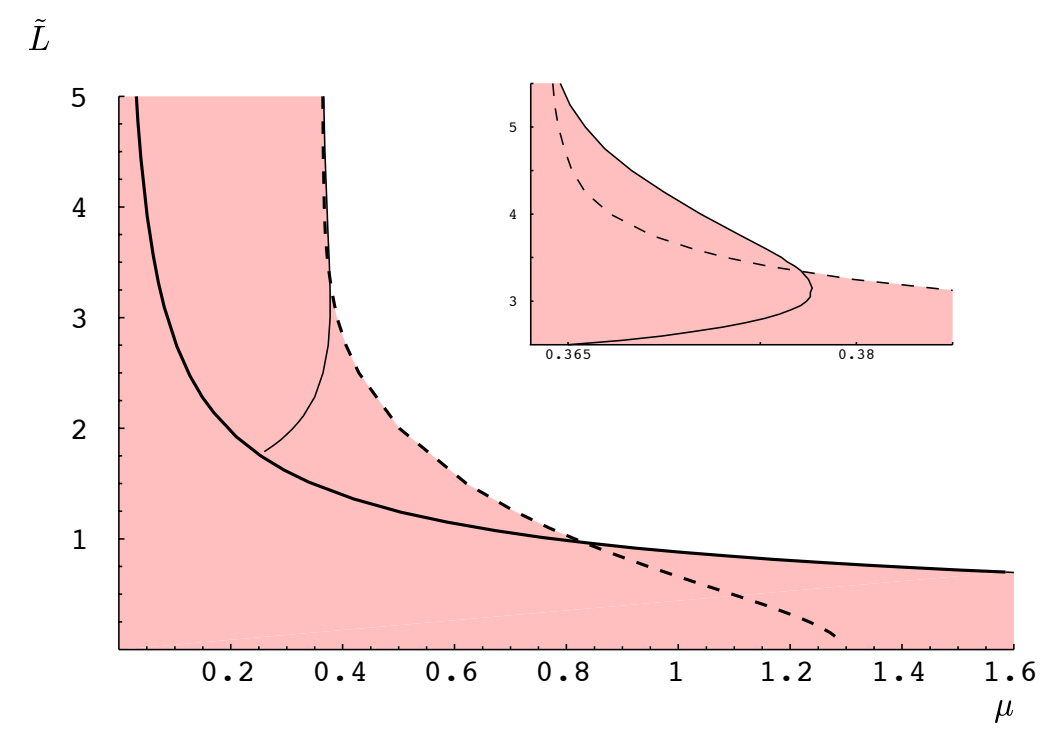

Figure 7: The stability region of a fundamental $2 L$-periodic pulse solution $\left(U_{p, A}(\xi ; L), V_{p, A}(\xi ; L)\right)$ of the classical Gierer-Meinhardt equation (1.2) with (1.4) in $(\mu, \tilde{L})$-space. In the shaded region the periodic pulse solution is unstable. The curves denote the $(\mu, \tilde{L})$-values where a complex conjugate pair of 1-eigenvalue (dashed) or of -1-eigenvalue (solid) crosses the imaginary axis. The bold line represents $\left(\mu, \tilde{L}_{\mathrm{SN}}\right)$-points where a real -1-eigenvalue passes through the origin (1.27). The inset shows a blow-up for large $\tilde{L}$ near $\mu=\mu_{\mathrm{Hopf}}$, the $\mu$-value of Hopf-bifurcation of the homoclinic limit pattern.

the first of these is shown in the inset of Figure 7. This is due to the rotation of the (shrinking) interval of $\gamma$-eigenvalues $\lambda(\gamma)$ as $L \rightarrow \infty$ described in Lemma 6.1. Thus, for $\mu$ close to $\mu_{\mathrm{Hopf}}$, $\left(U_{p, A}(\xi ; L), V_{p, A}(\xi ; L)\right)$ may either be destabilized by a complex conjugate pair of $\gamma(1)$ - or of $\gamma(-1)$-eigenvalues (see the inset of Figure 7 ). This behavior also implies that a pulse pattern $\left(U_{p}(\xi ; L), V_{p}(\xi ; L)\right)$ may change its stability a (finite) number of times as function of $L$ if $\mu$ is chosen close enough to $\mu_{\mathrm{Hopf}}$ (and $\mu<\mu_{\mathrm{Hopf}}$, so that $\left(U_{p, A}(\xi ; L), V_{p, A}(\xi ; L)\right.$ ) is stable for $L$ large enough - Corollary 6.2).

\section{The periodic orbits of AB-type}

In this section, we study the linear stability of the periodic solution $\left(U_{p, A B}(\xi ; L), V_{p, A B}(\xi ; L)\right)$ of AB type with period $2 L$ (Theorem 1.8). The general results from Section 2 apply to this case. For reference, we recall the main ingredients. The linearization around $\left(U_{p, A B}, V_{p, A B}\right)$ is given by

$$
\dot{\phi}(\xi)=A_{p, A B}(\xi ; \lambda) \phi(\xi)
$$

where $A_{p, A B}(\xi ; \lambda)=A_{p}(\xi ; \lambda)(2.3)$ with $U_{p, A B}$ and $V_{p, A B}$ substituted for $U_{p}$ and $V_{p}$. Again, the spectrum consists entirely of $\gamma$-eigenvalues, so the study of the eigenvalue problem can be restricted to one period. For technical reasons, this interval will be chosen different than in the stability analysis of the fundamental periodic orbits. Using the translational invariance, we assume that $U_{p, A B}(\xi)$ has a minimum in $\xi=0$. The first pulse, which we assume to be of A-type, is then located at $\xi_{A}=L_{A}=L_{A}(\nu)$ (1.26). The second pulse is then located at $\xi_{B}=2 L_{A}+L_{B}=2 L-L_{B}(\nu)$. The eigenvalue problem is studied on the interval $[0,2 L]$; hence we define the associated monodromy matrix $M_{A B}$ by

$$
\Psi_{A B}(2 L)=\Psi_{A B}(0) M_{A B}(\lambda, \varepsilon),
$$


where $\Psi_{A B}(\xi)$ is a fundamental matrix solution of (7.1). The Evans function associated to (7.1) is defined by

$$
\mathcal{D}_{A B}(\lambda, \gamma)=\operatorname{det}\left[M_{A B}(\lambda, \varepsilon)-\gamma I\right] .
$$

It follows from the existence analysis ([6], Theorem 1.8) that on the interval $\left[0,2 L_{A}\right]$, the components $U_{p, A B}(\xi)$ and $V_{p, A B}(\xi)$ are uniformly exponentially close to $U_{p}(\xi)$ and $V_{p}(\xi)$, the $U, V$ component of the fundamental periodic solution of A-type with period $2 L_{A}>2 L_{S N}(\mu)$ that has a pulse in $\xi_{A}$. Therefore, the matrix $A_{p, A B}(\xi)$ is exponentially close to $A_{p, A}(\xi)$ for $\xi \in\left[0,2 L_{A}\right]$, where $A_{p, A}(\xi)$ is the matrix obtained in the linearization around $\left(U_{p, A}(\xi), V_{p, A}(\xi)\right)$. Similarly, for $\xi \in\left[2 L_{A}, 2 L\right]$, the matrix $A_{p, A B}(\xi)$ is uniformly exponentially close to $A_{p, B}(\xi)$, the matrix obtained from the linearization around the fundamental periodic solution $\left(U_{p, B}(\xi), V_{p, B}(\xi)\right)$ with period $2 L_{B}$. In the analysis in this section, we will divide the interval $[0,2 L]$ in the subintervals $\left[0,2 L_{A}\right]$ and $\left[2 L_{A}, 2 L\right]$ and approximate the solutions on these intervals by the solutions to the eigenvalue problem of the fundamental periodic solutions.

As in the analysis of the fundamental periodic patterns, we will determine 4 independent solutions to (7.1), such that the fundamental matrix $M_{A B}(\lambda)$ spanned by these solutions has a form that facilitates the evaluation of $\mathcal{D}_{A B}$. Since $A_{p, A B}(\xi ; \lambda)$ is exponentially close to the limit matrix $A_{\infty}(\lambda)$ (3.1) outside the pulse regions, solutions to (7.1) behave to leading order as combinations of $E_{ \pm}^{f} e^{ \pm \Lambda^{f} \xi}$ and $E_{ \pm}^{s} e^{ \pm \Lambda^{s} \xi}$ in these outer regions. Hence, we again look for a splitting in fast solutions $\phi_{1,2}^{f}$ and slow solutions $\phi_{1,2}^{s}$. The analysis is - by construction - very similar to that of Sections 3,4 and 5, therefore we will refrain from giving the full analytical details.

We define the pulse regions around the pulses by

$$
\mathcal{P}_{A}=\left(\xi_{A}-\frac{1}{\sqrt{\varepsilon}}, \xi_{A}+\frac{1}{\sqrt{\varepsilon}}\right) \quad \text { and } \quad \mathcal{P}_{B}=\left(\xi_{B}-\frac{1}{\sqrt{\varepsilon}}, \xi_{B}+\frac{1}{\sqrt{\varepsilon}}\right)
$$

and the outer regions by

$$
\mathcal{I}_{0}=\left[0, \xi_{A}-\frac{1}{\sqrt{\varepsilon}}\right], \quad \mathcal{I}_{1}=\left[\xi_{A}+\frac{1}{\sqrt{\varepsilon}}, \xi_{B}-\frac{1}{\sqrt{\varepsilon}}\right] \quad \text { and } \quad \mathcal{I}_{2}=\left[\xi_{B}+\frac{1}{\sqrt{\varepsilon}}, 2 L\right] .
$$

Remark 7.1 For $L=L_{S N}$, the A- and B-pulses of the AB-periodic orbit are actually the same. Hence, the AB-periodic solution with period $2 L$ consists of 2 copies of a fundamental periodic solution with half the period, i.e. a period $L$. This implies that the $\gamma$-eigenvalues for the linearization around $\left(U_{p, A B}, V_{p, A B}\right)$ are the same as the $\gamma$-eigenvalues for the linearization around $\left(U_{A}, V_{A}\right)$, though the Floquet multiplier of the corresponding eigenfunctions is different. Hence, the spectrum is the same, but the parameterization of the curves of $\gamma$ eigenvalues is different (Remark 2.6). Since a solution $\phi^{*}$ that satisfies $\phi^{*}(\xi+L)=\gamma \phi^{*}(\xi)$, also satisfies $\phi^{*}(\xi+2 L)=\gamma^{2} \phi^{*}(\xi)$, the Floquet multiplier of the eigenfunctions runs over the unit circle twice as fast. Therefore, we consider one curve of $\gamma$-eigenvalues of the linearization around $\left(U_{p, A}(\xi), V_{p, A}(\xi)\right)$ as two curves of $\gamma$-eigenvalues of the linearization around $\left(U_{p, A B}(\xi), V_{p, A B}(\xi)\right)$. Since $\lambda=0$ is an $\mathcal{O}(1)$-1-eigenvalue for the linearization around $\left(U_{p, A}(\xi), V_{p, A}(\xi)\right)$ at $L=L_{S N}, \lambda=0$ is an $\mathcal{O}(1)$ 1-eigenvalue for the linearization around $\left(U_{p, A B}(\xi), V_{p, A B}(\xi)\right)$ at $L=L_{S N}$ (Lemma 6.4, see also Figure $8(\mathrm{a})$ ). Actually, $\lambda=0$ is a 1-eigenvalue of $\gamma$-multiplicity 2, since the derivative of the AB solution is also a 1-eigenvalue.

\subsection{The $\mathcal{O}(1) \gamma$-eigenvalues}

We define $\phi_{1}^{f}$ as the solution to (7.1), that satisfies $\phi_{1}^{f}(0)=e^{-\Lambda^{f} L} E_{+}^{f}$. In general, a solution grows as the fastest growing eigenfunction $E_{+}^{f} e^{\Lambda^{f} \xi}$ on every outer region. Since the total length of the outer regions is (to leading order) $2 L$, we expect that there exists a transmission function $t_{A B}^{f}(\lambda)$, such that $e^{-\Lambda^{f} L} \phi_{1}^{f}(2 L)=t_{A B}^{f} E_{+}^{f}+$ exponentially small terms. We make this more 
precise by first tracking $\phi_{1}^{f}(\xi)$ over the interval $\left[0,2 L_{A}\right]$ and then over $\left[2 L_{A}, 2 L\right]$. On $\left[0,2 L_{A}\right]$, the matrix $A_{p, A B}(\xi)$ is exponentially close to $A_{p, A}(\xi)$, thus the solution $\phi_{1}^{f}(\xi)$ behaves to leading order as the solution $\phi_{A, 1}^{f}(\xi)$ to $(2.2)$, with $A_{p}(\xi)=A_{p, A}(\xi)$, that was defined in Lemma 3.2. Hence, there exists a transmission function $t_{A}^{f}(\lambda)$, such that up to exponentially small terms,

$$
\phi_{1}^{f}\left(2 L_{A}\right)=t_{A}^{f}(\lambda) e^{\Lambda^{f}\left(2 L_{A}-L\right)} E_{+}^{f}+\text { h.o.t., }
$$

where $t_{A}^{f}(\lambda)=t^{f}\left(\lambda ; L_{A}\right)+$ exponentially small terms, with $t^{f}\left(\lambda ; L_{A}\right)$ the transmission function, defined in Lemma 3.2 for the stability analysis of the fundamental periodic solution with period $2 L_{A}$. Similarly, on $\left[2 L_{A}, 2 L\right]$, the matrix $A_{p, A B}(\xi)$ is exponentially close to $A_{B}(\xi)$. Hence, the solution $\phi^{*}(\xi)$ to $(7.1)$, defined by $\phi^{*}\left(2 L_{A}\right)=e^{-\Lambda^{f} L_{B}} E_{+}^{f}$, behaves to leading order as the solution $\phi_{1}^{f}$, with $L=L B$, defined in Lemma 3.2. Hence, there exists a transmission function $t_{B}^{f}(\lambda)$, such that up to exponentially small terms,

$$
\phi^{*}(2 L)=t_{B}^{f}(\lambda) e^{\Lambda^{f} L_{B}} E_{+}^{f}+\text { h.o.t. }
$$

where $t_{B}^{f}(\lambda)=t^{f}\left(\lambda ; L_{B}\right)+$ exponentially small terms, with $t^{f}\left(\lambda ; L_{B}\right)$ the transmission function, defined in Lemma 3.2 for $L=L_{B}$. Since $\phi_{1}^{f}\left(2 L_{A}\right)$ is to leading order a multiple of $\phi^{*}\left(2 L_{A}\right)$, we have

$$
e^{-\Lambda^{f} L} \phi_{1}^{f}(2 L)=t_{A B}^{f}(\lambda) E_{+}^{f}
$$

up to exponentially small terms spanned by $\left\{E_{-}^{f}, E_{+}^{s}, E_{-}^{s}\right\}$, and

$$
t_{A B}^{f}(\lambda)=t_{A}^{f}(\lambda) t_{B}^{f}(\lambda)+\text { h.o.t. }=t^{f}\left(\lambda ; L_{A}\right) t^{f}\left(\lambda ; L_{B}\right)+\text { h.o.t. }
$$

where all higher order terms are exponentially small. It was shown in Section 3.1, that the zeroes of $t^{f}\left(\lambda ; L_{A}\right)$ and $t^{f}\left(\lambda ; L_{B}\right)$ are close to the eigenvalues $\lambda_{j}^{r}$ of the reduced eigenvalue problem. Hence, we conclude that $t_{A B}^{f}(\lambda)$ has no zeroes in $\mathbb{C}_{r}(1.8)$.

We again define $\phi_{2}^{f}$ by $\phi_{2}^{f}(\xi)=R \phi_{1}^{f}(-\xi)$.

Following Subsection 3.2, we define two slow solutions $\phi_{1,2}^{s}$ that are independent of $\phi_{1,2}^{f}$ and that span a linear subspace of solutions $\Phi^{s}(\xi)$ that is invariant under translations by $2 L$. Since the ideas in the proof of Lemma 3.6 also apply to (7.1), we know that in all outer regions such solutions are exponentially close to $\operatorname{span}\left\{E_{+}^{s}, E_{-}^{s}\right\}$. Therefore, $\Phi^{s}(0)$ is spanned by the vectors

$$
\mathbf{v}_{1}^{s}=E_{+}^{s}+\text { exp. small and } \mathbf{v}_{2}^{s}=E_{-}^{s}+\text { exp. small. }
$$

Together with the vectors

$$
\begin{aligned}
& \mathbf{v}_{1}^{f}=\phi_{1}^{f}(0)=e^{-\Lambda^{f} L} E_{+}^{f}, \\
& \mathbf{v}_{2}^{f}=\phi_{2}^{f}(0)=t_{A B}^{f}(\lambda) e^{\Lambda^{f} L} E_{-}^{f}+\text { h.o.t., }
\end{aligned}
$$

$V_{A B}=\left\{\mathbf{v}_{1}^{f}, \mathbf{v}_{2}^{f}, \mathbf{v}_{1}^{s}, \mathbf{v}_{2}^{s}\right\}$ is a basis of $\mathbb{C}^{4}$. Note that, for technical reasons, this choice of the slow base vectors differs from the choice of the slow base vectors in (3.17) by a factor $1 / \Delta$, respectively $\Delta$. With respect to $V_{A B}$, the monodromy matrix $M_{A B}$ has the form

$$
M_{A B}(\lambda)=\left(\begin{array}{cc}
M_{A B}^{f}(\lambda) & 0 \\
B(\lambda) & M_{A B}^{s}(\lambda)
\end{array}\right),
$$

as in (3.18), so that the Evans function again splits into a product of a fast and a slow Evans function,

$$
\mathcal{D}_{A B}(\lambda, \gamma)=\operatorname{det}\left[M_{A B}^{s}-\gamma I\right] \operatorname{det}\left[M_{A B}^{f}-\gamma I\right]=\mathcal{D}_{A B}^{s}(\lambda, \gamma) \mathcal{D}_{A B}^{f}(\lambda, \gamma) .
$$

The same reasoning that was applied to the eigenvalues of $M^{f}$ in Section 3.3, also applies to the eigenvalues of $M_{A B}^{f}$. Hence, $M_{A B}^{f}(\lambda)$ has no $\mathcal{O}(1)$ eigenvalues if $\lambda \in \mathbb{C}_{r}$ : there are no fast $\gamma$-eigenvalues in $\mathbb{C}_{r}$. 
The matrix $M_{A B}^{s}$ is easily determined by using the similarities between the AB-solution and the fundamental solution. Consider the slow solutions $\phi_{1,2}^{s}(\xi)$ defined by

$$
\phi_{1}^{s}(0)=e^{-\Lambda^{s} L_{A}} \mathbf{v}_{1}^{s}=\Delta_{A} \mathbf{v}_{1}^{s}, \quad \phi_{2}^{s}(0)=e^{\Lambda^{s} L_{A}} \mathbf{v}_{2}^{s}=\frac{1}{\Delta_{A}} \mathbf{v}_{2}^{s},
$$

where $\Delta_{A}=\Delta\left(\lambda, L_{A}\right)(1.11)$. In $\mathcal{I}_{0}$, these solutions are given by

$$
\begin{aligned}
& \phi_{1}^{s}(\xi)=E_{+}^{s} e^{\Lambda^{s}\left(\xi-L_{A}\right)}+\text { exp. small } \\
& \phi_{2}^{s}(\xi)=E_{-}^{s} e^{-\Lambda^{s}\left(\xi-L_{A}\right)}+\text { exp. small }
\end{aligned}
$$

As in (3.13), there exist transmission functions $t_{i j}^{A}=t_{i j}^{A}(\lambda)$, such that for $\xi \in \mathcal{I}_{1}$

$$
\begin{aligned}
& \phi_{1}^{s}(\xi)=t_{11}^{A} E_{+}^{s} e^{\Lambda^{s}\left(\xi-L_{A}\right)}+t_{21}^{A} E_{-}^{s} e^{-\Lambda^{s}\left(\xi-L_{A}\right)}+\text { exp. small } \\
& \phi_{2}^{s}(\xi)=t_{12}^{A} E_{+}^{s} e^{\Lambda^{s}\left(\xi-L_{A}\right)}+t_{22}^{A} E_{-}^{s} e^{-\Lambda^{s}\left(\xi-L_{A}\right)}+\text { exp. small, }
\end{aligned}
$$

Hence, $\xi=2 L_{A}$, we have

$$
\begin{aligned}
& \phi_{1}^{s}(\xi)=t_{11}^{A} \mathbf{v}_{1}^{s} e^{\Lambda^{s} L_{A}}+t_{21}^{A} \mathbf{v}_{2}^{s} e^{-\Lambda^{s} L_{A}}+\text { exp. small } \\
& \phi_{2}^{s}(\xi)=t_{12}^{A} \mathbf{v}_{1}^{s} e^{\Lambda^{s} L_{A}}+t_{22}^{A} \mathbf{v}_{2}^{s} e^{-\Lambda^{s} L_{A}}+\text { exp. small. }
\end{aligned}
$$

With respect to $\left\{\mathbf{v}_{1}^{s}, \mathbf{v}_{2}^{s}\right\}$, the transmission over the A-pulse is at leading order given by

$$
M_{A}^{s}=M_{A}^{s}(\lambda)=\left(\begin{array}{cc}
t_{11}^{A} \frac{1}{\Delta_{A}^{2}} & t_{12}^{A} \\
t_{21}^{A} & t_{22}^{A} \Delta_{A}^{2}
\end{array}\right) .
$$

Note that due to the different choice of basis, there appear no factors $\Delta_{A}^{2}$ and $1 / \Delta_{A}^{2}$ in the offdiagonal elements of $M_{A}^{s}$, as opposed to $M^{s}$ (3.14). Since for $\xi \in\left[0,2 L_{A}\right]$, the matrix $A_{p, A B}(\xi)$ is uniformly exponentially close to $A_{p, A}(\xi)$, the transmission functions $t_{i j}^{A}$ are to leading order the same as the transmission functions $t_{i j}(4.16)$, that were derived in the stability analysis of the fundamental periodic solution with period $2 L_{A}$.

Now, by essentially the same arguments, the transmission over the B-pulse, i.e. from $\xi=2 L_{A}$ to $\xi=2 L=2 L_{A}+2 L_{B}$, is up to exponentially small errors given by

$$
\begin{aligned}
\Delta_{B} \mathbf{v}_{1}^{s} & \longmapsto t_{11}^{B} \mathbf{v}_{1}^{s} \frac{1}{\Delta_{B}}+t_{21}^{B} \mathbf{v}_{2}^{s} \Delta_{B} \\
\frac{1}{\Delta_{B}} \mathbf{v}_{2}^{s} & \longmapsto t_{12}^{B} \mathbf{v}_{1}^{s} \frac{1}{\Delta_{B}}+t_{22}^{B} \mathbf{v}_{2}^{s} \Delta_{B}
\end{aligned}
$$

where $\Delta_{B}=\Delta\left(\lambda, L_{B}\right)$. Thus, with respect to the basis $\left\{\mathbf{v}_{1}^{s}, \mathbf{v}_{2}^{s}\right\}$, the transmission over the B-pulse is described by the matrix

$$
M_{B}^{s}=M_{B}^{s}(\lambda)=\left(\begin{array}{cc}
t_{11}^{B} \frac{1}{\Delta_{B}^{2}} & t_{12}^{B} \\
t_{21}^{B} & t_{22}^{B} \Delta_{B}^{2}
\end{array}\right) .
$$

Combining the results over the A- and B-pulses, the total transformation is given by

$$
M_{A B}^{s}(\lambda)=M_{B}^{s}(\lambda) M_{A}^{s}(\lambda),
$$

i.e. by

$$
M_{A B}^{s}(\lambda)=\left(\begin{array}{cc}
\frac{1}{\Delta_{B}^{2}}\left(1+\frac{\mathcal{J}_{B}}{2 \Lambda^{s}}\right) & \frac{\mathcal{J}_{B}}{2 \Lambda^{s}} \\
-\frac{\mathcal{J}_{B}}{2 \Lambda^{s}} & \Delta_{B}^{2}\left(1-\frac{\mathcal{J}_{B}}{2 \Lambda^{s}}\right)
\end{array}\right)\left(\begin{array}{cc}
\frac{1}{\Delta_{A}^{2}}\left(1+\frac{\mathcal{J}_{A}}{2 \Lambda^{s}}\right) & \frac{\mathcal{J}_{A}}{2 \Lambda^{s}} \\
-\frac{\mathcal{J}_{A}}{2 \Lambda^{s}} & \Delta_{A}^{2}\left(1-\frac{\mathcal{J}_{A}}{2 \Lambda^{s}}\right)
\end{array}\right)
$$

where $\frac{\mathcal{J}_{A, B}}{2 \Lambda^{s}}=\frac{\mathcal{J}_{A, B}(\lambda, \varepsilon)}{2 \Lambda^{s}(\lambda, \varepsilon)}=\mathcal{O}(1)$ is given in (4.17) with $L=L_{A, B}$. The above analysis yields the following counterpart of Proposition 3.8 . 
Proposition 7.2 For all $\lambda \in \mathbb{C}_{r}$ and $\gamma \in \mathbf{S}^{1}$, the Evans function $\mathcal{D}_{A B}(\lambda, \gamma)$ can be decomposed into a product of a fast and a slow component. The fast Evans function $\mathcal{D}_{A B}^{f}(\lambda, \gamma)$ is non-zero in $\mathbb{C}_{r}$, all $\gamma$-eigenvalues $\lambda=\lambda(\gamma, \varepsilon) \in \mathbb{C}_{r}$ of (7.1) must be zeroes of the slow Evans function $\mathcal{D}_{A B}^{s}(\lambda, \gamma) ; \lambda=\lambda(\gamma, \varepsilon)=\lambda(\gamma, 0)+\mathcal{O}(\varepsilon)$ is a $\gamma$-eigenvalue if $\lambda(\gamma, 0)$ solves

$\operatorname{Tr}\left(M_{A B}^{s}(\lambda)\right)=\frac{1}{\Delta^{2}}\left(1+\frac{\mathcal{J}_{A}}{2 \Lambda^{s}}\right)\left(1+\frac{\mathcal{J}_{B}}{2 \Lambda^{s}}\right)+\Delta^{2}\left(1-\frac{\mathcal{J}_{A}}{2 \Lambda^{s}}\right)\left(1-\frac{\mathcal{J}_{B}}{2 \Lambda^{s}}\right)-2 \frac{\mathcal{J}_{A}}{2 \Lambda^{s}} \frac{\mathcal{J}_{B}}{2 \Lambda^{s}} \in[-2,2]$

with $\gamma, \bar{\gamma} \in \mathbf{S}^{1}$ determined by $\operatorname{Tr}\left(M_{A B}^{s}\right)=2 \operatorname{Re}[\gamma]$, and $\Delta=\Delta_{A} \Delta_{B}=e^{-\Lambda^{s} L}$.

\subsection{Near the reduced eigenvalues}

The methods developed in Section 4.3 can directly be applied to the periodic orbits of AB-type.

Proposition 7.3 Let $0<\varepsilon \ll \delta \ll 1$ and $\mu \gg \delta,\left|\alpha_{2}\right| \gg \sqrt{\delta}$, and $\left|\alpha_{1}\right|=\mathcal{O}(1)$ with respect to $\delta$. Assume that the period $2 L_{B}$ of the B-part of periodic pattern $\left(U_{p, A B}(\xi ; L), V_{p, A B}(\xi ; L)\right)$ satisfies $\varepsilon^{2} L_{B}=\mathcal{O}(1)$ with respect to $\delta$.

(i) If $j=2 k$ is even, then eigenvalue problem (2.2) has no $\gamma$-eigenvalues in the ball $B\left(\lambda_{2 k}^{r}, \delta\right)$. (ii) If $j=2 k+1$ is odd and if $\operatorname{Tr}\left(M_{A B}^{s}\left(\lambda_{2 k+1}^{r}, 0\right)\right) \notin[-2,2]$, then for every $\gamma \in \mathbf{S}^{1}$, eigenvalue problem (7.1) has (precisely) two $\gamma$-eigenvalues $\lambda_{1,2}(\gamma) \in B\left(\lambda_{2 k+1}^{r}, \delta\right)$ (counting multiplicities); if $\operatorname{Tr}\left(M_{A B}^{s}\left(\lambda_{2 k+1}^{r}, 0\right)\right) \in[-2,2]$ then (2.2) has two $\gamma$-eigenvalues $\lambda_{1,2}(\gamma) \in B\left(\lambda_{2 k+1}^{r}, \delta\right) \cap \mathbb{R}$ for any $\gamma \in \mathbf{S}^{1}$ with $\left|\operatorname{Re}[\gamma]-\frac{1}{2} \operatorname{Tr}\left(M^{s}\left(\lambda_{2 k+1}^{r}, 0\right)\right)\right| \gg \delta$.

The main difference with Proposition 4.3 is the fact that (7.1) has two $\gamma$-eigenvalues near the odd fast reduced eigenvalues $\lambda_{2 k+1}^{r}$, which is not surprising given the observations in Remark 7.1 .

Proof. The proof is very similar to that of Proposition 4.3, therefore we focus on the differences with Proposition 4.3 and refer to the proof of that Lemma for more technical details.

(i) It follows from Proposition 7.2 and (7.2) in combination with Lemma 3.5 that the winding number $\mathcal{W}\left(\mathcal{D}_{A B}, \partial B\left(\lambda_{2 k}^{r}, \delta\right)\right)$ can be decomposed into

$$
\mathcal{W}\left(\mathcal{D}_{A B}, \partial B\right)=\mathcal{W}\left(\left(t^{r}\right)^{2}, \partial B\right)+\mathcal{W}\left(\gamma^{2}-\gamma \operatorname{Tr}\left(M_{A B}^{s}(\lambda, 0)\right)+1, \partial B\right),
$$

where we have used that $\operatorname{det}\left(M_{A B}^{s}\right)=\operatorname{det}\left(M_{B}^{s}\right) \operatorname{det}\left(M_{A}^{s}\right)=1$. The trace of $M_{A B}^{s}$ consists of a sum of products of the meromorphic expressions $\mathcal{J}_{A}(\lambda)$ and $\mathcal{J}_{B}(\lambda)$ ((7.6), Lemma 4.1). Hence, for $\delta$ small enough

$$
\mathcal{W}\left(\mathcal{D}_{A B}, \partial B\right)=\mathcal{W}\left(\left(t^{r}\right)^{2}, \partial B\right)+\mathcal{W}\left(\mathcal{R}^{2}, \partial B\right)=2-2=0
$$

for all $\gamma \in \mathbf{S}^{1}$.

(ii) If $\operatorname{Tr}\left(M_{A B}^{s}\left(\lambda_{2 k+1}^{r}, 0\right)\right) \notin[-2,2]$ then $\gamma^{2}-\gamma \operatorname{Tr}\left(M_{A B}^{s}(\lambda, 0)\right)+1 \neq 0$ for $\lambda \in B\left(\lambda_{2 k+1}^{r}, \delta\right)$, so that (7.7) reduces to $\mathcal{W}\left(\mathcal{D}_{\mathcal{A B}}, \partial B\right)=2+0=2$ for all $\gamma \in \mathbf{S}^{1}$. If $\operatorname{Tr}\left(M_{A B}^{s}\left(\lambda_{2 k+1}^{r}, 0\right)\right)=\gamma^{*} \in[-2,2]$, then this argument can be applied for $\gamma \in \mathbf{S}^{1}$ such that $\left|\operatorname{Re}[\gamma]-\frac{1}{2} \operatorname{Tr}\left(M^{s}\left(\lambda_{2 k+1}^{r}, 0\right)\right)\right| \gg \delta$.

In the next section we will find that the periodic patterns of AB-type are always unstable with respect to the $\mathcal{O}(1)$ eigenvalues. Hence, the small (and again $\mathcal{O}\left(\varepsilon^{4}\right)$ ) $\gamma$-eigenvalues are less relevant for the stability of the AB-patterns (on $\mathbb{R}$, see Section 8 ). Nevertheless, we will very briefly sketch a procedure - that is very similar to that of Section 5 (but computationally more involved) - by which the small eigenvalues can be determined explicitly.

We write the eigenfunctions as a perturbation of the derivative of the A-part $\left(\xi \in\left[0,2 L_{A}\right]\right)$ and 
of the B-part $\left(\xi \in\left[2 L_{A}, 2 L\right]\right)$ of the pattern, i.e. we make the ansatz

$$
\begin{array}{ll}
\phi_{\gamma}(\xi)=\phi_{0}(\xi)+\varepsilon^{2} \phi_{1}^{A}(\xi) & \text { for } \xi \in\left[0,2 L_{A}\right] \\
\phi_{\gamma}(\xi)=\theta \phi_{0}(\xi)+\varepsilon^{2} \phi_{1}^{B}(\xi) & \text { for } \xi \in\left[2 L_{A}, 2 L\right],
\end{array}
$$

where $\phi_{0}(\xi)=\left(\dot{U}_{p, A B}(\xi), \ddot{U}_{p, A B}(\xi), \dot{V}_{p, A B}(\xi), \ddot{V}_{p, A B}(\xi)\right)^{t}$ and $\theta$ is a constant (not necessarily $\in \mathbf{S}^{1}$ ), that needs to be determined in the process. By the methods of Section 5 , we find at leading order over the A-region $\left[0,2 L_{A}\right]$ that

$$
\lambda_{A}(\gamma)=\varepsilon^{2} \frac{\left|\alpha_{2}\right|}{\beta_{2}+1} \frac{1}{U_{0}\left(L_{A}\right)} \dot{u}_{1}^{A}(0 ; \gamma) \frac{\int w_{h}^{\beta_{2}+1} d \xi}{\int \dot{w}_{h}^{2} d \xi}
$$

and over the B-region $\left[2 L_{A}, 2 L\right]$ that

$$
\lambda_{B}(\gamma)=\varepsilon^{2} \frac{\left|\alpha_{2}\right|}{\beta_{2}+1} \frac{1}{U_{0}\left(L_{B}\right)} \frac{1}{\theta} \dot{u}_{1}^{B}(0 ; \gamma) \frac{\int w_{h}^{\beta_{2}+1} d \xi}{\int \dot{w}_{h}^{2} d \xi},
$$

As in Section 5 we introduce $2 \times 2$ constants $c_{1,2}^{A, B}$ that describe the decomposition of $\phi_{1}^{A, B}(\xi)$ with respect to slow solutions $\left\{\phi_{1}^{s}\left(\xi ; L_{A, B}\right), \phi_{1}^{s}\left(\xi ; L_{A, B}\right)\right\}$. Thus, we have introduced in total 5 unknown constants $\left(\theta, c_{1,2}^{A, B}\right)$. The $\mathcal{O}\left(\varepsilon^{4}\right) \gamma$-eigenvalues are now determined by imposing 5 conditions: $2 \gamma$-boundary conditions, 2 matching conditions in $\xi=2 L_{A}$, and $\lambda_{A}(\gamma)=\lambda_{B}(\gamma)$.

Remark 7.4 As an example of a more complex pattern, we may consider the AABB-pattern $\left(U_{p, A A B B}(\xi), V_{p, A A B B}(\xi)\right)$ that consists as solution of $(1.2)$ on $\mathbb{R}$ of a periodic array of two A-pulses at distance $2 L_{A}$, followed by two B-pulses at distance $2 L_{B}[6]$. It can be shown by an approach that is completely analogous to that of Section 7.1 that the part of the spectrum of the stability problem associated to $\left(U_{p, A A B B}(\xi), V_{p, A A B B}(\xi)\right)$ in $\mathbb{C}_{r}$ is determined by the matrix $M_{A A B B}^{s}(\lambda)$, with

$$
M_{A A B B}^{s}(\lambda)=M_{B}^{s}(\lambda) M_{B}^{s}(\lambda) M_{A}^{s}(\lambda) M_{A}^{s}(\lambda) .
$$

Hence, the equivalent of Proposition 7.2 can be formulated immediately, with (7.6) replaced by the condition $\operatorname{Tr}\left(M_{A A B B}^{s}(\lambda)\right) \in[-2,2]$. This expression is a polynomial of degree 4 in the meromorphic functions $\mathcal{J}_{A}(\lambda)$ and $\mathcal{J}_{B}(\lambda)$, so that we obtain the equivalent of Proposition 7.3 in which there are $4 \gamma$-eigenvalues near the odd fast reduced eigenvalues $\lambda_{2 k+1}^{r}$ for every allowed $\gamma \in \mathbf{S}^{1}$. An analysis near the saddle-node/period-doubling bifurcation like that of Lemma 7.5 below will show that there again are $\mathcal{O}(1)$ unstable eigenvalues, i.e. that the AABB-pattern $\left(U_{p, A A B B}(\xi), V_{p, A A B B}(\xi)\right)$ is unstable.

\subsection{Stability analysis of periodic orbits of AB-type}

As for the case of fundamental patterns, expression (7.6) can be explicitly evaluated with the aid of Mathematica. The results of such an evaluation for the classical Gierer-Meinhardt case (1.4) are shown in Figure 8. We see in this Figure that for $L>2 L_{S N}$, the AB periodic solution $\left(U_{p, A B}(\xi ; L), V_{p, A B}(\xi ; L)\right.$ is unstable with respect to real $\mathcal{O}(1) \gamma$-eigenvalues (in the classical case). This observation can be proven for general parameter combinations.

Lemma 7.5 There exists a critical length $L=L^{*}(\mu)>2 L_{S N}(\mu)$ such that the stability problem associated to the solution $\left(U_{p, A B}(\xi ; L), V_{p, A B}(\xi ; L)\right)$ of (1.2) of AB-type, has positive $\mathcal{O}(1) \gamma$ eigenvalues for all $L \in\left(L_{S N}(\mu), L^{*}(\mu)\right)$.

Similar to the case of the destabilization of the fundamental patterns of type A (Corollary 6.6), there seems to be a contradiction with the results of [28] in which patterns of AB-type are studied on a bounded domain. However, this is again not the case - see Section 8.2. 

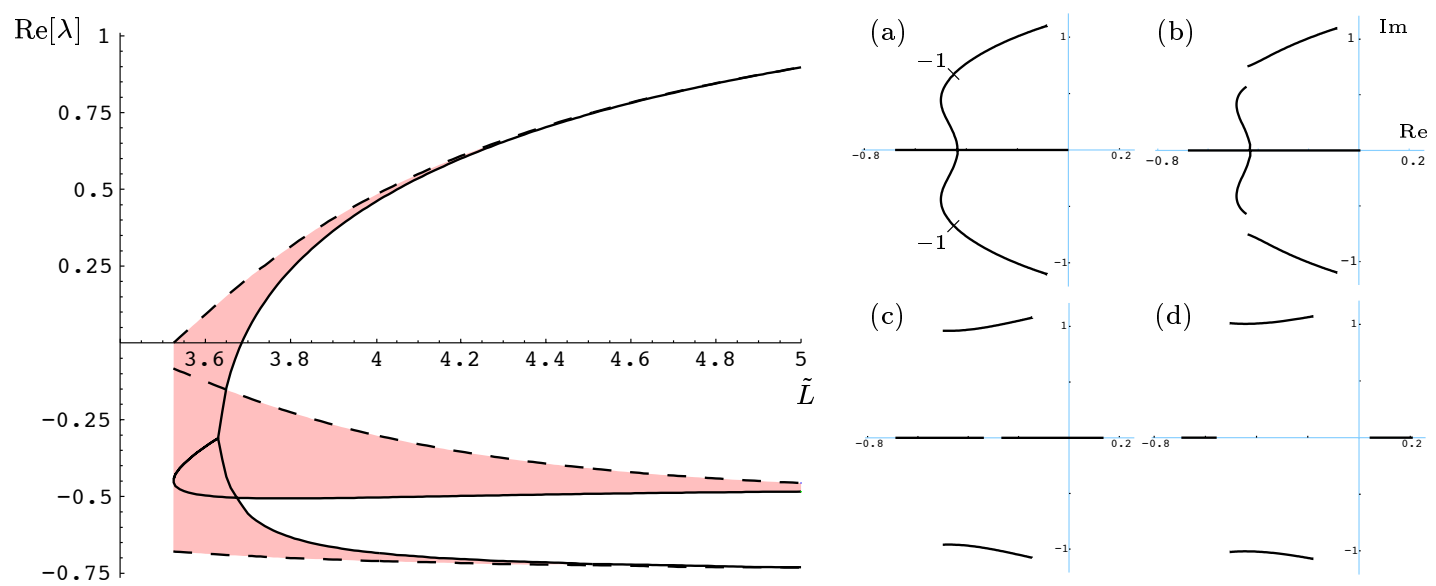

Figure 8: The real part of (all possible) $\mathcal{O}(1) \gamma$-eigenfunctions as function of $\tilde{L}=\varepsilon^{2} L$ for the periodic solution $\left(U_{p, A B}(\xi ; L), V_{p, A B}(\xi ; L)\right.$ of AB-type of the classical Gierer-Meinhardt equation (1.2) with (1.4) with $\mu=1.0$ and $\varepsilon=0.1$. Note that $\tilde{L} \geq 2 \tilde{L}_{\mathrm{SN}}=|\log [\sqrt{2}-1]|=$ $1.762 \ldots$ The dashed line denotes (the real part of) the 1-eigenvalues, the solid line the -1 eigenvalues; all other $\mathcal{O}(1) \gamma$-eigenvalues are in the grey area. Subfigures (a)-(d) show the curves of $\gamma$-eigenvalues for $\tilde{L}=2 \tilde{L}_{\mathrm{SN}}, 1.765,1.82,1.85$; (a) also appears in Figure $3(\mathrm{a})$, with a different $\gamma$-parameterization, the saddle-node/period-doubling break-up point at $\gamma=-1$ in (a) corresponds to $\gamma= \pm i$ in 3(a) (Remark 7.1). Note also that as $L, \tilde{L} \rightarrow \infty$, the 4 curves of $\gamma$-eigenvalues approach the eigenvalues $\lambda_{h}, \tilde{\lambda_{h}}$ of the homoclinic limit [3], and $\lambda_{0}^{r}=\frac{5}{4}, \lambda_{2}^{r}=\frac{3}{4}-$ as described in Lemma 7.7.

Proof. The proof is based on a local analysis of $\operatorname{Tr}\left(M_{A B}^{s}(0,0)\right)$, i.e. (7.6) at $\lambda=0$, near the saddle-node bifurcation, i.e. for both $L_{A}$ and $L_{B}$ close to $L_{S N}$. Thus, we introduce the artificial small parameter $\delta$ with $0<\varepsilon \ll \delta \ll 1$ (that is not necessarily related to $\delta$ in (1.7), (1.8)) and set $\nu=\nu_{S N}-\delta$ (recall that $\nu=\nu_{S N}$ determines the saddle-node bifurcation (1.24)).

It follows by $(3.2),(4.15)$ that,

$$
\frac{\mathcal{J}_{A, B}(0)}{2 \Lambda^{s}(0)}=-\left(1+\frac{1}{b}\right) \tanh \left(\varepsilon^{2} \sqrt{\mu} L_{A, B}\right)=-b \frac{1-\Delta_{A, B}^{2}}{1+\Delta_{A, B}^{2}},
$$

where we have introduced

$$
b=\frac{\beta_{2}-1}{D}>0
$$

(1.3). It follows from a straightforward perturbation analysis on $L_{A, B}=L_{A, B}\left(\nu_{S N}-\delta\right)$ that

$$
\Delta_{A}^{2}=\Delta_{S N}^{2}-d_{1} \sqrt{\delta}+d_{2} \delta+\mathcal{O}(\delta \sqrt{\delta}), \quad \Delta_{B}^{2}=\Delta_{S N}^{2}+d_{1} \sqrt{\delta}+d_{2} \delta+\mathcal{O}(\delta \sqrt{\delta})
$$

where

$$
d_{1}=\frac{1}{2} \sqrt{2} \Delta_{S N} \frac{1+\Delta_{S N}^{2}}{\sqrt{\mu} U_{S N}}, d_{2}=\frac{1}{6} \frac{1+\Delta_{S N}^{2}}{1-\Delta_{S N}^{2}} \frac{1-2 \Delta_{S N}^{4}}{\mu U_{S N}^{2}},
$$

and, by (1.27), (1.24), (1.23), (7.8),

$$
\Delta_{S N}=\Delta\left(0, L_{S N}\right)=\sqrt{b+1}-\sqrt{b}, \quad U_{S N}=U_{0}\left(L_{S N}\right)=\left[\frac{2 \sqrt{b} \sqrt{\mu}}{W\left(\beta_{1}, \beta_{2}\right) \sqrt{b+1}}\right]^{b} .
$$

Substituting all this into (7.6) at $\lambda=0$ yields by extensive, but straightforward, calculations

$$
\operatorname{Tr}\left[M_{A B}^{s}\left(0 ; L\left(\nu_{S N}-\delta\right)\right)\right]=2-\frac{16}{3} \frac{(1+b)(1+2 b)}{b} \frac{1}{\mu U_{S N}^{2}} \delta+\mathcal{O}(\delta \sqrt{\delta}),
$$


since $\operatorname{Tr}\left[M_{A B}^{s}\left(0 ; L_{S N}=L\left(\nu_{S N}\right)\right)\right]=2 \operatorname{Re}[\gamma]=2\left(\right.$ Remark 7.1). Thus, $\operatorname{Tr}\left[M_{A B}^{s}\left(0 ; L\left(\nu_{S N}-\delta\right)\right)\right] \in$ $[-2,2]$ at $\nu=\nu_{S N}-\delta$ for all allowed parameter combinations, so that $\lambda=0$ is a $\gamma$-eigenvalue of (7.1) for $\gamma \in \mathbf{S}^{1}$ with

$$
\arg (\gamma)=\frac{4 \sqrt{\delta}}{\sqrt{\mu} U_{S N}} \sqrt{\frac{(1+b)(1+2 b)}{3 b}}+\mathcal{O}(\delta)
$$

(Proposition 7.2). The existence of $\mathcal{O}(1) \gamma$-eigenvalues $\gamma(\lambda)>0$ for $L$ asymptotically close to $L_{S N}$ now follows from the continuity of $\operatorname{Tr}\left[M_{A B}^{s}(\lambda ; L)\right]$ as function of $\lambda$.

In fact, we have proved that $\lambda=0$ is a $\gamma$-eigenvalue for all $L \in\left(2 L_{\mathrm{SN}}(\mu), L_{0}^{*}(\mu)\right)$ for a certain $L_{0}^{*}(\mu)>2 L_{\mathrm{SN}}(\mu)$. For the classical Gierer-Meinhardt case with $\mu=1$ it follows that $L_{0}^{*}(1.0)=1.84 \ldots$ (see Figure $8(\mathrm{c})$ and $\left.(\mathrm{d})\right)$. Figure 8 also shows that $L^{*}(1.0)=\infty$ in the classical Gierer-Meinhardt case, with $L^{*}(\mu)$ as defined in Lemma 7.5.

Lemma 7.5 thus implies that the small eigenvalues do not play a role for the stability of $\left(U_{p, A B}(\xi ; L), V_{p, A B}(\xi ; L)\right)$ as solution on $\mathbb{R}$ if $L$ is increased from $L_{S N}(\mu)$ - a situation that is similar to that of the periodic solutions of B-type (Corollary 6.6).

Corollary 7.6 The period-doubling bifurcation of the periodic pattern $\left(U_{p, A B}(\xi), V_{p, A B}(\xi)\right)$ of AB-type from the fundamental patterns $\left(U_{p}\left(\xi ; L_{A, B}\right), V_{p}\left(\xi ; L_{A, B}\right)\right)$ generates $\mathcal{O}(1)$ unstable $\gamma$ eigenvalues.

It is a priori not excluded by Lemma 7.5 that the AB-patterns gain stability at $L=L^{*}(\mu)$. This is not the case, however. In principle, one could analyze relation (7.6) to confirm that also for general parameter combinations $L_{0}^{*}(\mu)<\infty$ and $L^{*}(\mu)=\infty$; moreover, $\lambda=0$ is a -1-eigenvalue for the AB-pattern at $L=L_{0}^{*}(\mu)$, and $\lambda=0$ cannot be a $\gamma$-eigenvalue for $L>L_{0}^{*}(\mu)$. For the parameter combination of the classical Gierer-Meinhardt problem (1.4), these statements follow from the direct evaluation of (7.6) presented in Figure 8. Here, we refrain from performing these (fairly extensive) computations and only consider the limit case $L \gg 1$, or equivalently $0<\nu \ll 1$.

This limit is especially interesting, since it is a result for periodic orbits with arbitrarily large period, but the results of [11] and [27] cannot be applied. Although the amplitude of the $B$ pulse decreases to 0 as $L \rightarrow \infty$, the $\left(U_{p, A B}(\xi), V_{p, A B}(\xi)\right)$ pattern is not exponentially close (in $L)$ over the interval $[0,2 L]$ to the localized homoclinic limit pattern (i.e. the limit of the A-pulse as $L \rightarrow \infty)$. Thus, we cannot use [11] to conclude that (7.1) only has eigenvalues near those of the homoclinic limit. In fact, this is not even correct.

Lemma 7.7 Let $0<\nu \ll 1$ and assume that condition $(A)-$ Section 6.1 - holds. Let $\left(U_{p, A B}(\xi ; L(\nu)), V_{p, A B}(\xi ; L(\nu))\right)$ be a periodic pattern of $A B$-type and let $\left(U_{h}(\xi), V_{h}(\xi)\right)$ be the homoclinic limit that appears from the A-pulse of the AB-pattern on the interval $\left[0,2 L_{A}(\nu)\right]$ in the limit $\nu \rightarrow 0$ (Theorem 1.7). Let $\lambda_{h}$ be an eigenvalue of the linearization around $\left(U_{h}(\xi), V_{h}(\xi)\right)$. For any $\gamma \in \mathbf{S}^{1}$ and any homoclinic eigenvalue $\lambda_{h}$ there exists a $\gamma$-eigenvalue $\lambda(\gamma)$ of (7.1) such that $\left|\lambda_{h}-\lambda(\gamma)\right|=\mathcal{O}(\nu, \varepsilon)$. Moreover, for any $\gamma \in \mathbf{S}^{1}$ and any even eigenvalue $\lambda_{2 k}^{r} \in \mathbb{C}_{e}, 2 k \in\{0,1, \ldots, J-1\}$ (Lemma 3.3), there exists a $\gamma$-eigenvalue $\lambda(\gamma) \in \mathbb{R}$ of (7.1) such that $\left|\lambda_{2 k}^{r}-\lambda(\gamma)\right|=\mathcal{O}\left(\nu^{D /\left(2\left(\beta_{2}-1\right)\right)}, \varepsilon\right)$.

Thus, although the B-part of the AB-pulse shrinks to 0 in the limit $\nu \rightarrow 0$, it does have a leading order effect on the spectrum of $\left(U_{p, A B}(\xi ; L(\nu)), V_{p, A B}(\xi ; L(\nu))\right)$. Unlike the homoclinic limit, there always is (real) unstable spectrum in $(7.1)-$ recall that $\lambda_{2 k}^{r}>0$. Note that the Lemma is confirmed by Figure 8, since for $\tilde{L} \gg 1$ there is real spectrum near the two fast reduced eigenvalues of the classical Gierer-Meinhardt case (1.4), $\lambda_{0}^{r}=\frac{5}{4}$ and $\lambda_{2}^{r}=-\frac{3}{4}$.

Proof. The proof follows the same approach as the proof of Lemma 6.1, with (3.20)/(1.10) replaced by (7.6), therefore, we need assumption (A) to ascertain that $\lambda_{h}$ is simple, isolated 
and $\in \mathbb{C}_{r}-$ as in Lemma 6.1. Here, we choose $\delta-(1.8)-$ such that $0<\nu \ll \delta \ll 1$.

We need to be careful about the magnitudes with respect to the small parameter $\nu$ of the two asymptotically small expressions $\varepsilon^{2} \sqrt{\mu} L_{B}(\nu)$ and $\Delta_{A}(\nu)$. Note that $\Delta_{B}=1$ at leading order, so that $\Delta=\Delta_{A} \Delta B=\Delta_{A}$ at leading order in $\nu$. A straightforward asymptotic calculation with (1.15), (1.16) and (1.21) yields that

$$
\varepsilon^{2} \sqrt{\mu} L_{B}(\nu)=\mathcal{O}\left(\nu^{\frac{1}{2 b}}\right), \quad \Delta_{A}(\nu)=\mathcal{O}(\sqrt{\nu}),
$$

with $b$ as in (7.8). If $\lambda \in \mathbb{C}_{r}$, both $\mathcal{J}_{A, B}(\lambda)$ are bounded and (7.6) reduces to

$$
t_{11}^{A}(\lambda) t_{11}^{B}(\lambda)=\left(1+\frac{\mathcal{J}_{A}}{2 \Lambda^{s}}\right)\left(1+\frac{\mathcal{J}_{B}}{2 \Lambda^{s}}\right)=\mathcal{O}\left(|\Delta|^{2}, \varepsilon\right)=\mathcal{O}(\nu, \varepsilon)
$$

(7.3), (7.4), (7.5), (7.9). Since $L_{A} \gg 1$ it follows that $t_{11}^{A}(\lambda)=t_{11}^{h}(\lambda)$ at leading order (see also (6.3)). Moreover, by (4.17) and (7.9),

$$
\left.t_{11}^{A}(\lambda) t_{11}^{B}(\lambda)=t^{f}(\lambda)(1+\mathcal{O}(\nu, \varepsilon))[1-\mathcal{R}(\lambda)) \mathcal{O}\left(\nu^{\frac{1}{2 b}}, \varepsilon\right)\right]=t^{f}(\lambda)\left(1+\mathcal{O}\left(\nu, \nu^{\frac{1}{2 b}}, \varepsilon\right)\right),
$$

so that it indeed follows by (7.10) that $\operatorname{Tr}\left[M_{A B}^{s}(\lambda ; L(\nu)] \in[-2,2]\right.$ for $\lambda(\gamma) \mathcal{O}(\nu, \varepsilon)$ close to a zero $\lambda_{h}$ of $t^{f}(\lambda)$. Since $\mathcal{R}(\lambda)$ has a (simple) pole at $\lambda=\lambda_{2 k}^{r}$ ([3], Section 4.1), expansion (7.11) will also be $\mathcal{O}(\nu, \varepsilon)$ (7.10) for $\left|\lambda_{2 k}^{r}-\lambda\right|=\mathcal{O}\left(\nu^{1 / 2 b}, \varepsilon\right)$. However, $t^{f}(\lambda)$ also has a pole near $\lambda_{2 k}^{r}$, and $t^{f}(\lambda)$ also appears in the right hand side of (7.10) - it could be incorporated in the $\mathcal{O}(\nu, \varepsilon)$ terms under the condition that $\lambda \in \mathbb{C}_{r}$, i.e. that $\mathcal{R}(\lambda)$ is bounded. In other words, it is a priori not clear that the right hand side of (7.10) really is asymptotically small if $\lambda$ is close to $\lambda_{2 k}^{r}$. Nevertheless, a next order analysis shows that the fact that $t^{f}(\lambda)=\mathcal{O}\left(\nu^{-1 / 2 b}\right)$ for $\lambda$ $\mathcal{O}\left(\nu^{1 / 2 b}, \varepsilon\right)$-close to $\lambda_{2 k}^{r}$ does not have a leading order influence on (7.10), i.e. it indeed follows that $\operatorname{Tr}\left[M_{A B}^{s}(\lambda ; L(\nu)] \in[-2,2]\right.$ for $\left|\lambda_{2 k}^{r}-\lambda\right|=\mathcal{O}\left(\nu^{1 / 2 b}, \varepsilon\right)$.

\section{Pulse patterns on bounded domains}

So far, we studied the stability of periodic pulse patterns on $\mathbb{R}$. Of course, most applications and numerical simulations require the problem to be posed on a bounded domain. In this section we apply the analysis of the preceding sections and determine the spectrum, and thus the stability, of pulse patterns $\left(U_{b}(\xi), V_{b}(\xi)\right)$ on a bounded domain $[0, X]$ with homogeneous Neumann boundary conditions.

\subsection{The structure of the spectrum}

We consider a stationary singular pulse pattern $\left(U_{b}(\xi), V_{b}(\xi)\right)$, that is a solution of $(1.2)$ on $[0, X]$ with the boundary conditions $\dot{U}_{b}(0)=\dot{V}_{b}(0)=0$ and $\dot{U}_{b}(X)=\dot{V}_{b}(X)=0$. This solution can be an equidistant array of identical pulses (of A- or B-type), or a combination of A- and B-pulses. In fact, we can distinguish between three types of patterns $\left(U_{b}(\xi), V_{b}(\xi)\right)$ (see also $\left.[6]\right)$ :

(I) All localized pulses of $V_{b}(\xi)$ are in the interior of $[0, X]$, i.e. $V_{b}(\xi)$ is exponentially small at $\xi=0, X$ and $U_{b}(\xi)$ has local minima at the boundaries.

(H) $V_{b}(\xi)$ has 'half a pulse' at both boundaries, i.e. both $V_{b}(\xi)$ and $U_{b}(\xi)$ are at an $\mathcal{O}(1)$ local maximum at $\xi=0, X$.

(IH) $V_{b}(\xi)$ has half a pulse at one of the boundaries and is exponentially small at the other. Without loss of generality we may assume that the 'half-pulse' is situated at $\xi=0$.

We refer to Figure 10 for examples of patterns of type $(\mathrm{I})$ and $(\mathrm{H})$. The analysis in this section will reveal that different types may give different stability properties, even if the number and 
the type of the pulses is the same, see especially Corollary 8.3 and the simulations depicted in Figure 10.

By the homogeneous Neumann boundary conditions and the reversibility symmetry of (1.2), $\left(U_{b}(\xi), V_{b}(\xi)\right)$ can be extended to a solution of $(1.2)$ on $[-X, X]$, i.e. $\left(\hat{U}_{b}(\xi), \hat{V}_{b}(\xi)\right)=$ $R\left(U_{b}(-\xi), V_{b}(-\xi)\right)$ with $R$ as defined in (2.5). It follows from the homogeneous Neumann boundary conditions at $\xi=X$ that $\hat{U}_{b}(-X)=U_{b}(X)$ and $d / d \xi \hat{U}_{b}(-X)=d / d \xi U_{b}(X)$. Thus, there is a uniquely determined solution $\left(U_{X, p}(\xi), V_{X, p}(\xi)\right)$ of $(1.2)$ associated to $\left(U_{b}(\xi), V_{b}(\xi)\right)$ that is $2 X$-periodic on $\mathbb{R}$ and is such that $\left(U_{X, p}(\xi), V_{X, p}(\xi)\right) \equiv\left(U_{b}(\xi), V_{b}(\xi)\right)$ on $[0, X]$.

An eigenfunction $\phi_{b}(\xi)$ of the linearized eigenvalue problem associated to $\left(U_{b}(\xi), V_{b}(\xi)\right)$ can be extended in a similar fashion to a $2 X$-periodic solution $\phi_{X, p}(\xi)$ of the stability problem $(2.2)$ associated to $\left(U_{X, p}(\xi), V_{X, p}(\xi)\right)$. As the thus constructed $\phi_{X, p}(\xi)$ is a $2 X$-periodic solution of $(2.2)$, it is by definition a 1-eigenfunction - an eigenfunction associated to a 1-eigenvalue - of (2.2). Note that not all 1-eigenfunctions of (2.2) correspond to eigenfunctions associated to $\left(U_{b}(\xi), V_{b}(\xi)\right)$. For instance, the derivative of $\left(U_{X, p}(\xi), V_{X, p}(\xi)\right)$ is a 1-eigenfunction that does not satisfy the (homogeneous) Neumann boundary conditions $\left(d / d \xi U_{b}(\xi)\right.$ and $d / d \xi V_{b}(\xi)$ are odd at $\xi=0, X)$.

Most often, the minimal period $2 L$ (by definition) of $\left(U_{X, p}(\xi), V_{X, p}(\xi)\right)$ is smaller than $2 X$. We define $N$ by $N=X / L$ and $\left(U_{p}(\xi ; L), V_{p}(\xi ; L)\right)$ as the periodic solution of $(1.2)$ of minimal period $2 L$ such that $\left(U_{p}(\xi), V_{p}(\xi)\right) \equiv\left(U_{X, p}(\xi), V_{X, p}(\xi)\right)$ on a certain subinterval of $[-X, X]$ of length $2 L$. Note that $N$ cannot be even for patterns $\left(U_{b}(\xi), V_{b}(\xi)\right.$ of type $(\mathrm{IH})$, that $N$ is even for patterns of type (I) and $(\mathrm{H})$ if $\left(U_{b}(\xi), V_{b}(\xi)\right)$ consists of identical pulses, and that $N$ can be either even or odd if $\left(U_{b}(\xi), V_{b}(\xi)\right)$ is of type $(\mathrm{I}),(\mathrm{H})$ and consists different (A- and B-)pulses. If $N>1$, it is natural to express the eigenvalues of the linear stability problem associated to $\left(U_{b}(\xi), V_{b}(\xi)\right)$ in terms of the $\gamma$-eigenvalues associated to $\left(U_{p}(\xi ; L), V_{p}(\xi ; L)\right)$.

Lemma 8.1 Let $\left(U_{b}(\xi), V_{b}(\xi)\right)$ be a stationary pulse solution of (1.2) on $[0, X]$ that satisfies homogeneous boundary conditions and let $\left(U_{p}(\xi ; L), V_{p}(\xi ; L)\right)$ be the corresponding periodic solution on $\mathbb{R}$ that has minimal period $2 L=2 X / N$ for $N=1,2,3, \ldots$. Then, $\lambda \in \mathbb{C}_{e}$ can only be an eigenvalue of the stability problem associated to $\left(U_{b}(\xi), V_{b}(\xi)\right)$, if $\lambda=\lambda(\gamma)$ is a $\gamma$-eigenvalue of (2.2) such that $\gamma \in \Gamma_{N}$, where

$$
\Gamma_{N}=\left\{\gamma \in \mathbf{S}^{1} \mid \gamma^{N}=1\right\}
$$

Proof. The proof follows immediately from the arguments in Remark 2.6. See also Proposition 3.1 in [10] for a more general version of this Lemma.

At this point, it is not clear yet which of the $\gamma \in \Gamma_{N}$-eigenvalues of (2.2) may give rise to an eigenfunction that satisfies the homogeneous Neumann boundary conditions on $[0, X]$. By construction, an eigenfunction of the linear problem associated to $\left(U_{b}(\xi), V_{b}(\xi)\right)$ must be symmetric at both endpoints of the interval (see Remark 2.5). In general, a $\gamma$-eigenfunction $\phi(\xi)$ of (2.2) associated to the minimal period periodic solution $\left(U_{p}(\xi ; L), V_{p}(\xi ; L)\right)$ is not symmetric. However, we know that $\lambda$ also is a $\bar{\gamma}$-eigenvalue with $\bar{\gamma}$-eigenfunction $R \phi\left(\xi_{0}-\xi\right)$ - where $\xi_{0}$ is a point of symmetry of $\left(U_{p}(\xi ; L), V_{p}(\xi ; L)\right)$ (Section 2). If these functions are independent, these two eigenfunctions may be used to construct an eigenfunction to the problem associated to $\left(U_{b}(\xi), V_{b}(\xi)\right)$, i.e. a function that is symmetric in $\xi=0$ and $\xi=X$.

Recall that we can also use the symmetry operator $R$ to characterize whether a function $\phi(\xi)$ is symmetric in a given point $\xi_{0}, R \phi\left(\xi_{0}-\eta\right)=\theta \phi\left(\xi_{0}+\eta\right)$ for $\theta=1 ; \phi(\xi)$ is anti-symmetric in $\xi_{0}$ if $\theta=-1$ (Remark 2.5). Recall also that any $2 L$-periodic solution $\left(U_{p}(\xi ; L), V_{p}(\xi ; L)\right)$ has internal reflection points at $\xi_{n}=\xi_{0}+k L, k \in \mathbb{Z}$, where of course $\xi_{0}$ can be set to be equal to 0 ([6], Theorem 1.7). 
Proposition 8.2 Let $\left(U_{b}(\xi), V_{b}(\xi)\right)$ be a pulse solution of (1.2) on $[0, X]$ that satisfies homogeneous boundary conditions and let $\left(U_{p}(\xi ; L), V_{p}(\xi ; L)\right)$ be the corresponding periodic solution on $\mathbb{R}$ that has reflection points at $\xi_{n}=n L, n \in \mathbb{Z}$, and that has minimal period $2 L=2 X / N$ for certain $N=1,2,3, \ldots$. Then, $\lambda \in \mathbb{C}_{e}$ is an eigenvalue of the linearized equations associated to $\left(U_{b}(\xi), V_{b}(\xi)\right)$ if and only if $\lambda$ satisfies one of the following three conditions.

(i) $\lambda$ is a 1-eigenvalue of the eigenvalue problem (2.2) associated to $\left(U_{p}(\xi ; L), V_{p}(\xi ; L)\right)$ and there exists a 1-eigenfunction that is not anti-symmetric in $\xi_{n}$ for at least one $n \in \mathbb{Z}$.

(ii) $\lambda$ is a-1-eigenvalue of problem (2.2) associated to $\left(U_{p}(\xi ; L), V_{p}(\xi ; L)\right), N$ is even, and there exists an-1-eigenfunction that is not anti-symmetric in one of the endpoints of $[0, X]$.

(iii) $\lambda$ is a $\gamma$-eigenvalue of problem (2.2) associated to $\left(U_{p}(\xi ; L), V_{p}(\xi ; L)\right)$ with $\gamma \in \Gamma_{N} \backslash \mathbb{R}$.

Thus, this Proposition gives a complete characterization of the spectrum of the linearization around the (multi-)pulse pattern $\left(U_{b}(\xi), V_{b}(\xi)\right)$ on the interval $[0, X]$ (with homogeneous Neumann boundary conditions). However, it does not give information on the multiplicity of the eigenvalues. Since the multiplicity of an eigenvalue $\lambda$ is not relevant for the stability question if $\lambda \neq 0$, we do not go further into this here. Moreover, we already noticed that the 'trivial' translational eigenfunction at $\lambda=0$ does not satisfy the homogeneous Neumann boundary conditions. Hence, the presence of an eigenvalue at the origin in the stability problem associated to $\left(U_{b}(\xi), V_{b}(\xi)\right)$ immediately implies that $\left(U_{b}(\xi), V_{b}(\xi)\right)$ is about to bifurcate, i.e. that it generically is in the transition from stable to unstable (or vice versa).

Proof. Consider $\lambda_{0} \in \mathbb{C}_{e}$ such that the linearization (2.2) around $\left(U_{p}(\xi ; L), V_{p}(\xi ; L)\right)$ has a $\gamma$-eigenfunction $\phi_{p}(\xi)$. To $\phi_{p}(\xi)$, we associate its symmetrical counterpart $R \phi_{p}(-\xi)(2.5)-$ note that $R \phi_{p}(-\xi)$ is a $\bar{\gamma}$-eigenfunction of $(2.2)$. If $\phi_{p}(\xi)$ and $R \phi_{p}(-\xi)$ are independent, we can define

$$
\phi_{ \pm}(\xi)=\phi_{p}(\xi) \pm R \phi_{p}(-\xi) \text {. }
$$

By construction, $\phi_{+}(\xi)$ is symmetric in $\xi=0$, i.e. $\phi_{+}(\xi)$ satisfies the homogeneous boundary conditions at $\xi=0$, and $\phi_{-}(\xi)$ is anti-symmetric in $\xi=0$ (Remark 2.5).

Hence, we first need to determine the dimension of $\left\{\phi_{p}(\xi), R \phi_{p}(-\xi)\right\}$. Clearly, the condition $\phi_{p}(\xi)=\theta R \phi_{p}(-\xi)$ on $\mathbb{R}$ implies that $\theta= \pm 1$, i.e. $\phi_{p}(\xi)$ and $\left.R \phi_{p}(-\xi)\right\}$ can only be dependent if $\phi_{p}(\xi)$ is symmetrical or anti-symmetrical in $\xi=0$ (Remark 2.5). In that case,

$$
\phi_{p}(L+\eta)=\gamma \phi_{p}(-L+\eta)=\gamma \theta R \phi_{p}(L-\eta),
$$

for $\eta \in \mathbb{R}$, which implies that $\gamma \theta= \pm 1$. Thus, $\phi_{p}(\xi)$ and $\left.R \phi_{p}(-\xi)\right\}$ can only be dependent for $\gamma= \pm 1$. This implies that the combination $\phi_{+}(\xi)(8.2)$ is a candidate for being an eigenfunction with eigenvalue $\lambda_{0}$ of the linear problem associated to $\left(U_{b}(\xi), V_{b}(\xi)\right)$ on $[0, X]$ for $\gamma \in \Gamma_{N} \backslash \mathbb{R}$, since it is a solution of (2.2) for $\lambda=\lambda_{0}$, and it satisfies the homogeneous Neumann boundary conditions at $\xi=0$.

Before we consider the more special cases $\gamma= \pm 1$, we first prove part (iii) of the Proposition. Since $\gamma^{N}=1$, the solution $\phi_{+}(\xi)$ is symmetric in $\xi=k N L$ for $k \in \mathbb{Z}$,

$$
\begin{aligned}
\phi_{+}(k N L-\eta) & =\phi_{p}(k N L-\eta)+R \phi_{p}(-k N L+\eta) \\
& =\gamma^{k N} \phi_{p}(-k N L-\eta)+\gamma^{-k N} R \phi_{p}(k N L+\eta) \\
& =\phi_{p}(-k N L-\eta)+R \phi_{p}(k N L+\eta) \\
& =R\left[\phi_{p}(k N L+\eta)+R \phi_{p}(-k N L-\eta)\right] \\
& =R \phi_{+}(k N L+\eta)
\end{aligned}
$$

for $\eta \in \mathbb{R}$. Since $X=N L$, it follows that $\phi_{+}(\xi)$ is a solution of (2.2) that is symmetric in both $\xi=0$ and $\xi=X$, i.e. $\phi_{+}(\xi)$ is an eigenfunction at $\lambda=\lambda_{0}$ for the linear stability problem associated to $\left(U_{b}(\xi), V_{b}(\xi)\right)$. 
For the special cases $\gamma= \pm 1$, the multiplicity may be $\geq 2$, so that $\phi_{p}(\xi)$ and $\left.R \phi_{p}(-\xi)\right\}$ can be independent, and the symmetric/anti-symmetric eigenfunctions $\phi_{ \pm}(\xi)$ will exist. In such cases, $\lambda=\lambda_{0}$ is also an eigenfunction for the $\left(U_{b}(\xi), V_{b}(\xi)\right)$ stability problem. Note that these cases are covered by (i) and (ii) (where $N$ must be even to have $-1 \in \Gamma_{N}$, Lemma 8.1).

Thus, to finish the proof of (i) and (ii), we may assume that $\phi_{p}(\xi)$ is either symmetric or anti-symmetric in $\xi=0$, i.e. that $\phi_{p}(\xi)=\theta R \phi_{p}(-\xi)$ for $\theta= \pm 1$. It follows from (8.3) that $\phi_{p}(\xi)$ is also symmetric/anti-symmetric in $\xi=L$, and that the symmetry-type is the same as in $\xi=0$ for $\gamma=1$, but changes for $\gamma=-1$. More in general, for $k \in \mathbb{Z}$,

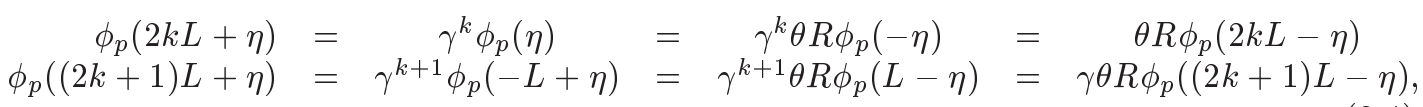

so that it follows that $\phi_{p}(\xi)$ has the same symmetry-type at every $\xi=n L$ for $\gamma=1$, and that its type alternates for $\gamma=-1$. This immediately proves part (i) of the Proposition. Since $X=N L$ and $N$ must be even in part (ii) of the Proposition (Lemma 8.1), $\phi_{p}(\xi)$ is either symmetric or anti-symmetric at both boundary points.

\section{2 $M$ identical pulses on $[0, X]$}

As an application of the results of the preceding subsection, we study the stability of a pulse pattern $\left(U_{b}(\xi), V_{b}(\xi)\right)$ that consists of $M$ identical pulses on the interval $[0, X]$. Since $\left(U_{b}(\xi), V_{b}(\xi)\right)$ may be of type (IH), we allow for $M \in \frac{1}{2} \mathbb{N} /\{0\}$. The associated $2 X$-periodic solution $\left(U_{X, p}(\xi), V_{X, p}(\xi)\right)$ consists of $2 M$ identical pulses. The periodic solution $\left(U_{p}(\xi ; L), V_{p}(\xi ; L)\right)$ of minimal period is a fundamental periodic solution (of either type A or B) with period $2 L=X / M=2 X / N$, so that $N=2 M$. By Proposition 8.2, the spectrum of the linearization around the $M$-pulse pattern $\left(U_{b}(\xi), V_{b}(\xi)\right)$ is given by the $\gamma$-eigenvalues of the eigenvalue problem $(2.2)$ associated to $\left(U_{p}(\xi ; L), V_{p}(\xi ; L)\right)$ with $\gamma \in \Gamma_{N}(8.1)$, where we need to be extra careful with the special cases $\gamma= \pm 1$ (parts (i) and (ii) of Proposition 8.2). Of course, the conclusion whether or not a \pm 1-eigenfunction of (2.2) corresponds to an eigenfunction associated to the full $\left(U_{b}(\xi), V_{b}(\xi)\right)$ pattern depends on the character of the pattern - i.e. whether it is type (I), $(\mathrm{H})$, or $(\mathrm{IH})$.

We know from the theory developed in preceding sections that the (relevant part of the) spectrum of the linearization around the fundamental periodic solution $\left(U_{p}(\xi ; L), V_{p}(\xi ; L)\right)$ consists of a curve of small $\mathcal{O}\left(\varepsilon^{4}\right) \gamma$-eigenvalues, connected to the translational eigenvalue $\lambda=0$, and a number of curves of $\mathcal{O}(1) \gamma$-eigenvalues. Recall that $\left(U_{p}(\xi ; L), V_{p}(\xi ; L)\right)$ has two reflection points per period (by construction [6], Theorem 1.7), one at $\xi=\xi_{0}=0$ (without loss of generality) such that $U_{p}(\xi ; L)$ and $V_{p}(\xi ; L)$ are at a local maximum (i.e. $\xi_{0}$ determines the center of the pulse), and one at $\xi_{1}=L$, exactly between two adjacent pulses (i.e. $U_{p}(\xi ; L)$ and $V_{p}(\xi ; L)$ are both at a local minimum).

If $\phi_{p}(\xi)$ is an eigenfunction of (2.2) that corresponds to a small $\gamma$-eigenvalue, then its $v$ component is to leading order equal to the derivative of the wave $d / d \xi V_{p}(\xi ; L)$ in a pulse region (Section 5). Since the $u$-component of $\phi_{p}(\xi)$ is at leading order 0 in a pulse region (5.1), it follows that $\phi_{p}(\xi)$ is to leading order anti-symmetric in the reflection points $\xi_{2 k}=2 k L$. By Proposition 4.3, the small $\gamma$-eigenvalues have $\gamma$-multiplicity one. Hence, $\phi_{p}(\xi)$ and $R \phi_{p}(-\xi)$ cannot be independent for $\gamma= \pm 1$, i.e. $\gamma \in \mathbf{S}^{1}$ such that $\bar{\gamma}=\gamma$, so that $\phi_{p}(\xi)$ must be exactly anti-symmetric in all reflection points $\xi_{2 k}=2 k L$ for $\gamma= \pm 1$. It follows from (8.4) that $\phi_{p}(\xi)$ is anti-symmetric in all reflection points $\xi_{n}=n L, n \in \mathbb{Z}$ for $\gamma=1$, so that $\phi_{p}(\xi)$ cannot be an eigenfunction of the stability problem associated to $\left(U_{b}(\xi), V_{b}(\xi)\right)$ for $\gamma=1$ (part (i) of Proposition 8.2). Note that we already knew this, for the small 1-eigenfunction is the derivative of the wave. On the other hand, it also follows from $(8.4)$ that $\phi_{p}(\xi)$ is symmetric at the reflection points $\xi_{2 k+1}=(2 k+1) L$ for $\gamma=-1$. Hence, the eigenfunction $\phi_{p}(\xi)$ corresponding to an $\mathcal{O}\left(\varepsilon^{4}\right)$ -1-eigenvalue of $(2.2)$ is an eigenfunction of the stability problem associated to $\left(U_{b}(\xi), V_{b}(\xi)\right)$ 
if and only if $\left(U_{b}(\xi), V_{b}(\xi)\right)$ is of type $(I)$.

The large $\gamma$-eigenfunctions are, by construction, to leading order symmetric with respect to the reflection point $\xi_{2 k}$, i.e. the centers of the pulses. This follows immediately from the fact that the solutions $w_{i n}(\xi)$ of $(4.7)$ must be even with respect to the center of the pulse - recall that the $v$-component of an $\mathcal{O}(1) \gamma$-eigenfunction $\phi_{p}(\xi)$ is to leading order a scaled version of $w_{h}(\xi)$ in the pulse region, while its $u$-component is to leading order constant (Section 4.1). Therefore, by the same arguments as applied to the small eigenvalue case, if $\lambda$ is a $\mathcal{O}(1)$ 1eigenvalue with geometric $\gamma$-multiplicity one, then the corresponding 1-eigenfunction must be exactly symmetric in all reflection points $\xi_{n}=n L$. Moreover, an $\mathcal{O}(1)-1$-eigenfunction is symmetric in the points $\xi_{2 k}$ at the centers of the pulse, and anti-symmetric at the points $\xi_{2 k+1}$ in between the pulses. Hence, the 1-eigenfunction corresponding to an $\mathcal{O}(1)$ 1-eigenvalue of (2.2) satisfies the Neumann boundary conditions at $\xi=0, X$, irrespective of the type of the $\left(U_{b}(\xi), V_{b}(\xi)\right)$ pattern, whereas the $\mathcal{O}(1)$-1-eigenfunctions satisfy the boundary conditions if and only if $\left(U_{b}(\xi), V_{b}(\xi)\right)$ is of type $(H)$.

Corollary 8.3 Let $\left(U_{b}(\xi), V_{b}(\xi)\right)$ be a pulse solution of (1.2) on $[0, X]$ that consists of $M \in$ $\frac{1}{2} \mathbb{N} /\{0\}$ identical pulses and that satisfies homogeneous Neumann boundary conditions, and let $\left(U_{p}(\xi ; L), V_{p}(\xi ; L)\right)$ be the corresponding fundamental periodic solution on $\mathbb{R}$ with minimal period $2 L=X / M=2 X / N$. Then, $\lambda \in \mathbb{C}_{e}$ is an eigenvalue of the linearized equations associated to $\left(U_{b}(\xi), V_{b}(\xi)\right)$ depending on the type of $\left(U_{b}(\xi), V_{b}(\xi)\right)$ and on the magnitude of the $\gamma$-eigenvalues of (2.2) associated to $\left(U_{p}(\xi ; L), V_{p}(\xi ; L)\right)$ :

(I) $\lambda \in \mathbb{C}_{e}$ is an eigenvalue if $\lambda$ is an $\mathcal{O}(1) \gamma$-eigenvalue with $\gamma \in \Gamma_{N} /\{-1\}$ or an $\mathcal{O}\left(\varepsilon^{4}\right)$ $\gamma$-eigenvalue with $\gamma \in \Gamma_{N} /\{1\}$.

(H) $\lambda \in \mathbb{C}_{e}$ is an eigenvalue if $\lambda$ is an $\mathcal{O}(1) \gamma$-eigenvalue with $\gamma \in \Gamma_{N}$ or an $\mathcal{O}\left(\varepsilon^{4}\right) \gamma$-eigenvalue with $\gamma \in \Gamma_{N} /\{-1,1\}$.

(IH) $\lambda \in \mathbb{C}_{e}$ is an eigenvalue if $\lambda$ is an $\mathcal{O}(1) \gamma$-eigenvalue with $\gamma \in \Gamma_{N} /\{-1\}$ or an $\mathcal{O}\left(\varepsilon^{4}\right)$ $\gamma$-eigenvalue with $\gamma \in \Gamma_{N} /\{-1,1\}$.

Note that $\Gamma_{N} \backslash \mathbb{R}=\Gamma_{2 M} \backslash \mathbb{R}=\emptyset$ for $M=\frac{1}{2}, 1$, so that $\gamma= \pm 1$ are the only possibilities for patterns consisting of a half pulse (type $(\mathrm{IH})$ ), two half pulses (type $(\mathrm{H})$ ), or one full pulse (I). As a consequence, the half pulse pattern only has eigenvalues that correspond to $\mathcal{O}(1)$ 1-eigenvalues of (2.2), the two half pulses pattern only has eigenvalues that correspond to $\mathcal{O}(1)$ \pm 1 -eigenvalues, and the one pulse pattern only has eigenvalues that correspond to $\mathcal{O}(1)$ 1eigenvalues and the $\mathcal{O}\left(\varepsilon^{4}\right)$-1-eigenvalue (that is always negative, Lemmas 5.2 and 5.3).

In combination with Lemma 5.3, Corollary 8.3 can also be used to derive a somewhat surprising result on the character of the destabilization of the $M$-pulse pattern $\left(U_{b}(\xi), V_{b}(\xi)\right)$ caused by the saddle-node (or period doubling) bifurcation, i.e. the parameter value at which the associated periodic orbits $\left(U_{p}(\xi ; L), V_{p}(\xi ; L)\right)$ changes of type A into type B. In Section 6.2, the destabilization of the fundamental pattern $\left(U_{p}(\xi ; L), V_{p}(\xi ; L)\right)$ has been studied as function of $L$. Here, it is more natural to fix $L$, i.e. to fix $X$ and $M$, and to vary the main bifurcation parameter $\mu$ of (1.2). It follows from (1.27) that for given $L$ fixed there is a critical value of $\mu, \mu_{\mathrm{SN}}(L)$ at which the pattern $\left(U_{p}(\xi ; L), V_{p}(\xi ; L)\right)$ passes through the saddle-node bifurcation. Clearly, $\left(U_{p}(\xi ; L), V_{p}(\xi ; L)\right)$ is of A-type for $\mu>\mu_{\mathrm{SN}}(L)$ and of B-type for $\mu<\mu_{\mathrm{SN}}(L)$.

We assume that the solution $\left(U_{p}(\xi ; L), V_{p}(\xi ; L)\right)$ is stable as solution of $(1.2)$ on $\mathbb{R}$ for $\mu>$ $\mu_{\mathrm{SN}}(L)$, i.e. that the A-pattern did not destabilize by a Hopf bifurcation. We know by Corollary 6.6 that the stability problem associated to $\left(U_{p}(\xi ; L), V_{p}(\xi ; L)\right)$ has $\mathcal{O}(1)$ unstable eigenvalues as soon as $\mu<\mu_{\mathrm{SN}}(L)$. However, the most unstable eigenvalue is a -1-eigenvalue (Lemma 6.4). This $\mathcal{O}(1)$-1-eigenvalue only corresponds to an eigenvalue associated to $\left(U_{b}(\xi), V_{b}(\xi)\right)$ if this pattern is of type $(\mathrm{H})$. Thus, if $\left(U_{b}(\xi), V_{b}(\xi)\right)$ is of type $(\mathrm{I})$ or $(\mathrm{IH})$, it will not be destabilized by the $\mathcal{O}(1)$-1-eigenvalue! Nevertheless, in these cases $\left(U_{b}(\xi), V_{b}(\xi)\right)$ is also destabilized at a bifurcation value that is at leading order the same as $\mu_{\mathrm{SN}}(L)$ (if $M \geq \frac{3}{2}$, see below). 

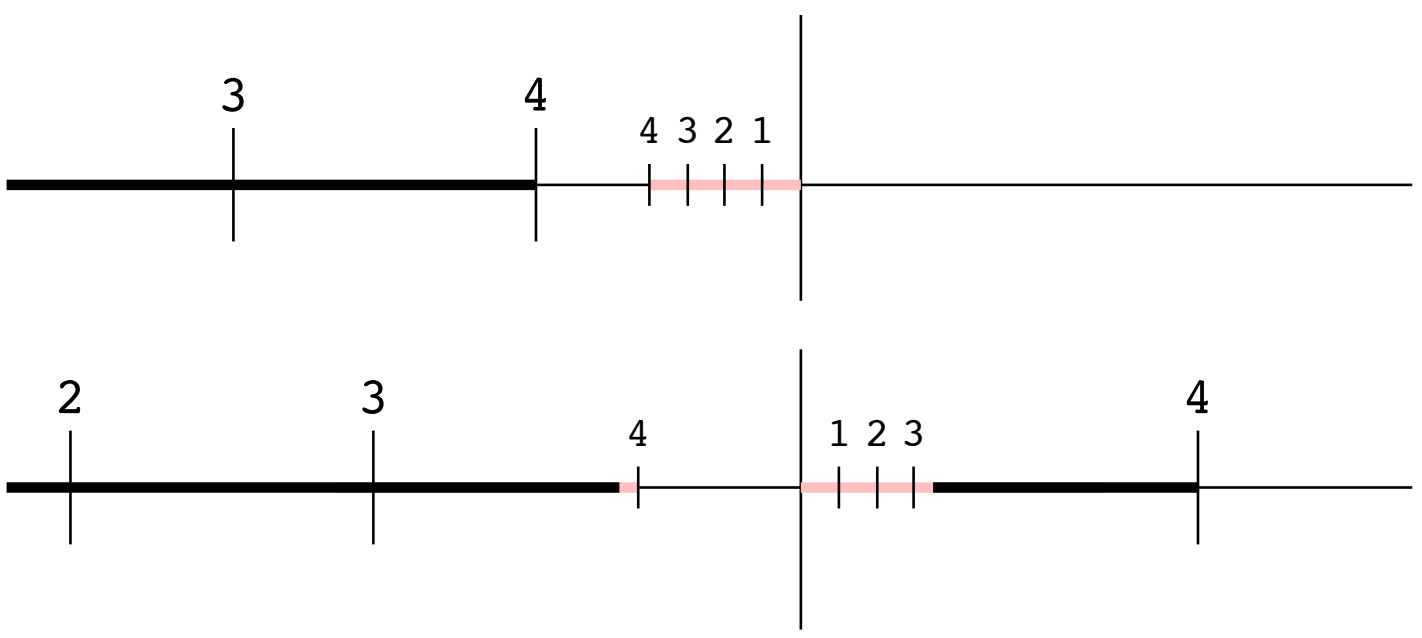

Figure 9: A sketch of the interaction of the $\mathcal{O}(1) \gamma$-eigenvalues (black) and the $\mathcal{O}\left(\varepsilon^{4}\right) \gamma$ eigenvalues (gray) as $\mu$ decreases through $\mu_{\mathrm{SN}}(L)$. The markers indicate the positions of the $\gamma$-eigenvalues with $\gamma=e^{ \pm i k \pi / 4}$ and $k=1,2,3,4\left(\lambda=0\right.$ is a small $1=e^{0}$-eigenvalue). Note that the transition between the $\mathcal{O}(1)$ and the $\mathcal{O}\left(\varepsilon^{4}\right)$ spectrum, for $\mu<\mu_{\mathrm{SN}}(L)$, has not been studied in this paper.

This can be seen by Figure 9, that gives a graphic representation of the relation between the small and the $\mathcal{O}(1)$ spectrum as $\mu$ decreases through $\mu_{\mathrm{SN}}(L)$, as determined in (the proofs of) Lemmas 5.2 and 5.3. If $\mu<\mu_{\mathrm{SN}}(L)$ with $\mu_{\mathrm{SN}}(L)-\mu=\tilde{\delta}$ for some $0<\tilde{\delta} \ll 1$, the largest $\mathcal{O}(1)$ $\gamma$-eigenvalue, $\gamma=-1$ (Lemma 6.4), is still $\gg \varepsilon^{4}$ away from 0 (for $\varepsilon$ small enough - Corollary 6.6), the entire $\mathcal{O}\left(\varepsilon^{4}\right)$ spectrum is (also) in the stable half plane (Lemma 5.2). If $\mu<\mu_{\mathrm{SN}}(L)$ with $\mu_{\mathrm{SN}}(L)-\mu=\delta \ll 1$, only a small part of the $\mathcal{O}(1)$-spectrum has passed through $\lambda=0$ (Lemma 6.4). However, except for those $\mathcal{O}(\delta)$ close to $\gamma=-1$, all (!) $\mathcal{O}\left(\varepsilon^{4}\right) \gamma$-eigenvalues have moved through the imaginary axis. This follows immediately from the structure of the graph of $\lambda$ as function of $|\arg [\gamma]|$ as described in the proof of Lemma 5.3. This means that the most unstable eigenvalue $\lambda_{b}^{*}$ of the stability problem associated to $\left(U_{b}(\xi), V_{b}(\xi)\right)$ of type (I) or (IH) on $[0, X]$ is the $\mathcal{O}\left(\varepsilon^{4}\right) \gamma$-eigenvalue with $\gamma=e^{ \pm(M-1) \pi i / M}$, i.e. the small eigenvalue with $\gamma$ as close as possible to -1 . Note that this corresponds to $k=3$ in Figure 9 . Note also that $\lambda_{b}^{*}$ does not exist if $M=\frac{1}{2}, 1$.

Corollary 8.4 Let $\left(U_{b}(\xi), V_{b}(\xi)\right)$ be a pulse solution of (1.2) on [0,X] that consists of $M \in$ $\frac{1}{2} \mathbb{N} /\{0\}$ identical pulses and that satisfies homogeneous Neumann boundary conditions, and let $\left(U_{p}(\xi ; L), V_{p}(\xi ; L)\right)$ be the corresponding fundamental periodic solution on $\mathbb{R}$ with minimal period $2 L=X / M=2 X / N$. Assume that $\left(U_{p}(\xi ; L), V_{p}(\xi ; L)\right)$ is stable as solution on $\mathbb{R}$ for $\mu>\mu_{\mathrm{SN}}(L)$.

- If $\left(U_{b}(\xi), V_{b}(\xi)\right)$ is of type $(H)$, then it is destabilized as $\mu$ decreases through $\mu_{\mathrm{SN}}(L)$ by an $\mathcal{O}(1)$ eigenvalue associated to $\gamma=-1$ in (2.2).

- If $\left(U_{b}(\xi), V_{b}(\xi)\right)$ is of type (I) or (IH) and if $M \geq \frac{3}{2}$, then it is destabilized as $\mu$ decreases through $\mu_{\mathrm{SN}}(L)$ by an $\mathcal{O}\left(\varepsilon^{4}\right)$ eigenvalue associated to $\gamma=e^{ \pm(M-1) \pi i / M}$.

- $\left(U_{b}(\xi), V_{b}(\xi)\right)$ does not destabilize as $\mu$ decreases through $\mu_{\mathrm{SN}}(L)$ if it is of type (I) or (IH) with $M=\frac{1}{2}$ or 1 .

This Corollary proves that there is no contradiction between the results presented in [17, 29] and those of the present paper. In $[17,29]$ it has been shown that the destabilization of an $M$-pulse pattern on a bounded domain at the saddle-node bifurcation is always associated with the small spectrum. However, in $[17,29]$ bounded interval patterns of type (I) are considered. In this paper, it has been shown that there are always $\mathcal{O}(1)$ unstable eigenvalues beyond the saddle- 
node bifurcation in the stability problem for (fundamental) periodic patterns on $\mathbb{R}$ (Corollary 6.6). Corollary 8.4 shows that, near the saddle-node bifurcation, the $\mathcal{O}(1)$ eigenvalues cannot be 'seen' by patterns of type (I) on a bounded domain. Hence, these patterns are destabilized by the 'less unstable' $\mathcal{O}\left(\varepsilon^{4}\right)$ eigenvalues. It is for the same reason that there is no contradiction between the statement on the instability of patterns of AB-type on $\mathbb{R}$ (Lemma 7.5) and the results obtained in [28]. In this paper, AB-patterns of type (I) (i.e. on a bounded domain) are considered, and they are shown to be unstable with respect to the $\mathcal{O}\left(\varepsilon^{4}\right)$ eigenvalues. Again, the unstable $\mathcal{O}(1)$-eigenvalues of Lemma 7.5 cannot be 'seen' by the type (I) pattern.

The statements of this Corollary are confirmed by the numerical simulations. It has been checked that the $\left(U_{b}(\xi), V_{b}(\xi)\right)$ pattern with $M=1$ indeed does not bifurcate at $\mu_{\mathrm{SN}}(L)$ if it is of (I) type, while the $M=1$ pattern of type $(\mathrm{H})$ does bifurcate at $\mu_{\mathrm{SN}}(L)$. In Figure 10 , numerical simulations of two $M=2$ patterns, of types $(\mathrm{I})$ and $(\mathrm{H})$, are presented for $\mu$ just beyond $\mu_{\mathrm{SN}}(L)$. Clearly the $(\mathrm{H})$ pattern evolves on a much faster time scale, i.e. the time scale associated to an $\mathcal{O}(1)$ unstable eigenvalue, than the (I) pattern (that has an unstable eigenvalue of $\left.\mathcal{O}\left(\varepsilon^{4}\right)\right)$. Moreover, the destabilizing eigenfunctions have been computed numerically. If $\left(U_{b}(\xi), V_{b}(\xi)\right)$ is of type (I), the (unstable) eigenfunction clearly consist (at leading order) of 'locally odd copies' of the derivative of the localized pulses (Figure 10). Hence, it is (numerically) confirmed that the destabilization is associated with the $\mathcal{O}\left(\varepsilon^{4}\right)$ eigenvalues (Section 5). The unstable eigenfunction consist of localized even pulses, associated to the solution $v_{i n}(\xi)$ of (4.6), for the (H)-pattern, i.e. the $(\mathrm{H})$-pattern is indeed destabilized by an $\mathcal{O}(1)$ eigenvalue.

Remark 8.5 Note that the first $\mathcal{O}(1)$ eigenvalue of the stability problem associated to $\left(U_{b}(\xi), V_{b}(\xi)\right)$ of type (I) or (IH) that crosses through the imaginary axis is the eigenvalue associated to $\gamma=\gamma_{M}^{*}=e^{ \pm(M-1) \pi i / M}$. By Remark 6.5 this occurs (at leading order) at

$$
\mu=\mu_{M}^{*}=\frac{M^{2}}{\left(\varepsilon^{2} X\right)^{2}}\left(\operatorname{arccosh}\left(1+\left(1+\cos \frac{\pi}{M}\right) \frac{\beta_{2}-1}{D}\right)\right)^{2},
$$

which agrees with the results found in $[17,29]$. Note that $\mu_{M}^{*}$ approaches $\mu_{\mathrm{SN}}(L)$ as $M \rightarrow \infty$ (Remark 6.5). We may also conclude that if $\mu<\mu_{\mathrm{SN}}(L)$ with $\mu_{\mathrm{SN}}(L)-\mu=\delta \ll 1$, then there is an $M_{0}(\delta)$ such that the $\mathcal{O}(1)$ eigenvalue associated to $\gamma_{M}^{*}$ is positive for $M>M_{0}(\delta)$. In other words, if one considers a pulse pattern $\left(U_{b}(\xi), V_{b}(\xi)\right)$ that consists of many identical pulses (i.e. if $M$ is large enough) as an initial condition for a numerical simulation of (1.2) with $\mu=\mu_{\mathrm{SN}}(L)-\delta$, then $\left(U_{b}(\xi), V_{b}(\xi)\right)$ will be destabilized on the fast time scale, i.e. by an $\mathcal{O}(1)$ eigenvalue, irrespective of the type of $\left(U_{b}(\xi), V_{b}(\xi)\right)$.

Remark 8.6 According to Corollary 8.3, the stability problem for a periodic pattern on a bounded domain only has finitely many eigenvalues $\lambda \in \mathbb{C}_{e}$. This implies that this stability problem must have infinitely many additional eigenvalues $\lambda \in \mathbb{C} \backslash \mathbb{C}_{e}$, i.e. in (a neighborhood of) the essential spectrum of the homoclinic limit pattern. See Remark 1.4.

Figure 10: Numerical simulations of the destabilization of two different pulse solutions $\left(U_{b}(x), V_{b}(x)\right)$ on the same bounded domain $x \in[0,12]$, both with $M=2, \mu=1.08, \varepsilon=0.3$, and (1.4). The patterns correspond to the same fundamental pattern $\left(U_{p}(\xi ; L), V_{p}(\xi ; L)\right)$ with $\tilde{L}=\varepsilon^{2} L=0.9$, but are of different types; $(I)$ in the left column, $(H)$ in the right. The bifurcation value $\mu_{\mathrm{SN}}(\tilde{L} ; \varepsilon)=(\log [\sqrt{2}-1])^{2} / \tilde{L}^{2}+\mathcal{O}(\varepsilon) \approx 0.96+\mathcal{O}(\varepsilon)>1.08$, as is found numerically. Both patterns bifurcate into the same pulse pattern of type (I) with $M=1$. In the first row, the $U$-components (thick lines) and $V$-components (thin lines) of the initial pattern are plotted. The second row shows the evolution of the amplitude of the $V$-components of the pulses; note the significant difference in the time scales. The third row presents the $V$-component of the (numerically computed) most unstable eigenfunctions. 

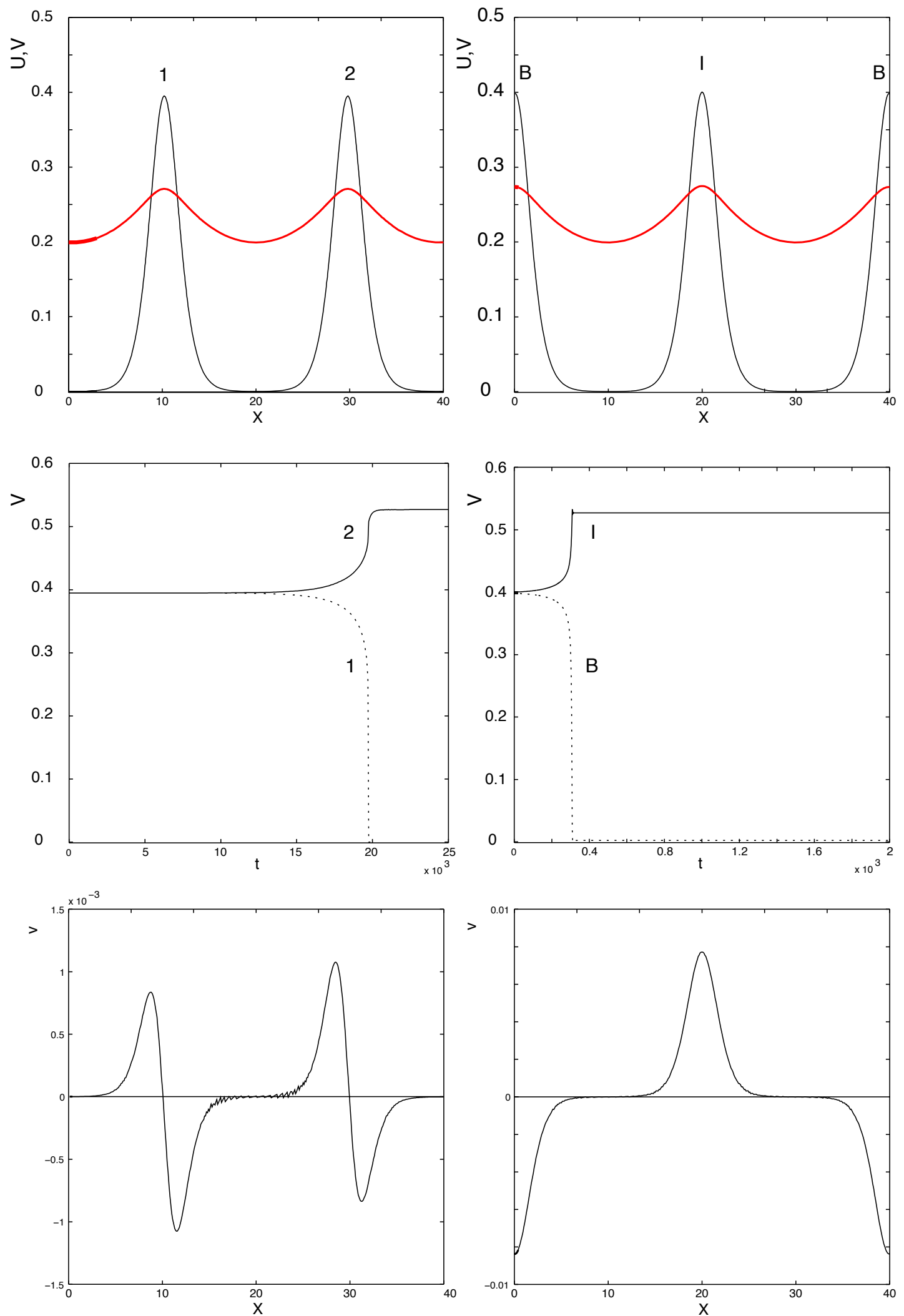

\section{References}

[1] J. Alexander, R.A. Gardner, and C.K.R.T. Jones, A topological invariant arising in the stability analysis of traveling waves, J. Reine Angew. Math. 410 (1990), 167-212. 
[2] S. Benzoni-Gavage, D. Serre, and K. Zumbrun, Alternate Evans functions and viscous shock waves, SIAM J. Math. Anal. 32 (2001), no. 5, 929-962.

[3] A. Doelman, R.A. Gardner, and T.J. Kaper, Large stable pulse solutions in reactiondiffusion equations, Indiana Univ. Math. J. 49 (2000), no. 4, 443-507.

[4] A. Doelman, R.A. Gardner, and T.J. Kaper, A stability index analysis of 1-D patterns of the Gray-Scott model, Memoirs AMS 155 (2002), no. 737.

[5] A. Doelman and T.J. Kaper, Semi-strong pulse interactions in a class of coupled reactiondiffusion equations, SIAM J. Appl. Dyn. Syst. 2 (2003), no. 1, 53-96.

[6] A. Doelman, T.J. Kaper, and H. van der Ploeg, Spatially periodic and aperiodic multi-pulse patterns in the one-dimensional Gierer-Meinhardt equation, Meth. Appl. Anal. 8 (2001), no. $2,387-414$.

[7] W. Eckhaus, Asymptotic Analysis of Singular Perturbations, North-Holland, 1979.

[8] E.G. Eszter, Evans function analysis of the stability of periodic travelling wave solutions of the FitzHugh-Nagumo system, PhD thesis, U. of Massachusetts, Amherst, 1999.

[9] N. Fenichel, Persistence and smoothness of invariant manifolds for flows, Indiana Univ. Math. J. 21 (1971), 193-226.

[10] R.A. Gardner, On the structure of the spectra of periodic travelling waves, J. Math. Pures Appl. (9) 72 (1993), no. 5, 415-439.

[11] R.A. Gardner, Spectral analysis of long wavelength periodic waves and applications, J. Reine Angew. Math. 491 (1997), 149-181.

[12] R.A. Gardner and C.K.R.T. Jones, Stability of the travelling wave solutions of diffusive predator-prey systems, Trans. AMS 327 (1991), 465-524.

[13] R.A. Gardner and K. Zumbrun, The Gap Lemma and a geometric condition for the instability of viscous shock profiles, Comm. Pure Appl. Math 51 (1998), no. 7, 797-855.

[14] A. Gierer and H. Meinhardt, A theory of biological pattern formation, Kybernetik 12 (1972), 30-39.

[15] D. Henry, Geometric theory of semilinear parabolic equations, Lecture Notes in Mathematics, vol. 840, Springer-Verlag, 1981.

[16] H. Ikeda, Y Nishiura, and H. Suzuki, Stability of traveling waves and a relation between the Evans function and the SLEP equation, J. Reine Angew. Math. 475 (1996), 1-37.

[17] D. Iron, M.J. Ward, and J. Wei, The stability of spike solutions to the one-dimensional Gierer-Meinhardt model, Physica D 150 (2001), no. 1-2, 25-62.

[18] C.K.R.T. Jones, Geometric singular perturbation theory, Dynamical Systems, Montecatibi Terme, 1994 (R. Johnson, ed.), Lecture Notes in Mathematics, vol. 1609, Springer-Verlag, 1994, pp. 44-118.

[19] T. Kapitula, The Evans function and generalized Melnikov integrals, SIAM J. Math. Anal. 30 (1999), no. 2, 273-297.

[20] T. Kapitula and B. Sandstede, Stability of bright and dark solitary wave solutions to perturbed nonlinear Schrodinger equations, Physica D 124 (1998), 58-103.

[21] T. Kapitula and B. Sandstede, Edge bifurcations for near integrable systems via Evans function techniques, SIAM J. Math. Anal. 33 (2002), no. 5, 1117-1143.

[22] G.L. Lamb, Elements of Soliton Theory, Wiley Interscience, 1980. 
[23] W. Magnus and S. Winkler, Hill's Equation, Wiley Interscience, 1966.

[24] W.-M. Ni, Diffusion, cross-diffusion, and their spike-layer steady states, Notices AMS 45 (1998), no. 1, 9-18.

[25] M. Oh and K. Zumbrun, Stability of periodic solutions of conservation laws with viscosity: Analysis of the Evans function, Arch. Rational Mech. Anal. 166 (2003), 99-166.

[26] R.L. Pego and M.I. Weinstein, Eigenvalues, and instabilities of solitary waves, Phil. Trans. Roy. Soc. London Ser. A 340 (1992), 47-94.

[27] B. Sandstede and A. Scheel, On the stability of periodic travelling waves with large spatial period, J. Diff. Eq. 172 (2001), 134-188.

[28] M.J. Ward and J. Wei, Asymmetric spike patterns for the one-dimensional GiererMeinhardt model: equilibria and stability, European J. Appl. Math. 13 (2002), no. 3, 283-320.

[29] M.J. Ward and J. Wei, Hopf bifurcations and oscillatory instabilities of spike solutions for the one-dimensional Gierer-Meinhardt model, J. Nonlinear Sci. 13 (2003), no. 2, 209-264. 